\title{
Effective Asymmetric Epoxidation of Styrenes by Chiral Dioxirane
}

David Goeddel, Lianhe Shu, Yi Yuan, O. Andrea Wong, Bin Wang, and Yian Shi*

Department of Chemistry

Colorado State University

Fort Collins, CO 80523

Tel: (970)-491-7424

Fax: (970)-491-1801

Email: yian@lamar.colostate.edu

\section{Supporting Information}

\section{Table of Contents}

Spectral Data of Ketones

S2-S17

The Chromatograms for Epoxide EE Determination

S18-S24

NMR Spectra

S25-S54 


\section{Spectral Data of Ketones 4}

\section{Ketone 4a}

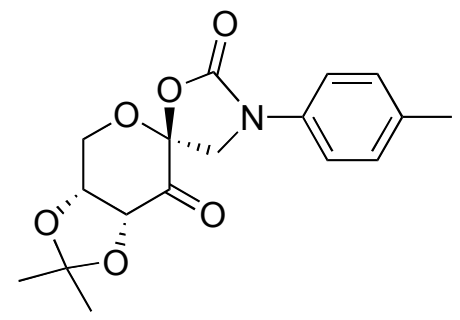

Shu, L.; Wang, P.; Gan, Y.; Shi, Y. Org. Lett. 2003, 5, 293

mp. 162.0-163.5 ${ }^{\circ} \mathrm{C}$; IR (film) $1774 \mathrm{~cm}^{-1} ;{ }^{1} \mathrm{H}$ NMR (300 MHz, $\left.\mathrm{CDCl}_{3}\right) \delta 7.39$ (m, 2H), 7.18 (m, 2H), $4.87(\mathrm{~d}, J=5.1 \mathrm{~Hz}, 1 \mathrm{H}), 4.73(\mathrm{~d}, J=10.8 \mathrm{~Hz}, 1 \mathrm{H}), 4.66-4.60(\mathrm{~m}, 2 \mathrm{H}), 4.26(\mathrm{dd}, J=$ 13.5, 0.6 Hz, 1H), $3.74(\mathrm{~d}, J=10.8 \mathrm{~Hz}, 1 \mathrm{H}), 2.33(\mathrm{~s}, 3 \mathrm{H}), 1.49(\mathrm{~s}, 3 \mathrm{H}), 1.43(\mathrm{~s}, 3 \mathrm{H}) ;{ }^{13} \mathrm{C} \mathrm{NMR}$ $\left(75 \mathrm{MHz}, \mathrm{CDCl}_{3}\right) \delta 195.1,151.3,134.9,134.6,129.9,118.9,111.2,99.2,77.6,75.7,61.1,50.0$, 27.4, 26.3, 21.1. HRMS calcd for $\mathrm{C}_{17} \mathrm{H}_{19} \mathrm{NO}_{6}\left(\mathrm{M}^{+}\right)$333.1212, found 333.1210.

\section{Ketone 4b}

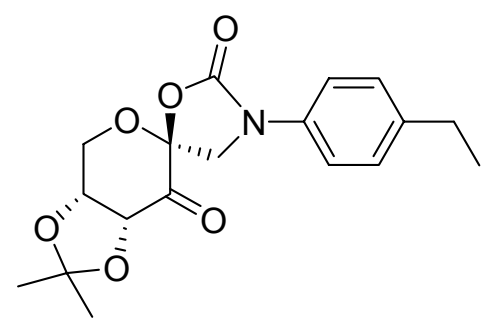

mp $135-136{ }^{\circ} \mathrm{C} ;[\alpha]^{20}=-40.6\left(c, 0.32, \mathrm{CHCl}_{3}\right) ; \mathrm{IR}\left(\right.$ film) $1774 \mathrm{~cm}^{-1} ;{ }^{1} \mathrm{H}$ NMR $(300 \mathrm{MHz}$, $\left.\mathrm{CDCl}_{3}\right) \delta 7.42(\mathrm{~d}, J=8.4 \mathrm{~Hz}, 2 \mathrm{H}), 7.21(\mathrm{~d}, J=8.4 \mathrm{~Hz}, 2 \mathrm{H}), 4.87(\mathrm{~d}, J=5.1 \mathrm{~Hz}, 1 \mathrm{H}), 4.74(\mathrm{~d}, J=$ $10.2 \mathrm{~Hz}, 1 \mathrm{H}), 4.66-4.59(\mathrm{~m}, 2 \mathrm{H}), 4.26(\mathrm{~d}, J=13.5 \mathrm{~Hz}, 1 \mathrm{H}), 3.75(\mathrm{~d}, J=10.2 \mathrm{~Hz}, 1 \mathrm{H}), 2.63(\mathrm{q}, J$

$=7.5 \mathrm{~Hz}, 2 \mathrm{H}), 1.48(\mathrm{~s}, 3 \mathrm{H}), 1.43(\mathrm{~s}, 3 \mathrm{H}), 1.22(\mathrm{t}, J=7.5 \mathrm{~Hz}, 3 \mathrm{H}) ;{ }^{13} \mathrm{C} \mathrm{NMR}\left(75 \mathrm{MHz}, \mathrm{CDCl}_{3}\right) \delta$ 
$195.1,151.4,141.3,134.7,128.7,119.0,111.2,99.3,77.7,75.7,61.2,50.1,28.5,27.4,26.3$, 15.9. HRMS calcd for $\mathrm{C}_{18} \mathrm{H}_{22} \mathrm{NO}_{6}\left(\mathrm{M}^{+}+1\right) 348.1447$, found 348.1441 .

\section{Ketone 4c}

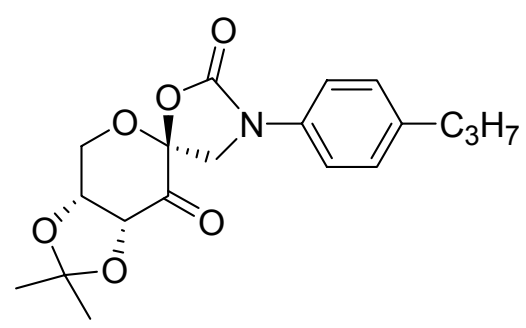

mp 135-136 ${ }^{\circ} \mathrm{C} ;[\alpha]^{20}{ }_{\mathrm{D}}=-28.4\left(c, 0.66, \mathrm{CHCl}_{3}\right) ; \quad$ IR (film) $1776 \mathrm{~cm}^{-1} ;{ }^{1} \mathrm{H}$ NMR $(300 \mathrm{MHz}$, $\left.\mathrm{CDCl}_{3}\right) \delta 7.41(\mathrm{~d}, J=8.4 \mathrm{~Hz}, 2 \mathrm{H}), 7.19(\mathrm{~d}, J=8.4 \mathrm{~Hz}, 2 \mathrm{H}), 4.86(\mathrm{~d}, J=5.4 \mathrm{~Hz}, 1 \mathrm{H}), 4.73(\mathrm{~d}, J$ $=10.2 \mathrm{~Hz}, 1 \mathrm{H}), 4.66-4.58(\mathrm{~m}, 2 \mathrm{H}), 4.25(\mathrm{~d}, J=14.4 \mathrm{~Hz}, 1 \mathrm{H}), 3.74(\mathrm{~d}, J=10.2 \mathrm{~Hz}, 1 \mathrm{H}), 2.56(\mathrm{t}, J$ $=7.5 \mathrm{~Hz}, 2 \mathrm{H}), 1.68-1.54(\mathrm{~m}, 2 \mathrm{H}), 1.48(\mathrm{~s}, 3 \mathrm{H}), 1.42(\mathrm{~s}, 3 \mathrm{H}), 0.92(\mathrm{t}, J=7.5 \mathrm{~Hz}, 3 \mathrm{H}) ;{ }^{13} \mathrm{C} \mathrm{NMR}$ $\left(75 \mathrm{MHz}, \mathrm{CDCl}_{3}\right) \delta 195.1,151.3,139.7,134.7,129.3,118.9,111.2,99.2,77.7,75.7,61.1,50.0$, 37.5, 27.4, 26.2, 24.8, 14.0. Anal. Calcd for $\mathrm{C}_{19} \mathrm{H}_{23} \mathrm{NO}_{6}$ : C, 63.15; H, 6.41; N, 3.88. Found: C, $62.88 ; \mathrm{H}, 6.41 ; \mathrm{N}, 3.61$.

\section{Ketone 4d}

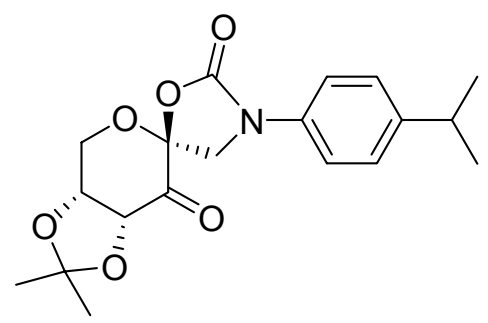

mp $180-182{ }^{\circ} \mathrm{C} ;[\alpha]^{20}{ }_{\mathrm{D}}=-33.1\left(c, 0.51, \mathrm{CHCl}_{3}\right) ; \quad$ IR (film) $1775 \mathrm{~cm}^{-1} ;{ }^{1} \mathrm{H}$ NMR $(300 \mathrm{MHz}$, $\left.\mathrm{CDCl}_{3}\right) \delta 7.43(\mathrm{~d}, J=8.4 \mathrm{~Hz}, 2 \mathrm{H}), 7.24(\mathrm{~d}, J=8.4 \mathrm{~Hz}, 2 \mathrm{H}), 4.87(\mathrm{~d}, J=5.1 \mathrm{~Hz}, 1 \mathrm{H}), 4.75(\mathrm{~d}, J=$ $10.5 \mathrm{~Hz}, 1 \mathrm{H}), 4.67-4.60(\mathrm{~m}, 2 \mathrm{H}), 4.26(\mathrm{~d}, J=14.4 \mathrm{~Hz}, 1 \mathrm{H}), 3.75(\mathrm{~d}, J=10.5 \mathrm{~Hz}, 1 \mathrm{H}), 2.96-2.84$ 
$(\mathrm{m}, 1 \mathrm{H}), 1.48(\mathrm{~s}, 3 \mathrm{H}), 1.43(\mathrm{~s}, 3 \mathrm{H}), 1.23(\mathrm{~d}, J=6.9 \mathrm{~Hz}, 6 \mathrm{H}) ;{ }^{13} \mathrm{C} \mathrm{NMR}\left(100 \mathrm{MHz}, \mathrm{CDCl}_{3}\right) \delta$ 195.3, 151.4, 146.0, 134.8, 127.3, 119.1, 111.2, 99.2, 77.6, 75.6, 61.1, 50.0, 33.7, 27.3, 26.1, 24.1. Anal. Calcd for $\mathrm{C}_{19} \mathrm{H}_{23} \mathrm{NO}_{6}: \mathrm{C}, 63.15 ; \mathrm{H}, 6.41 ; \mathrm{N}, 3.88$. Found: $\mathrm{C}, 63.24 ; \mathrm{H}, 6.39 ; \mathrm{N}, 3.76$.

\section{Ketone 4e}

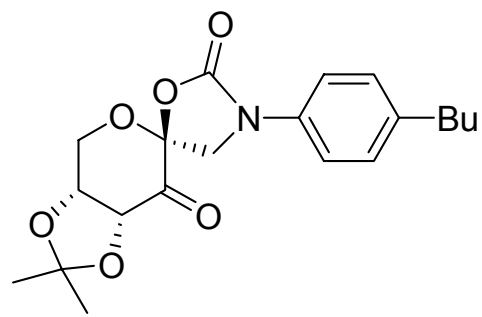

mp 164-166 ${ }^{\circ} \mathrm{C} ;[\alpha]^{20}{ }_{\mathrm{D}}=-32.9\left(c, 0.28, \mathrm{CHCl}_{3}\right) ; \quad$ IR (film) $1776 \mathrm{~cm}^{-1} ;{ }^{1} \mathrm{H}$ NMR $(300 \mathrm{MHz}$, $\left.\mathrm{CDCl}_{3}\right) \delta$ 7.45-7.39 (m, 2H), 7.22-7.14 (m, 2H), $4.87(\mathrm{~d}, J=5.4 \mathrm{~Hz}, 1 \mathrm{H}), 4.74(\mathrm{~d}, J=10.5 \mathrm{~Hz}$ 1H), 4.66-4.60 (m, 2H), $4.26(\mathrm{~d}, J=13.8 \mathrm{~Hz}, 1 \mathrm{H}), 3.75(\mathrm{~d}, J=10.5 \mathrm{~Hz}, 1 \mathrm{H}), 2.59(\mathrm{t}, J=7.5 \mathrm{~Hz}$, 2H), 1.63-1.57 (m, 2H), $1.48(\mathrm{~s}, 3 \mathrm{H}), 1.43(\mathrm{~s}, 3 \mathrm{H}), 1.41-1.26(\mathrm{~m}, 2 \mathrm{H}), 0.91(\mathrm{t}, J=7.5 \mathrm{~Hz}, 3 \mathrm{H})$; ${ }^{13} \mathrm{C}$ NMR $\left(75 \mathrm{MHz}, \mathrm{CDCl}_{3}\right) \delta 195.3,151.5,140.1,134.8,129.4,119.0,111.3,99.3,77.7,75.7$, 61.1, 50.0, 35.1, 33.8, 27.3, 26.2, 22.4, 14.1. Anal. Calcd for $\mathrm{C}_{20} \mathrm{H}_{25} \mathrm{NO}_{6}$ : C, 63.99; $\mathrm{H}, 6.71 ; \mathrm{N}$, 3.73. Found: C, 64.00; H, 6.77; N, 3.76 .

\section{Ketone 4f}

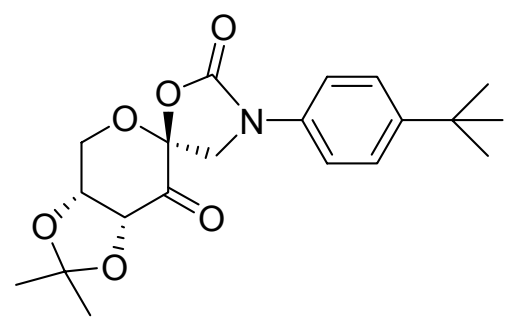

mp 172-174 ${ }^{\circ} \mathrm{C} ;[\alpha]^{20}{ }_{\mathrm{D}}=-36.0\left(c, 0.28, \mathrm{CHCl}_{3}\right) ;$ IR (film) $1775 \mathrm{~cm}^{-1} ;{ }^{1} \mathrm{H} \mathrm{NMR}(400 \mathrm{MHz}$, $\left.\mathrm{CDCl}_{3}\right) \delta$ 7.47-7.36 (m, 4H), $4.87(\mathrm{~d}, J=5.2 \mathrm{~Hz}, 1 \mathrm{H}), 4.75(\mathrm{~d}, J=10.4 \mathrm{~Hz}, 1 \mathrm{H}), 4.67-4.60(\mathrm{~m}$, 
2H), $4.27(\mathrm{~d}, J=14.0 \mathrm{~Hz}, 1 \mathrm{H}), 3.75$ (d, $J=10.4 \mathrm{~Hz}, 1 \mathrm{H}), 1.49$ (s, 3H), 1.43 (s, 3H), 1.31 (s, 9H);

${ }^{13} \mathrm{C}$ NMR $\left(100 \mathrm{MHz}, \mathrm{CDCl}_{3}\right) \delta 195.3,151.5,148.3,134.6,126.3,118.8,111.3,99.3,77.7,75.7$, 61.1, 50.0, 34.6, 31.5, 27.3, 26.2. HRMS calcd for $\mathrm{C}_{20} \mathrm{H}_{26} \mathrm{NO}_{6}\left(\mathrm{M}^{+}+1\right)$ 376.1760, found 376.1764 .

\section{Ketone 4g}

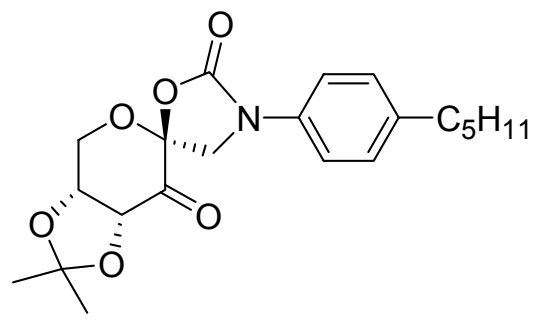

mp 93-94 ${ }^{\circ} \mathrm{C} ; \quad[\alpha]^{20}{ }_{\mathrm{D}}=-26.9\left(c, 0.36, \mathrm{CHCl}_{3}\right) ; \quad$ IR (film) $1776 \mathrm{~cm}^{-1} ;{ }^{1} \mathrm{H}$ NMR $(300 \mathrm{MHz}$, $\left.\mathrm{CDCl}_{3}\right) \delta 7.42(\mathrm{~d}, J=7.8 \mathrm{~Hz}, 2 \mathrm{H}), 7.20(\mathrm{~d}, \mathrm{~J}=7.8 \mathrm{~Hz}, 2 \mathrm{H}), 4.87(\mathrm{~d}, J=5.4 \mathrm{~Hz}, 1 \mathrm{H}), 4.75(\mathrm{~d}, J=$ $10.8 \mathrm{~Hz}, 1 \mathrm{H}), 4.67-4.60(\mathrm{~m}, 2 \mathrm{H}), 4.26(\mathrm{~d}, J=14.4 \mathrm{~Hz}, 1 \mathrm{H}), 3.75(\mathrm{~d}, J=10.8 \mathrm{~Hz}, 1 \mathrm{H}), 2.59(\mathrm{t}, J=$ $7.2 \mathrm{~Hz}, 2 \mathrm{H}), 1.66-1.52(\mathrm{~m}, 2 \mathrm{H}), 1.49(\mathrm{~s}, 3 \mathrm{H}), 1.44(\mathrm{~s}, 3 \mathrm{H}), 1.36-1.24(\mathrm{~m}, 4 \mathrm{H}), 0.89$ (t, $J=6.9 \mathrm{~Hz}$ $3 \mathrm{H}) ;{ }^{13} \mathrm{C}$ NMR $\left(75 \mathrm{MHz}, \mathrm{CDCl}_{3}\right) \delta 195.2,151.3,139.9,134.7,129.2,118.8,111.1,99.2,77.6$, 75.6, 61.0, 49.9, 35.3, 31.4, 31.2, 27.2, 26.1, 22.6, 14.1. Anal. Calcd for $\mathrm{C}_{21} \mathrm{H}_{27} \mathrm{NO}_{6}$ : C, 64.77; H, 6.99; N, 3.60. Found: C, 64.90; H, 6.99; N, 3.49.

\section{Ketone 4h}

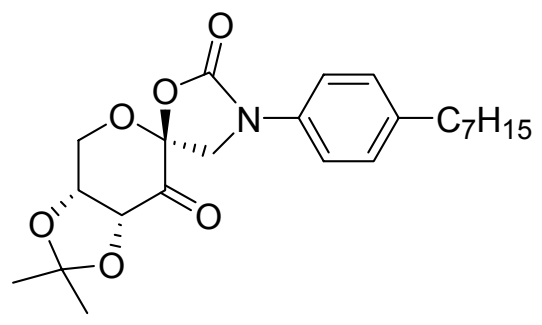


mp 71-72 ${ }^{\circ} \mathrm{C} ; \quad[\alpha]^{20}{ }_{\mathrm{D}}=-30.5\left(c, 0.44, \mathrm{CHCl}_{3}\right) ; \quad$ IR (film) $1777 \mathrm{~cm}^{-1} ;{ }^{1} \mathrm{H}$ NMR $(300 \mathrm{MHz}$, $\left.\mathrm{CDCl}_{3}\right) \delta 7.41(\mathrm{~d}, J=8.4 \mathrm{~Hz}, 2 \mathrm{H}), 7.19(\mathrm{~d}, J=8.4 \mathrm{~Hz}, 2 \mathrm{H}), 4.87(\mathrm{~d}, J=5.1 \mathrm{~Hz}, 1 \mathrm{H}), 4.74(\mathrm{~d}, J=$ $10.5 \mathrm{~Hz}, 1 \mathrm{H}), 4.67-4.60(\mathrm{~m}, 2 \mathrm{H}), 4.26(\mathrm{~d}, J=13.8 \mathrm{~Hz}, 1 \mathrm{H}), 3.75(\mathrm{~d}, J=10.5 \mathrm{~Hz}, 1 \mathrm{H}), 2.58(\mathrm{t}, J=$ $7.5 \mathrm{~Hz}, 2 \mathrm{H}), 1.66-1.52(\mathrm{~m}, 2 \mathrm{H}), 1.49(\mathrm{~s}, 3 \mathrm{H}), 1.43(\mathrm{~s}, 3 \mathrm{H}), 1.36-1.22(\mathrm{~m}, 8 \mathrm{H}), 0.88(\mathrm{t}, J=7.2 \mathrm{~Hz}$ $3 \mathrm{H}) ;{ }^{13} \mathrm{C}$ NMR $\left(75 \mathrm{MHz}, \mathrm{CDCl}_{3}\right) \delta 195.3,151.3,139.9,134.7,129.2,118.8,111.1,99.2,77.6$, 75.6, 61.0, 49.9, 35.3, 31.8, 31.5, 29.2, 27.2, 26.0, 22.7, 14.2. Anal. Calcd for $\mathrm{C}_{23} \mathrm{H}_{31} \mathrm{NO}_{6}$ : C, 66.17; H, 7.48; N, 3.35. Found: C, 66.10; H, 7.55; N, 3.18 .

\section{Ketone 4i}

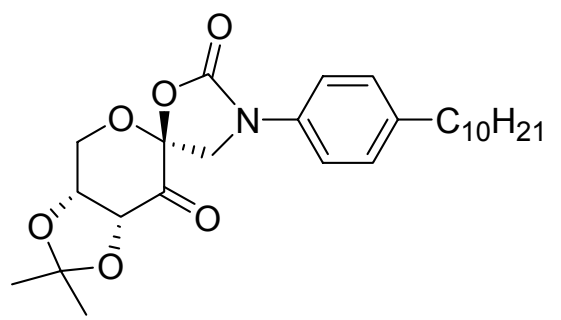

mp 110-111 ${ }^{\circ} \mathrm{C} ;[\alpha]^{20}{ }_{\mathrm{D}}=-40.6\left(c, 0.32, \mathrm{CHCl}_{3}\right)$; IR (film) 3436, $1774 \mathrm{~cm}^{-1}$; ketone: ${ }^{1} \mathrm{H}$ NMR $\left(300 \mathrm{MHz}, \mathrm{CDCl}_{3}\right) \delta$ 7.44-7.38 (m, 2H), 7.24-7.12 (m, 2H), $4.87(\mathrm{~d}, J=5.1 \mathrm{~Hz}, 1 \mathrm{H}), 4.74(\mathrm{~d}, J=$ $10.5 \mathrm{~Hz}, 1 \mathrm{H}), 4.68-4.60(\mathrm{~m}, 2 \mathrm{H}), 4.26(\mathrm{~d}, J=13.8 \mathrm{~Hz}, 1 \mathrm{H}), 3.75(\mathrm{~d}, J=10.5 \mathrm{~Hz}, 1 \mathrm{H}), 2.62-2.54$ (m, 2H), 1.63-1.52 (m, 2H), $1.49(\mathrm{~s}, 3 \mathrm{H}), 1.43(\mathrm{~s}, 3 \mathrm{H}), 1.34-1.20(\mathrm{~m}, 14 \mathrm{H}), 0.87(\mathrm{t}, J=6.9 \mathrm{~Hz}$ $3 \mathrm{H})$; hydrate: ${ }^{13} \mathrm{C} \mathrm{NMR}\left(100 \mathrm{MHz}, \mathrm{CDCl}_{3}\right) \delta 153.3,139.0,135.1,128.8,118.8,110.0,101.9$, $91.2,76.0,73.3,63.3,53.6,35.2,31.8,31.4,29.6,29.5,29.3,29.2,26.1,24.6,22.6,14.0$. Anal. Calcd for $\mathrm{C}_{26} \mathrm{H}_{37} \mathrm{NO}_{6}$ : C, 67.95, H, 8.11, N, 3.05. Found: C, 68.12, H, 7.94, N, 3.04. 


\section{Ketone 4j}

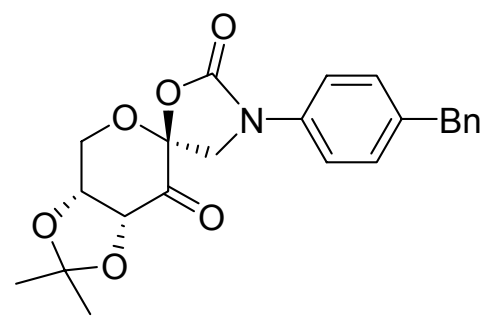

$\operatorname{mp~} 48-50{ }^{\circ} \mathrm{C} ;[\alpha]^{20}{ }_{\mathrm{D}}=-47.2\left(c, 0.13, \mathrm{CHCl}_{3}\right) ; \mathrm{IR}\left(\right.$ film) $3406,1762 \mathrm{~cm}^{-1} ;{ }^{1} \mathrm{H}$ NMR $(300 \mathrm{MHz}$, $\left.\mathrm{CDCl}_{3}\right) \delta$ 7.46-7.38 (m, 2H), 7.31-7.13 (m, 7H), $4.86(\mathrm{~d}, J=5.6 \mathrm{~Hz}, 1 \mathrm{H}), 4.72(\mathrm{~d}, J=10.4 \mathrm{~Hz}$ 1H), 4.65-4.58 (m, 2H), $4.25(\mathrm{~d}, J=12.8 \mathrm{~Hz}, 1 \mathrm{H}), 3.96(\mathrm{~s}, 2 \mathrm{H}), 3.73(\mathrm{~d}, J=10.4 \mathrm{~Hz}, 1 \mathrm{H}), 1.48$ (s, 3H), $1.42(\mathrm{~s}, 3 \mathrm{H}) ;{ }^{13} \mathrm{C}$ NMR (100 MHz, $\left.\mathrm{CDCl}_{3}\right)$ 195.3, 151.4, 140.9, 138.2, 135.3, 129.9, 129.0, 128.7, 126.4, 119.0, 111.2, 99.3, 77.6, 75.6, 61.1, 49.9, 41.4, 27.3, 26.1. HRMS calcd for $\mathrm{C}_{23} \mathrm{H}_{24} \mathrm{NO}_{6}\left(\mathrm{M}^{+}+1\right)$ 410.1604, found 410.1603 .

\section{Ketone 4k}

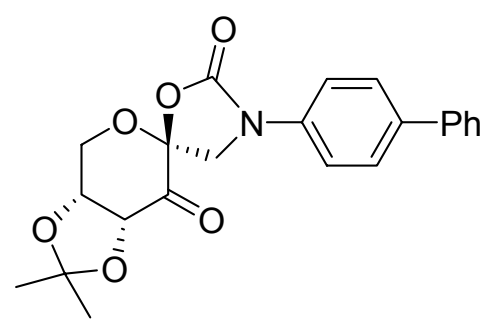

mp 76-78 ${ }^{\circ} \mathrm{C} ; \quad[\alpha]^{20}{ }_{\mathrm{D}}=-77.9\left(c, 0.20, \mathrm{CHCl}_{3}\right) ; \quad$ IR (film) $1776 \mathrm{~cm}^{-1} ;{ }^{1} \mathrm{H}$ NMR $(400 \mathrm{MHz}$, $\left.\mathrm{CDCl}_{3}\right) \delta$ 7.65-7.55 (m, 6H), 7.46-7.42 (m, 2H), 7.37-7.33 (m, 1H), $4.89(\mathrm{~d}, J=5.6 \mathrm{~Hz}, 1 \mathrm{H})$, $4.80(\mathrm{~d}, J=10.4 \mathrm{~Hz}, 1 \mathrm{H}), 4.68-4.61(\mathrm{~m}, 2 \mathrm{H}), 4.28(\mathrm{~d}, J=12.8 \mathrm{~Hz}, 1 \mathrm{H}), 3.81(\mathrm{~d}, J=10.4 \mathrm{~Hz}$, 1H), $1.50(\mathrm{~s}, 3 \mathrm{H}), 1.44(\mathrm{~s}, 3 \mathrm{H}) ;{ }^{13} \mathrm{C} \mathrm{NMR}\left(100 \mathrm{MHz}, \mathrm{CDCl}_{3}\right) \delta 195.2,151.3,140.2,138.0$, 136.4, 129.0, 128.0, 127.6, 127.1, 119.1, 111.3, 99.3, 77.7, 75.7, 61.2, 49.9, 27.3, 26.2; HRMS calcd for $\mathrm{C}_{22} \mathrm{H}_{22} \mathrm{NO}_{6}\left(\mathrm{M}^{+}+1\right)$ 396.1447, found 396.1440. 


\section{Ketone 41}

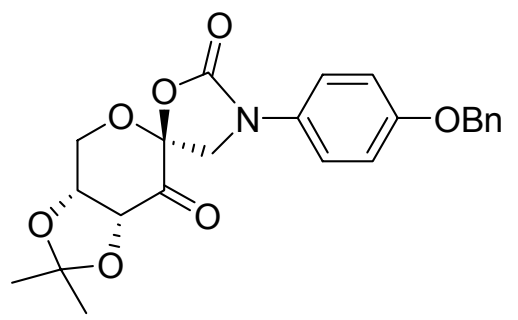

mp 52-56 ${ }^{\circ} \mathrm{C} ; \quad[\alpha]^{20}{ }_{\mathrm{D}}=-39.2\left(c, 0.28, \mathrm{CHCl}_{3}\right) ; \quad$ IR (film) $1764 \mathrm{~cm}^{-1} ;{ }^{1} \mathrm{H}$ NMR $(300 \mathrm{MHz}$, $\left.\mathrm{CDCl}_{3}\right) \delta 7.43-7.26(\mathrm{~m}, 7 \mathrm{H}), 7.01-6.92(\mathrm{~m}, 2 \mathrm{H}), 5.05(\mathrm{~s}, 2 \mathrm{H}), 4.86(\mathrm{~d}, J=4.8 \mathrm{~Hz}, 1 \mathrm{H}), 4.71(\mathrm{~d}, J$ $=10.8 \mathrm{~Hz}, 1 \mathrm{H}), 4.66-4.58(\mathrm{~m}, 2 \mathrm{H}), 4.26(\mathrm{~d}, J=14.4 \mathrm{~Hz}, 1 \mathrm{H}), 3.72(\mathrm{~d}, J=10.8 \mathrm{~Hz}, 1 \mathrm{H}), 1.48(\mathrm{~s}$, 3H), 1.43 (s, $3 \mathrm{H}) ;{ }^{13} \mathrm{C}$ NMR (100 MHz, $\left.\mathrm{CDCl}_{3}\right)$ 195.3, 156.3, 151.6, 136.9, 130.4, 128.8, 128.3, 127.6, 121.0, 115.7, 111.2, 99.3, 77.7, 75.7, 70.5, 61.1, 50.3, 27.3, 26.2; HRMS calcd for $\mathrm{C}_{23} \mathrm{H}_{23} \mathrm{NO}_{7}\left(\mathrm{M}^{+}\right)$425.1475, found 425.1469 .

\section{Ketone 4m}

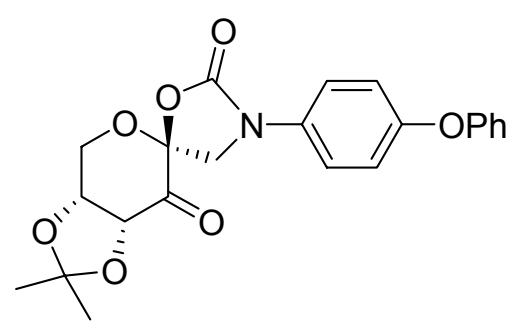

mp $136-138{ }^{\circ} \mathrm{C} ;[\alpha]^{20}{ }_{\mathrm{D}}=-5.9\left(c, 1.75, \mathrm{CHCl}_{3}\right)$; IR (film) $3385,1752 \mathrm{~cm}^{-1}$; hydrate: ${ }^{1} \mathrm{H}$ NMR $\left(400 \mathrm{MHz}, \mathrm{CDCl}_{3}\right) \delta$ 7.46-7.40 (m, 2H), 7.35-7.26 (m, 2H), 7.12-7.04 (m, 1H), 7.00-6.94 (m, 4H), $4.59(\mathrm{~d}, J=10.0 \mathrm{~Hz}, 1 \mathrm{H}), 4.46(\mathrm{~d}, J=7.2 \mathrm{~Hz}, 1 \mathrm{H}), 4.42(\mathrm{~m}, 1 \mathrm{H}), 4.33(\mathrm{~d}, J=12.8,2.4 \mathrm{~Hz}$ 1H), 3.95 (d, $J=12.8 \mathrm{~Hz}, 1 \mathrm{H}), 3.80$ (d, $J=10.0 \mathrm{~Hz}, 1 \mathrm{H}), 2.32$ (brs, H), 1.56 (s, 3H), 1.38 (S, $3 \mathrm{H}) ;{ }^{13} \mathrm{C} \mathrm{NMR}\left(100 \mathrm{MHz}, \mathrm{CDCl}_{3}\right) \delta 157.4,154.1,153.5,132.9,129.9,123.5,120.9,119.6$, 
118.8, 110.4, 102.0, 91.2, 76.2, 73.4, 63.4, 53.8, 26.4, 24.8. HRMS calcd for $\mathrm{C}_{22} \mathrm{H}_{21} \mathrm{NO}_{7}\left(\mathrm{M}^{+}\right)$ 411.1318, found 411.1326 .

\section{Ketone 4n}

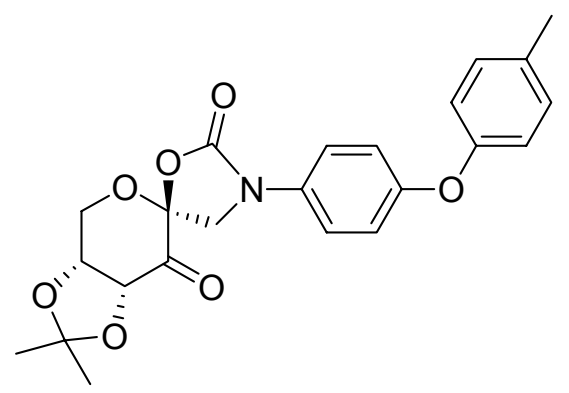

mp 56-59 ${ }^{\circ} \mathrm{C} ;[\alpha]^{20}{ }_{\mathrm{D}}=-47.6\left(c, 0.15, \mathrm{CHCl}_{3}\right) ; \mathrm{IR}\left(\right.$ film) $3419,1760 \mathrm{~cm}^{-1} ;{ }^{1} \mathrm{H} \mathrm{NMR}(300 \mathrm{MHz}$, $\left.\mathrm{CDCl}_{3}\right) \delta$ 7.48-7.40 (m, 2H), 7.16-7.08 (m, 2H), 7.02-6.85 (m, 4H), $4.87(\mathrm{~d}, J=5.6 \mathrm{~Hz}, 1 \mathrm{H})$ $4.74(\mathrm{~d}, J=10.4, \mathrm{~Hz}, 1 \mathrm{H}), 4.66-4.59(\mathrm{~m}, 2 \mathrm{H}), 4.27(\mathrm{~d}, J=13.2 \mathrm{~Hz}, 1 \mathrm{H}), 3.74(\mathrm{~d}, J=10.4 \mathrm{~Hz}$ 1H), $2.33(\mathrm{~s}, 3 \mathrm{H}), 1.48(\mathrm{~s}, 3 \mathrm{H}), 1.43(\mathrm{~s}, 3 \mathrm{H}) ;{ }^{13} \mathrm{C} \mathrm{NMR}\left(100 \mathrm{MHz}, \mathrm{CDCl}_{3}\right)$ 195.3, 155.2, 154.7, $151.6,133.3,132.1,130.5,120.8,119.2,119.1,111.2,99.3,77.6,75.6,61.1,50.2,27.3,26.1$, 20.9. HRMS calcd for $\mathrm{C}_{23} \mathrm{H}_{23} \mathrm{NO}_{7}\left(\mathrm{M}^{+}\right) 425.1475$, found 425.1465 .

\section{Ketone 4o}

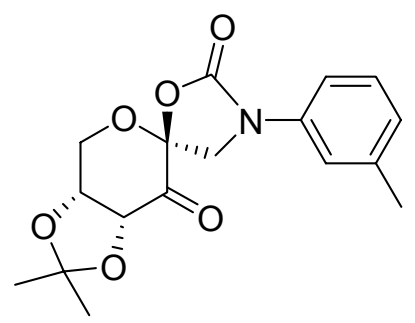

mp 116-118 ${ }^{\circ} \mathrm{C} ;[\alpha]^{20}{ }_{\mathrm{D}}=-24.3\left(c, 0.61, \mathrm{CHCl}_{3}\right) ; \quad$ IR (film) $1778,1760 \mathrm{~cm}^{-1} ;{ }^{1} \mathrm{H}$ NMR $(400$ $\left.\mathrm{MHz}, \mathrm{CDCl}_{3}\right) \delta 7.36-7.25(\mathrm{~m}, 3 \mathrm{H}), 6.99(\mathrm{~d}, J=6.8 \mathrm{~Hz}, 1 \mathrm{H}), 4.87(\mathrm{~d}, J=4.8 \mathrm{~Hz}, 1 \mathrm{H}), 4.74(\mathrm{~d}, J$ $=10.4 \mathrm{~Hz}, 1 \mathrm{H}), 4.67-4.59(\mathrm{~m}, 2 \mathrm{H}), 4.26(\mathrm{~d}, J=13.6 \mathrm{~Hz}, 1 \mathrm{H}), 3.75(\mathrm{~d}, J=10.4 \mathrm{~Hz}, 1 \mathrm{H}), 2.37(\mathrm{~s}$, 3H), $1.48(\mathrm{~s}, 3 \mathrm{H}), 1.43(\mathrm{~s}, 3 \mathrm{H}) ;{ }^{13} \mathrm{C} \mathrm{NMR}\left(100 \mathrm{MHz}, \mathrm{CDCl}_{3}\right) \delta$ 195.2, 151.2, 139.4, 137.1, 
129.2, 125.9, 119.5, 115.9, 111.1, 99.2, 77.6, 75.6, 61.0, 49.9, 27.2, 26.1, 21.7; Anal. Calcd for $\mathrm{C}_{17} \mathrm{H}_{19} \mathrm{NO}_{6}:$ C, 61.25; H, 5.75; N, 4.20. Found: $\mathrm{C}, 61.02 ; \mathrm{H}, 5.61 ; \mathrm{N}, 4.10$.

\section{Ketone 4p}

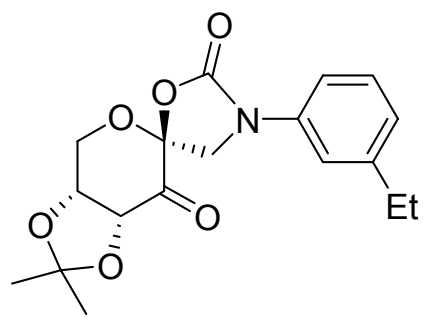

mp $135-136{ }^{\circ} \mathrm{C} ;[\alpha]^{20}{ }_{\mathrm{D}}=-25.1\left(c, 0.48, \mathrm{CHCl}_{3}\right)$; IR (film) $1777 \mathrm{~cm}^{-1} ;{ }^{1} \mathrm{H}$ NMR $(300 \mathrm{MHz}$, $\left.\mathrm{CDCl}_{3}\right) \delta 7.38-7.24(\mathrm{~m}, 3 \mathrm{H}), 7.03(\mathrm{~m}, 1 \mathrm{H}), 4.87(\mathrm{~d}, J=5.4 \mathrm{~Hz}, 1 \mathrm{H}), 4.77(\mathrm{~d}, J=10.5 \mathrm{~Hz}, 1 \mathrm{H})$, 4.67-4.60 (m, 2H), $4.27(\mathrm{~d}, J=14.4 \mathrm{~Hz}, 1 \mathrm{H}), 3.77(\mathrm{~d}, J=10.5 \mathrm{~Hz}, 1 \mathrm{H}), 2.67(\mathrm{q}, J=7.8 \mathrm{~Hz}, 2 \mathrm{H})$, 1.49 (s, 3H), 1.44 (s, 3H), 1.24 (t, $J=7.8 \mathrm{~Hz}, 3 \mathrm{H}) ;{ }^{13} \mathrm{C} \mathrm{NMR}\left(100 \mathrm{MHz}, \mathrm{CDCl}_{3}\right)$ 195.3, 151.4, $145.8,137.2,129.3,124.8,118.5,116.3,111.2,99.2,77.7,75.7,61.1,50.0,29.2,27.3,26.2$ 15.7. Anal. Calcd for $\mathrm{C}_{18} \mathrm{H}_{21} \mathrm{NO}_{6}$ : C, 62.24; $\mathrm{H}, 6.09 ; \mathrm{N}, 4.03$. Found: $\mathrm{C}, 62.50 ; \mathrm{H}, 6.13 ; \mathrm{N}$, 3.86 .

\section{Ketone 4q}

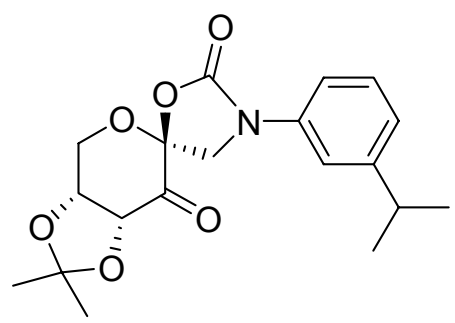

mp $125-126{ }^{\circ} \mathrm{C} ;[\alpha]^{20}{ }_{\mathrm{D}}=-26.5\left(c, 0.22, \mathrm{CHCl}_{3}\right) ; \mathrm{IR}\left(\right.$ film) $1775 \mathrm{~cm}^{-1} ;{ }^{1} \mathrm{H}$ NMR $(300 \mathrm{MHz}$, $\left.\mathrm{CDCl}_{3}\right) \delta 7.39(\mathrm{~s}, 1 \mathrm{H}), 7.33-7.30(\mathrm{~m}, 2 \mathrm{H}), 7.08-7.03(\mathrm{~m}, 2 \mathrm{H}), 4.87(\mathrm{~d}, J=5.1 \mathrm{~Hz}, 1 \mathrm{H}), 4.77(\mathrm{~d}, J$ $=10.5 \mathrm{~Hz}, 1 \mathrm{H}), 4.67-4.60(\mathrm{~m}, 2 \mathrm{H}), 4.27(\mathrm{~d}, J=14.1 \mathrm{~Hz}, 1 \mathrm{H}), 3.77(\mathrm{~d}, J=10.5 \mathrm{~Hz}, 1 \mathrm{H}), 3.00-$ $2.84(\mathrm{~m}, 1 \mathrm{H}), 1.49(\mathrm{~s}, 3 \mathrm{H}), 1.43(\mathrm{~s}, 3 \mathrm{H}), 1.25(\mathrm{~d}, J=6.9 \mathrm{~Hz}, 6 \mathrm{H}) ;{ }^{13} \mathrm{C} \mathrm{NMR}\left(100 \mathrm{MHz}, \mathrm{CDCl}_{3}\right) \delta$ 
195.3, 151.3, 150.4, 137.1, 129.3, 123.2, 117.1, 116.4, 111.2, 99.2, 77.6, 75.6, 61.0, 50.0, 34.4, 27.2, 26.1, 24.1, 24.0. Anal. Calcd for $\mathrm{C}_{19} \mathrm{H}_{23} \mathrm{NO}_{6}$ : C, 63.15; H, 6.41; N, 3.88. Found: C, $63.31 ; \mathrm{H}, 6.48 ; \mathrm{N}, 3.76$.

\section{Ketone 4r}

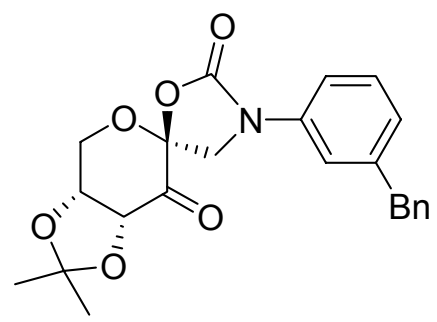

mp 58-60 ${ }^{\circ} \mathrm{C} ;[\alpha]^{20}{ }_{\mathrm{D}}=-46.0\left(c, 0.15, \mathrm{CHCl}_{3}\right)$; IR (film) $3411,1759 \mathrm{~cm}^{-1}$; ketone: ${ }^{1} \mathrm{H}$ NMR $\left(400 \mathrm{MHz}, \mathrm{CDCl}_{3}\right) \delta 7.38-7.16(\mathrm{~m}, 8 \mathrm{H}), 7.01(\mathrm{~d}, J=7.6 \mathrm{~Hz}, 1 \mathrm{H}), 4.86(\mathrm{~d}, J=5.6 \mathrm{~Hz}, 1 \mathrm{H}), 4.71$ (d, $J=10.4 \mathrm{~Hz}, 1 \mathrm{H}), 4.65-4.58(\mathrm{~m}, 2 \mathrm{H}), 4.24(\mathrm{~d}, J=13.6 \mathrm{~Hz}, 1 \mathrm{H}), 3.99(\mathrm{~s}, 2 \mathrm{H}), 3.72(\mathrm{~d}, J=10.4$ $\mathrm{Hz}, 1 \mathrm{H}), 1.48$ (s, 3H), 1.42 (s, 3H); hydrate: ${ }^{13} \mathrm{C} \mathrm{NMR}\left(100 \mathrm{MHz}, \mathrm{CDCl}_{3}\right)$ 153.1, 142.4, 140.7, $137.8,129.3,129.0,128.7,126.4,125.3,119.3,116.6,110.3,101.6,91.0,76.2,73.3,63.0,53.2$ 42.1, 27.3, 24.8; HRMS calcd for $\mathrm{C}_{23} \mathrm{H}_{24} \mathrm{NO}_{6}\left(\mathrm{M}^{+}+1\right) 410.1604$, found 410.1616 .

\section{Ketone 4s}

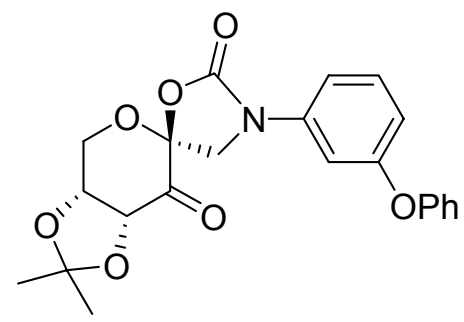

mp $136-138{ }^{\circ} \mathrm{C} ;[\alpha]^{20}{ }_{\mathrm{D}}=-40.8\left(c, 5.4, \mathrm{CHCl}_{3}\right) ;$ IR (film) $1778 \mathrm{~cm}^{-1} ;{ }^{1} \mathrm{H}$ NMR $(300 \mathrm{MHz}$, $\left.\mathrm{CDCl}_{3}\right) \delta$ 7.38-7.22 (m, 5H), 7.14-7.09 (m, 1H), 7.04-6.98 (m, 2H), 6.81-6.76 (m, 1H), $4.85(\mathrm{~d}$, $J=5.4 \mathrm{~Hz}, 1 \mathrm{H}), 4.70(\mathrm{~d}, J=10.5 \mathrm{~Hz}, 1 \mathrm{H}), 4.65-4.56(\mathrm{~m}, 2 \mathrm{H}), 4.25(\mathrm{~d}, J=12.6 \mathrm{~Hz}, 1 \mathrm{H}), 3.73(\mathrm{~d}$, 
$J=10.5 \mathrm{~Hz}, 1 \mathrm{H}), 1.47(\mathrm{~s}, 3 \mathrm{H}), 1.42(\mathrm{~s}, 3 \mathrm{H}) ;{ }^{13} \mathrm{C} \mathrm{NMR}\left(75 \mathrm{MHz}, \mathrm{CDCl}_{3}\right) \delta 195.0,158.1,156.6$ 151.0, 138.5, 130.3, 129.9, 123.8, 119.1, 114.9, 113.1, 111.2, 109.4, 99.2, 77.7, 75.6, 61.2, 49.9, 27.3, 26.2. HRMS calcd for $\mathrm{C}_{22} \mathrm{H}_{21} \mathrm{NO}_{7}\left(\mathrm{M}^{+}\right)$411.1318, found 411.1305.

\section{Ketone 4t}

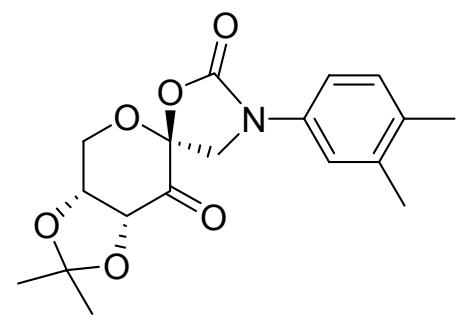

mp $191-193{ }^{\circ} \mathrm{C} ;[\alpha]^{20}{ }_{\mathrm{D}}=-37.4\left(c, 0.50, \mathrm{CHCl}_{3}\right) ; \mathrm{IR}$ (film) $1773,1756 \mathrm{~cm}^{-1} ;{ }^{1} \mathrm{H}$ NMR $(300$ $\left.\mathrm{MHz}, \mathrm{CDCl}_{3}\right) \delta 7.30-7.12(\mathrm{~m}, 3 \mathrm{H}), 4.87(\mathrm{~d}, J=5.1 \mathrm{~Hz}, 1 \mathrm{H}), 4.74(\mathrm{~d}, J=10.5 \mathrm{~Hz}, 1 \mathrm{H}), 4.67-4.60$ (m, 2H), $4.26(\mathrm{~d}, J=13.5 \mathrm{~Hz}, 1 \mathrm{H}), 3.73(\mathrm{~d}, J=10.5 \mathrm{~Hz}, 1 \mathrm{H}), 2.27$ (s, 3H), $2.24(\mathrm{~s}, 3 \mathrm{H}), 1.48(\mathrm{~s}$, 3H), $1.43(\mathrm{~s}, 3 \mathrm{H}) ;{ }^{13} \mathrm{C} \mathrm{NMR}\left(100 \mathrm{MHz}, \mathrm{CDCl}_{3}\right) \delta 195.3,151.4,137.7,134.8,133.6,130.3$, 120.3, 116.5, 111.1, 99.2, 77.6, 75.6, 61.0, 50.0, 27.2, 26.1, 20.1, 19.3. Anal. Calcd for $\mathrm{C}_{18} \mathrm{H}_{21} \mathrm{NO}_{6}: \mathrm{C}, 62.24 ; \mathrm{H}, 6.09 ; \mathrm{N}, 4.03$. Found: $\mathrm{C}, 62.30 ; \mathrm{H}, 5.85 ; \mathrm{N}, 3.99$.

\section{Ketone 4u}

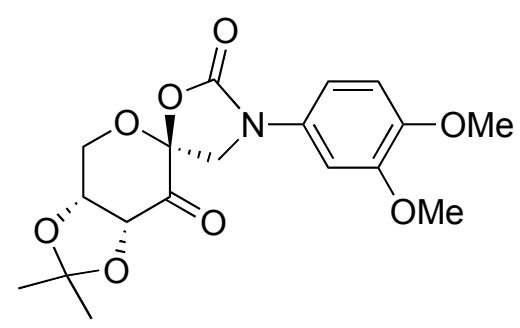

mp $108-110{ }^{\circ} \mathrm{C} ;[\alpha]^{20}{ }_{\mathrm{D}}=-21.2\left(c, 0.34, \mathrm{CHCl}_{3}\right) ; \quad$ IR (film) $1772 \mathrm{~cm}^{-1} ;{ }^{1} \mathrm{H}$ NMR $(400 \mathrm{MHz}$, $\left.\mathrm{CDCl}_{3}\right) \delta 7.35(\mathrm{~d}, J=2.2 \mathrm{~Hz}, 1 \mathrm{H}), 6.86(\mathrm{~d}, J=8.8 \mathrm{~Hz}, 1 \mathrm{H}), 6.79(\mathrm{dd}, J=8.8,2.2 \mathrm{~Hz}, 1 \mathrm{H}), 4.88$ (d, $J=5.2 \mathrm{~Hz}, 1 \mathrm{H}), 4.74(\mathrm{~d}, J=10.4 \mathrm{~Hz}, 1 \mathrm{H}), 4.66-4.60(\mathrm{~m}, 2 \mathrm{H}), 4.27(\mathrm{~d}, J=13.2 \mathrm{~Hz}, 1 \mathrm{H}), 3.91$ 
(s, 3H), 3.88 (s, 3H), 3.74 (d, $J=10.4 \mathrm{~Hz}, 1 \mathrm{H}), 1.48$ (s, 3H), 1.43 (s, 3H); ${ }^{13} \mathrm{C}$ NMR (100 MHz, $\left.\mathrm{CDCl}_{3}\right) \delta 195.3,151.4,149.4,146.6,130.6,111.3,111.1,111.0,104.0,99.1,77.6,75.6,61.0$ 56.2, 56.1, 50.3, 27.2, 26.1; HRMS calcd for $\mathrm{C}_{18} \mathrm{H}_{21} \mathrm{NO}_{8}\left(\mathrm{M}^{+}\right)$379.1267, found 379.1268.

\section{Ketone 4v}

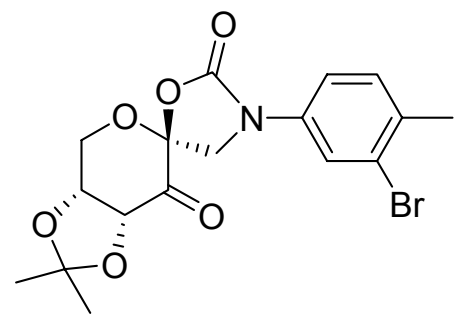

mp 146-148 ${ }^{\circ} \mathrm{C} ;[\alpha]^{20}{ }_{\mathrm{D}}=-44.4\left(c, 0.135, \mathrm{CHCl}_{3}\right)$; IR (film) 3404, $1774 \mathrm{~cm}^{-1}$; ketone: ${ }^{1} \mathrm{H}$ NMR $\left(300 \mathrm{MHz}, \mathrm{CDCl}_{3}\right) \delta 7.70(\mathrm{~d}, J=2.4 \mathrm{~Hz}, 1 \mathrm{H}), 7.37(\mathrm{dd}, J=8.4,2.4 \mathrm{~Hz}, 1 \mathrm{H}), 7.21(\mathrm{~d}, J=8.4 \mathrm{~Hz}$, $1 \mathrm{H}), 4.85(\mathrm{~d}, J=5.4 \mathrm{~Hz}, 1 \mathrm{H}), 4.69(\mathrm{~d}, J=10.2 \mathrm{~Hz}, 1 \mathrm{H}), 4.66-4.55(\mathrm{~m}, 2 \mathrm{H}), 4.25(\mathrm{~d}, J=12.6 \mathrm{~Hz}$, $1 \mathrm{H}), 3.71(\mathrm{~d}, J=10.2 \mathrm{~Hz}, 1 \mathrm{H}), 2.35(\mathrm{~s}, 3 \mathrm{H}), 1.46(\mathrm{~s}, 3 \mathrm{H}), 1.41(\mathrm{~s}, 3 \mathrm{H})$; hydrate: ${ }^{13} \mathrm{C}$ NMR $(100$ $\left.\mathrm{MHz}, \mathrm{CDCl}_{3}\right) \delta 152.9,136.4,133.7,130.7,124.9,122.1,117.4,110.1,101.9,91.1,76.0,73.3$ 63.4, 53.3, 26.2, 24.7, 22.1. HRMS calcd for $\mathrm{C}_{17} \mathrm{H}_{18} \mathrm{BrNO}_{6}\left(\mathrm{M}^{+} \mathrm{H}\right)$ 412.0396, found 412.0395.

\section{Ketone 4w}

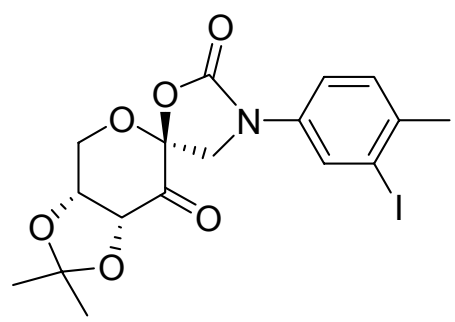

mp 161-162 ${ }^{\circ} \mathrm{C} ;[\alpha]^{20}{ }_{\mathrm{D}}=-17.8\left(c, 0.47, \mathrm{CHCl}_{3}\right) ; \quad$ IR (film) $1774 \mathrm{~cm}^{-1} ;{ }^{1} \mathrm{H}$ NMR $(300 \mathrm{MHz}$, $\left.\mathrm{CDCl}_{3}\right) \delta 7.92(\mathrm{~d}, J=2.4 \mathrm{~Hz}, 1 \mathrm{H}), 7.46(\mathrm{dd}, J=8.4,2.4 \mathrm{~Hz}, 1 \mathrm{H}), 7.23,(\mathrm{~d}, J=8.4 \mathrm{~Hz}, 1 \mathrm{H}), 4.86$ (d, $J=5.7 \mathrm{~Hz}, 1 \mathrm{H}), 4.71(\mathrm{~d}, J=10.2 \mathrm{~Hz}, 1 \mathrm{H}), 4.66-4.58(\mathrm{~m}, 2 \mathrm{H}), 4.27(\mathrm{~d}, J=13.8 \mathrm{~Hz}, 1 \mathrm{H}), 3.72$ 
$(\mathrm{d}, J=10.2 \mathrm{~Hz}, 1 \mathrm{H}), 2.41(\mathrm{~s}, 3 \mathrm{H}), 1.48(\mathrm{~s}, 3 \mathrm{H}), 1.43(\mathrm{~s}, 3 \mathrm{H}) ;{ }^{13} \mathrm{C} \mathrm{NMR}\left(75 \mathrm{MHz}, \mathrm{CDCl}_{3}\right) \delta$ 195.1, 151.1, 138.3, 135.7, 129.8, 128.7, 118.6, 111.2, 101.1, 99.3, 77.5, 75.6, 61.1, 49.8, 27.5, 27.2, 26.1. Anal. Calcd for $\mathrm{C}_{17} \mathrm{H}_{18} \mathrm{INO}_{6}$ : C, 44.46; H, 3.95; N, 3.05. Found: C, 44.26; H, 4.15; N, 2.76 .

\section{Ketone 4x}

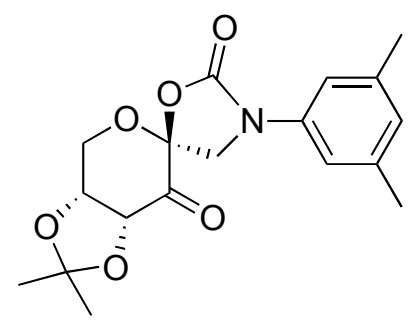

mp 154-155 ${ }^{\circ} \mathrm{C} ;[\alpha]^{20}{ }_{\mathrm{D}}=-23.5\left(c, 0.77, \mathrm{CHCl}_{3}\right) ;$ IR (film) $1776 \mathrm{~cm}^{-1} ;{ }^{1} \mathrm{H} \mathrm{NMR}(400 \mathrm{MHz}$, $\left.\mathrm{CDCl}_{3}\right) \delta 7.15(\mathrm{~s}, 2 \mathrm{H}), 6.84(\mathrm{~s}, 1 \mathrm{H}), 4.88(\mathrm{~d}, J=5.2 \mathrm{~Hz}, 1 \mathrm{H}), 4.76(\mathrm{~d}, J=10.8 \mathrm{~Hz}, 1 \mathrm{H}), 4.67-$ $4.63(\mathrm{~m}, 2 \mathrm{H}), 4.27(\mathrm{~d}, J=13.2 \mathrm{~Hz}, 1 \mathrm{H}), 3.75(\mathrm{~d}, J=10.8 \mathrm{~Hz}, 1 \mathrm{H}), 2.34(\mathrm{~s}, 6 \mathrm{H}), 1.50(\mathrm{~s}, 3 \mathrm{H})$, $1.45(\mathrm{~s}, 3 \mathrm{H}) ;{ }^{13} \mathrm{C} \mathrm{NMR}\left(100 \mathrm{MHz}, \mathrm{CDCl}_{3}\right) \delta 195.3,139.3,137.1,127.0,116.9,111.3,99.2$, 77.7, 75.7, 61.1, 50.1, 27.3, 26.2, 21.7. HRMS calcd for $\mathrm{C}_{18} \mathrm{H}_{21} \mathrm{NO}_{6}\left(\mathrm{M}^{+}\right)$347.1369, found 347.1370.

\section{Ketone 4y}<smiles>COc1cc(N2C[C@]3(OC[C@H]4OC(C)(C)O[C@H]4C3=O)OC2=O)cc(OC)c1OC</smiles> 
mp 58-66 ${ }^{\circ} \mathrm{C} ;[\alpha]^{20}{ }_{\mathrm{D}}=-44.8\left(c, 0.25, \mathrm{CHCl}_{3}\right)$; IR (film) $3450,1759 \mathrm{~cm}^{-1}$; hydrate: ${ }^{1} \mathrm{H}$ NMR $\left(400 \mathrm{MHz}, \mathrm{CDCl}_{3}\right) \delta 6.75(\mathrm{~s}, 2 \mathrm{H}), 4.62(\mathrm{~d}, J=10.0 \mathrm{~Hz}, 1 \mathrm{H}), 4.47(\mathrm{~d}, J=6.8 \mathrm{~Hz}, 1 \mathrm{H}), 4.42(\mathrm{~m}$, 1H), $4.32(\mathrm{dd}, J=12.8,2.4 \mathrm{~Hz}, 1 \mathrm{H}), 3.94(\mathrm{~d}, J=12.8 \mathrm{~Hz}, 1 \mathrm{H}), 3.82-3.78(\mathrm{~m}, 10 \mathrm{H}), 1.56(\mathrm{~s}, 3 \mathrm{H})$, $1.39(\mathrm{~s}, 3 \mathrm{H}) ;{ }^{13} \mathrm{C}$ NMR $\left(100 \mathrm{MHz}, \mathrm{CDCl}_{3}\right) \delta 153.3,153.2,135.0,133.5,110.1,101.7,97.1$, 91.1, 76.0, 73.2, 63.3, 60.8, 56.1, 53.8, 26.2, 24.6. HRMS calcd for $\mathrm{C}_{19} \mathrm{H}_{23} \mathrm{NO}_{9}\left(\mathrm{M}^{+}\right)$409.1373, found 409.1369 .

\section{Ketone 4z}

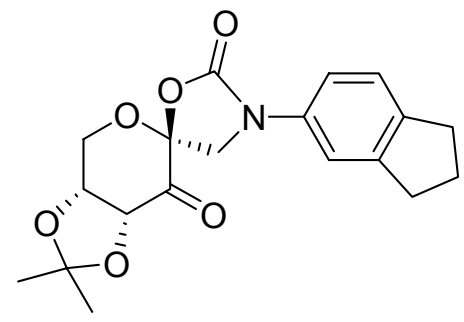

mp 78-80 ${ }^{\circ} \mathrm{C} ;[\alpha]^{20}{ }_{\mathrm{D}}=-45.7\left(c, 0.07, \mathrm{CHCl}_{3}\right) ; \mathrm{IR}(\mathrm{film}) 3450,1774 \mathrm{~cm}^{-1} ;{ }^{1} \mathrm{H} \mathrm{NMR}(300 \mathrm{MHz}$, $\left.\mathrm{CDCl}_{3}\right) \delta 7.43-7.38(\mathrm{~m}, 1 \mathrm{H}), 7.23-7.16(\mathrm{~m}, 2 \mathrm{H}), 4.87(\mathrm{~d}, J=5.4 \mathrm{~Hz}, 1 \mathrm{H}), 4.74(\mathrm{~d}, J=10.5 \mathrm{~Hz}$ 1H), 4.67-4.60 (m, 2H), $4.26(\mathrm{~d}, J=13.8 \mathrm{~Hz}, 1 \mathrm{H}), 3.74(\mathrm{~d}, J=10.5 \mathrm{~Hz}, 1 \mathrm{H}), 2.94-2.82(\mathrm{~m}, 4 \mathrm{H})$, 2.13-2.02 (m, 2H), $1.48(\mathrm{~s}, 3 \mathrm{H}), 1.43(\mathrm{~s}, 3 \mathrm{H}) ;{ }^{13} \mathrm{C} \mathrm{NMR}\left(100 \mathrm{MHz}, \mathrm{CDCl}_{3}\right) \delta$ 153.4, 145.6, $141.1,135.7,124.8,117.4,115.9,110.4,101.7,91.1,76.2,73.4,63.0,53.8,33.2,32.5,26.5$, 25.8, 24.9. Anal. Calcd for $\mathrm{C}_{19} \mathrm{H}_{21} \mathrm{NO}_{6}$ : C, 63.50; H, 5.89; N, 3.90. Found: C, 63.39; H, 5.76; N, 3.79 .

\section{Ketone 4aa}




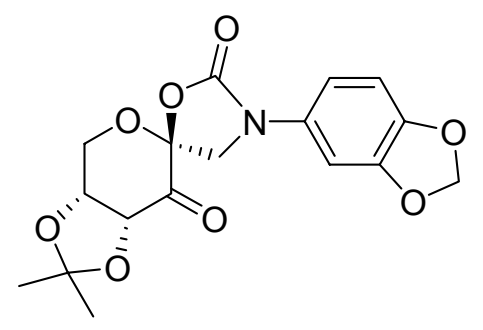

mp $138-140{ }^{\circ} \mathrm{C} ;[\alpha]^{20}{ }_{\mathrm{D}}=-34.8(c, 0.46, \mathrm{EtOH}) ; \mathrm{IR}\left(\right.$ film) $3374,1748 \mathrm{~cm}^{-1}$; hydrate: ${ }^{1} \mathrm{H}$ NMR $\left(300 \mathrm{MHz}, \mathrm{CDCl}_{3}\right) \delta 7.22(\mathrm{~s}, 1 \mathrm{H}), 6.76(\mathrm{~m}, 2 \mathrm{H}), 5.96(\mathrm{~s}, 2 \mathrm{H}), 4.48(\mathrm{~d}, J=9.9 \mathrm{~Hz}, 1 \mathrm{H}), 4.43(\mathrm{~m}$, 2H), 4.35-4.28 (m, 1H), $4.01(\mathrm{~d}, J=13.2 \mathrm{~Hz}, 1 \mathrm{H}), 3.81(\mathrm{brs}, 1 \mathrm{H}), 3.78(\mathrm{~d}, J=9.9 \mathrm{~Hz}, 1 \mathrm{H}), 3.56$ (brs, $1 \mathrm{H}), 1.59(\mathrm{~s}, 3 \mathrm{H}), 1.41(\mathrm{~s}, 3 \mathrm{H}) ;{ }^{13} \mathrm{C} \mathrm{NMR}\left(100 \mathrm{MHz}, \mathrm{CO}\left(\mathrm{CD}_{3}\right)_{2}\right) \delta$ 153.4, 149.0, 145.0, 134.0, 112.1, 110.5, 108.8, 102.4, 101.7, 92.4, 77.4, 74.5, 64.8, 55.1, 26.7, 25.0. HRMS calcd for $\mathrm{C}_{17} \mathrm{H}_{17} \mathrm{NO}_{8}\left(\mathrm{M}^{+}\right)$363.0954, found 363.0951.

\section{Ketone $4 b b$}

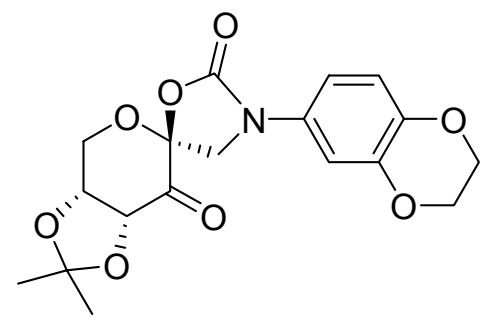

mp 140-142 ${ }^{\circ} \mathrm{C} ;[\alpha]^{20}{ }_{\mathrm{D}}=-33.3(c, 0.38, \mathrm{EtOH}) ; \mathrm{IR}\left(\right.$ film) $3400,1749 \mathrm{~cm}^{-1}$; hydrate: ${ }^{1} \mathrm{H}$ NMR $\left(300 \mathrm{MHz}, \mathrm{CDCl}_{3}\right) \delta 7.06(\mathrm{~d}, J=2.4 \mathrm{~Hz}, 1 \mathrm{H}), 6.97(\mathrm{dd}, \mathrm{J}=8.7,2.4 \mathrm{~Hz}, 1 \mathrm{H}), 6.83(\mathrm{~d}, \mathrm{~J}=8.7 \mathrm{~Hz}$, 1H), 4.47 (d, $J=10.2 \mathrm{~Hz}, 1 \mathrm{H}), 4.42(\mathrm{~s}, 2 \mathrm{H}), 4.32$ (dd, $J=13.2,1.8 \mathrm{~Hz}, 1 \mathrm{H}), 4.26-4.20$ (m, 4H), $4.01(\mathrm{~d}, J=13.2 \mathrm{~Hz}, 1 \mathrm{H}), 3.77(\mathrm{~d}, J=10.2 \mathrm{~Hz}, 1 \mathrm{H}), 3.72$ (brs, 1H), 3.46 (brs, 1H), 1.59 (s, 3H), $1.41(\mathrm{~s}, 3 \mathrm{H}) ;{ }^{13} \mathrm{C}$ NMR $\left(100 \mathrm{MHz}, \mathrm{CO}\left(\mathrm{CD}_{3}\right)_{2}\right) \delta 153.3,144.7,141.2,133.2,118.0,112.2,110.4$, 108.6, 102.4, 92.4, 77.4, 74.5, 65.4, 65.1, 64.7, 54.8, 26.7, 25.0. Anal. Calcd for $\mathrm{C}_{18} \mathrm{H}_{19} \mathrm{NO}_{8}$ : C, 57.29; H, 5.08; N, 3.71. Found: C, 57.15; H, 5.15; N, 3.53. 


\section{Ketone 4cc}

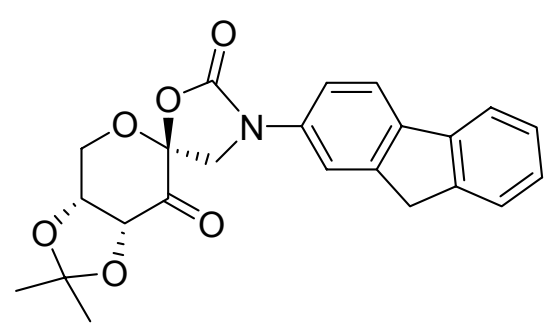

$\operatorname{mp~} 195-197{ }^{\circ} \mathrm{C} ;[\alpha]^{20}{ }_{\mathrm{D}}=-82.6\left(c, 0.12, \mathrm{CHCl}_{3}\right) ; \mathrm{IR}\left(\right.$ film) $3400,1757 \mathrm{~cm}^{-1}$; hydrate: ${ }^{1} \mathrm{H}$ NMR $\left(300 \mathrm{MHz}, \mathrm{CDCl}_{3}\right) \delta$ 7.75-7.64 (m, 3H), 7.48-7.24 (m, 4H), $4.63(\mathrm{~d}, J=10.2 \mathrm{~Hz}, 1 \mathrm{H}), 4.46(\mathrm{~m}$, 2H), 4.35 (dd, $J=12.9,2.1 \mathrm{~Hz}, 1 \mathrm{H}), 4.05$ (brs, 1H), 4.02 (d, $J=12.9 \mathrm{~Hz}, 1 \mathrm{H}), 3.88$ (d, $J=10.2$ $\mathrm{Hz}, 1 \mathrm{H}), 3.88$ (brs, $1 \mathrm{H}), 3.81(\mathrm{~s}, 2 \mathrm{H}), 1.61(\mathrm{~s}, 3 \mathrm{H}), 1.42(\mathrm{~s}, 3 \mathrm{H}) ;{ }^{13} \mathrm{C} \mathrm{NMR}\left(100 \mathrm{MHz}, \mathrm{CDCl}_{3}\right) \delta$ $153.3,144.5,143.3,141.1,138.6,136.2,127.0,126.7,125.2,120.3,119.9,117.8,116.2,110.5$ 101.9, 91.3, 76.2, 73.4, 63.2, 53.7, 37.2, 26.5, 24.9. HRMS calcd for $\mathrm{C}_{23} \mathrm{H}_{22} \mathrm{NO}_{6}\left(\mathrm{M}^{+}+1\right)$ 408.1447, found 408.1439. 
The chromatograms for the determination of enantiomeric excess of the epoxides.

(R)-Styrene oxide (Table 2, Entry 1)<smiles>c1ccc(C2CO2)cc1</smiles>

GC Cond.: Column: Chiraldex BDM (Cat. No. 77023), Adv. Separation Technologies, Inc. Oven: $80^{\circ} \mathrm{C}$; Carrier: Helium, head pressure 25 psi: Detection:FID $250^{\circ} \mathrm{C}$
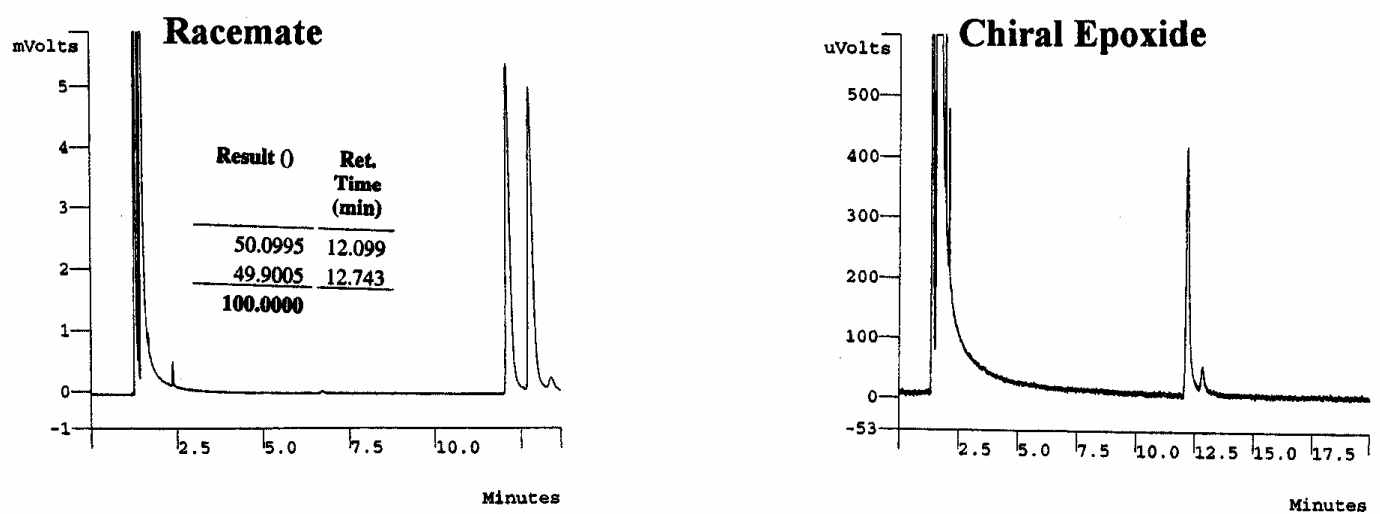

$\underset{F}{2-F l u o r o s t y r e n e ~ o x i d e ~(T a b l e ~ 2, ~ E n t r y ~ 2) ~} \underset{\text { No }}{\text { Peak }} \quad$ Peak Name $\quad$ Result $0 \quad \begin{gathered}\text { Ret. } \\ \text { Time }\end{gathered}$<smiles>Fc1ccccc1C1CO1</smiles>

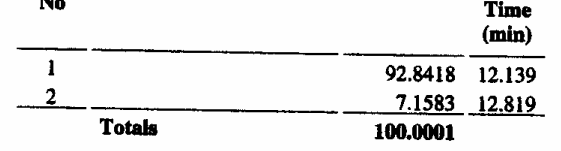

GC Cond.: Column: Chiraldex BDM (Cat. No. 77023), Adv. Separation Technologies, Inc.
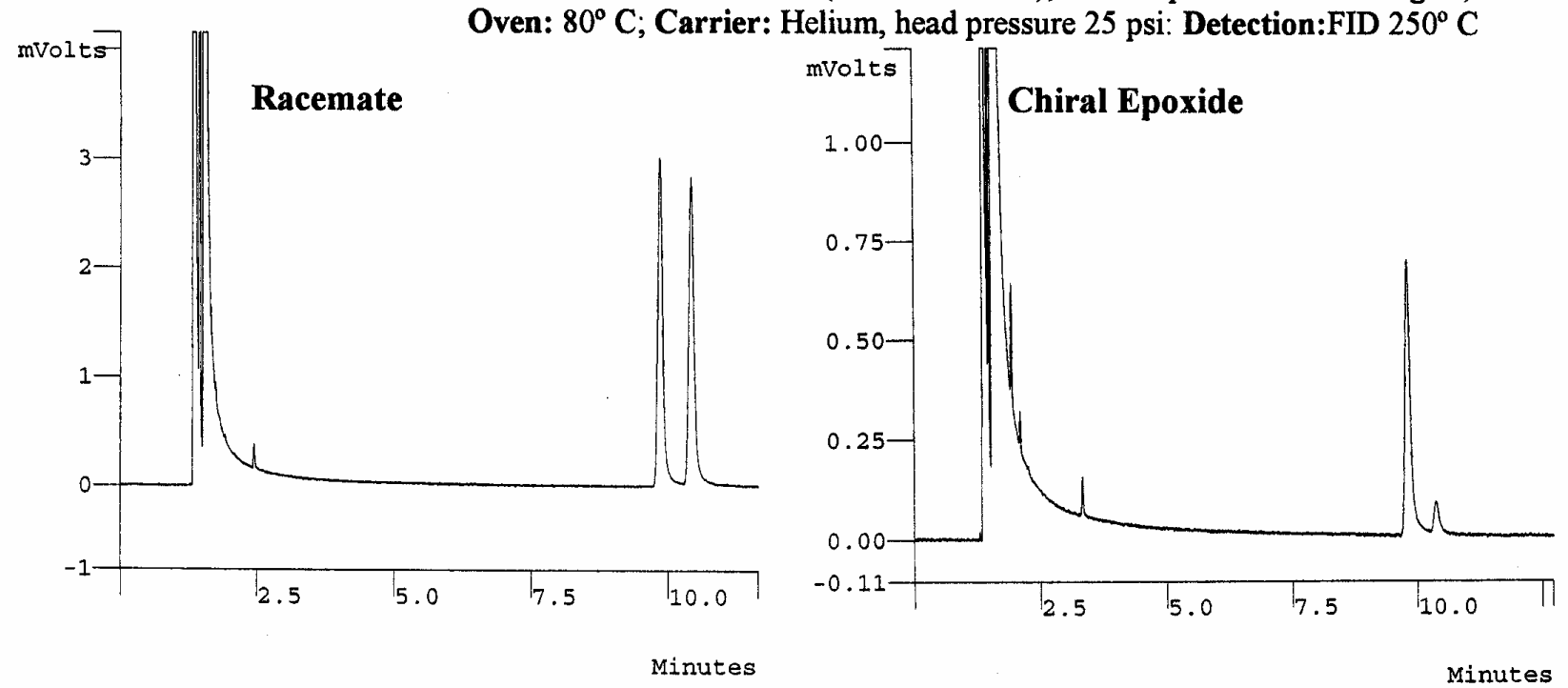

Minutes

\begin{tabular}{|c|c|c|c|}
\hline $\begin{array}{l}\text { ?eak } \\
\text { No }\end{array}$ & Peak Name & Result () & $\begin{array}{l}\text { Ret. } \\
\text { Time } \\
\text { (min) }\end{array}$ \\
\hline 1 & & 50.3680 & 9.824 \\
\hline 2 & & 49.6320 & 10.394 \\
\hline
\end{tabular}

\begin{tabular}{ll}
$\begin{array}{l}\text { Peak } \\
\text { No }\end{array}$ & Peak Name \\
\hline 1 \\
\hline 2
\end{tabular}


(R)-4-Chlorostyrene oxide (Table 2, Entry 3)

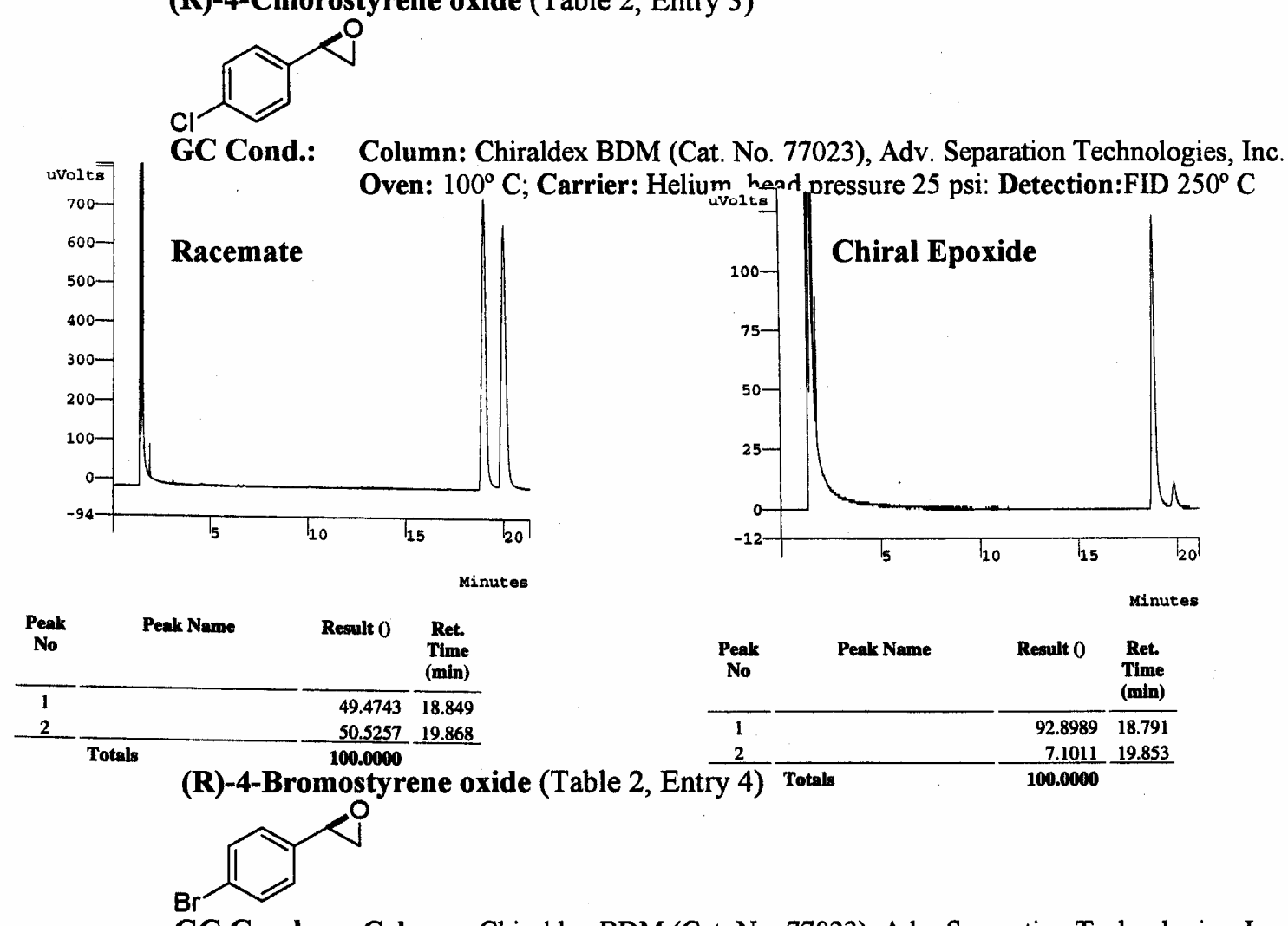

GC Cond.: Column: Chiraldex BDM (Cat. No. 77023), Adv. Separation Technologies, Inc.

Oven: $110^{\circ} \mathrm{C}$; Carrier: Helium, head pressure 25 psi: Detection:FID $250^{\circ} \mathrm{C}$
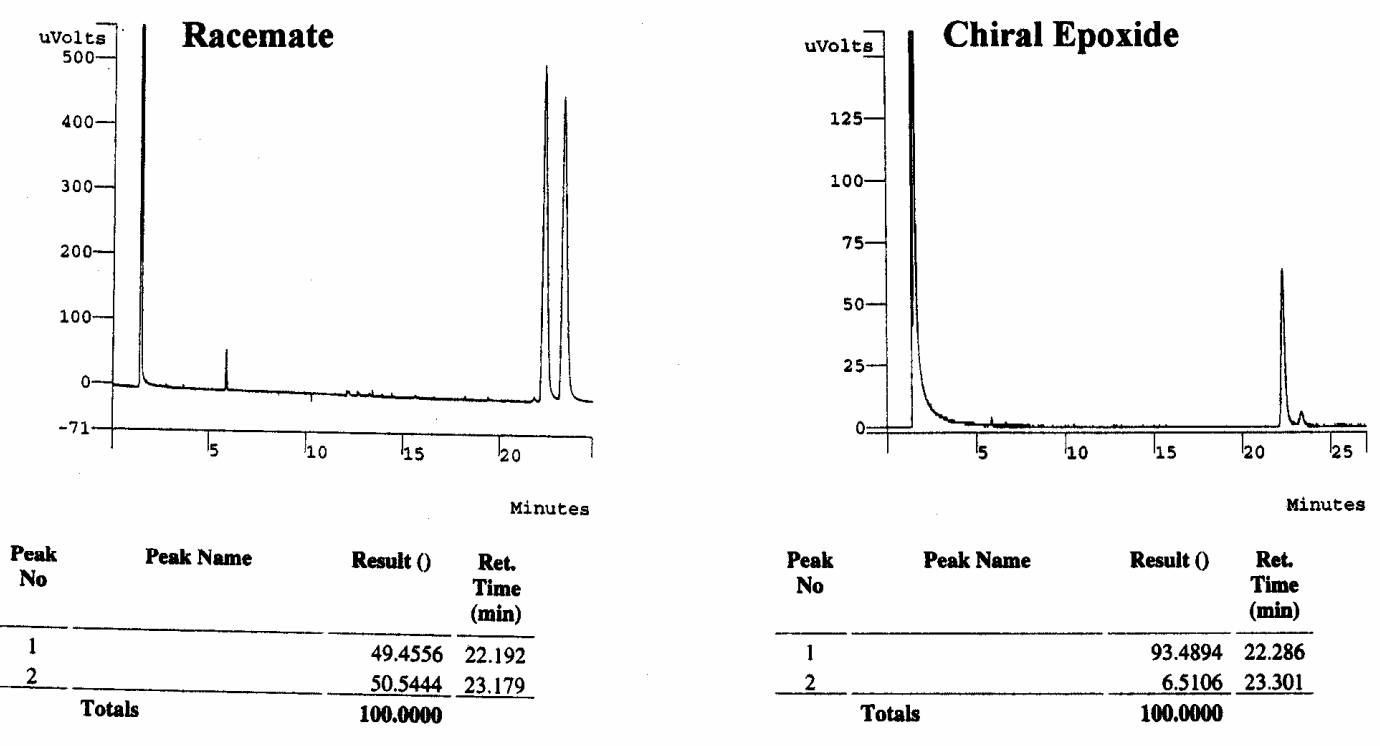
(R)-4-Cyanoostyrene oxide (Table 2, Entry 5)

GC Cond.: Column: Chiraldex BDM (Cat. No. 77023), Adv. Separation Technologies, Inc
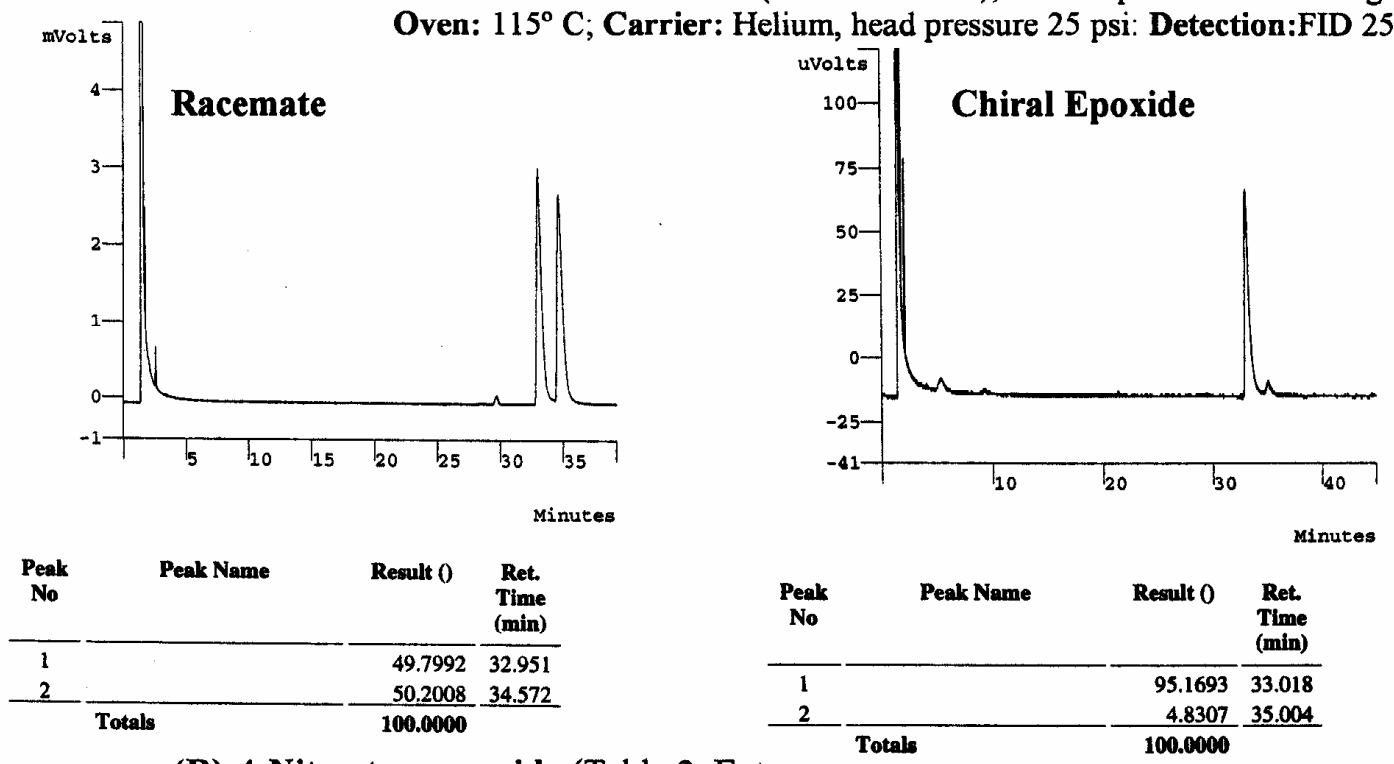

(R)-4-Nitrostyrene oxide (Table 2, Entry o)<smiles>O=[N+]([O-])c1ccc(C2CO2)cc1</smiles>

GC Cond.: Column: Chiraldex BDM (Cat. No. 77023), Adv. Separation Technologies, Inc.
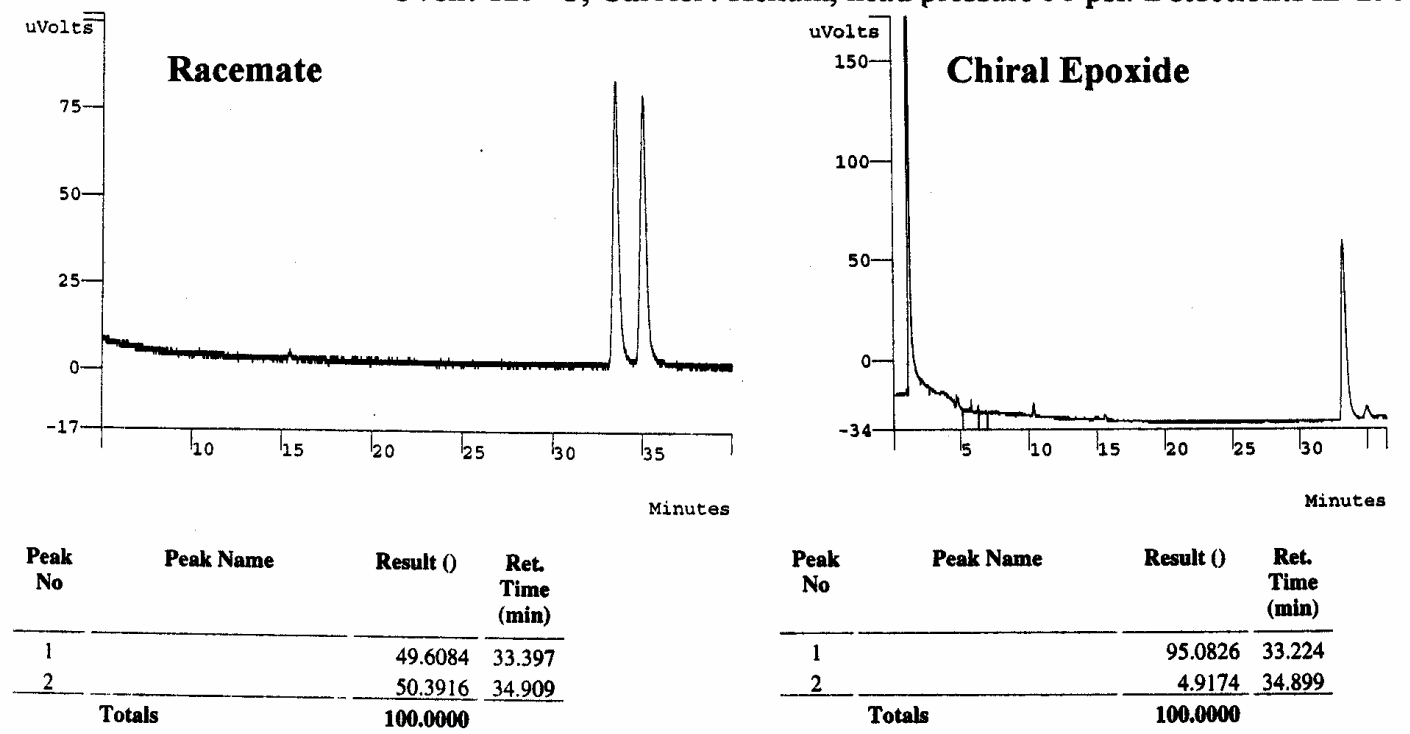


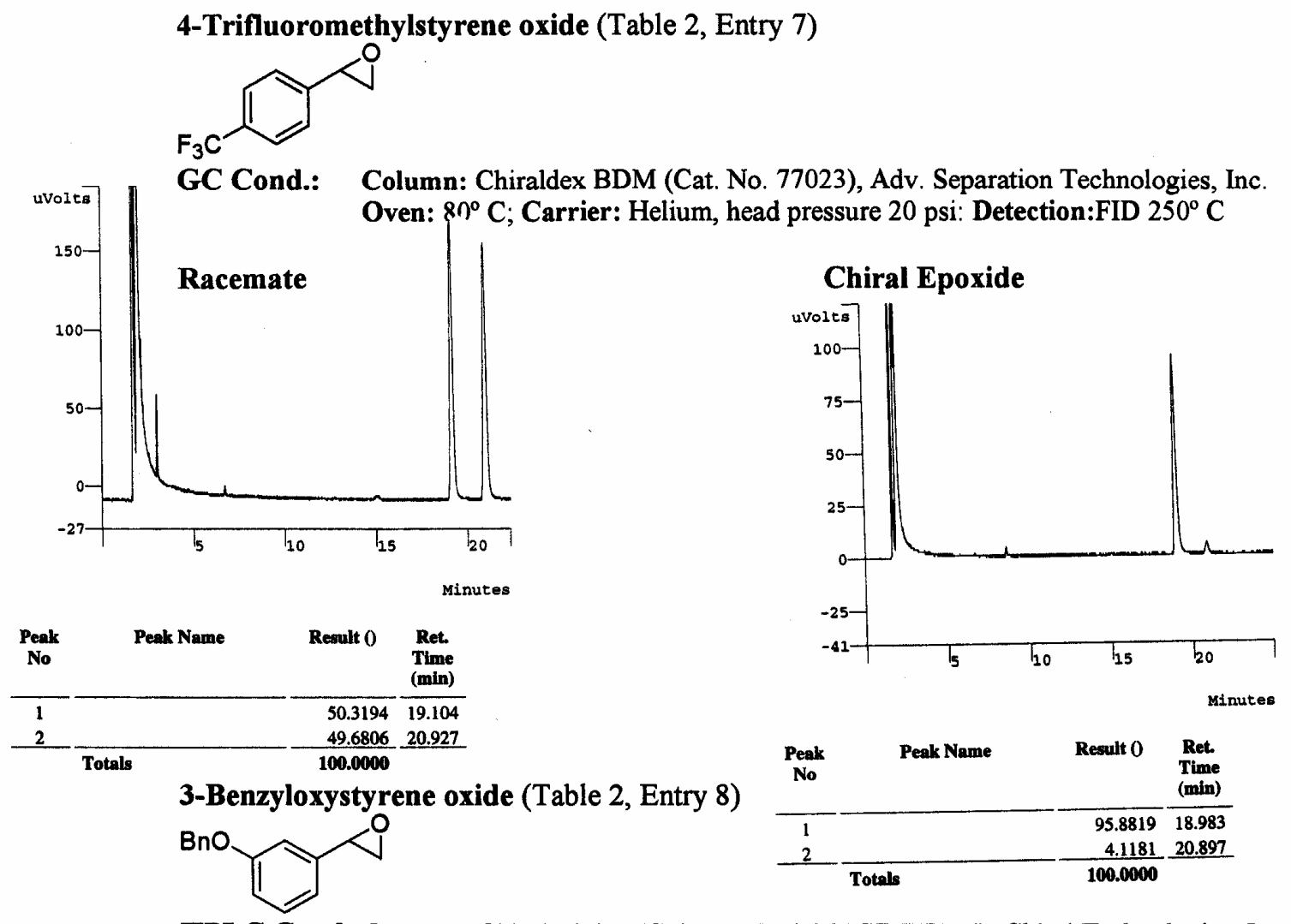

HPLC Cond.:Column: Chiralcel AD (Column No.AD00CE-BE096), Chiral Technologies, Inc. Eluent: Hexane/IPA (95/5); Flow Rate: $(1.0 \mathrm{~mL} / \mathrm{min})$ Detection:UV 270

Racemate

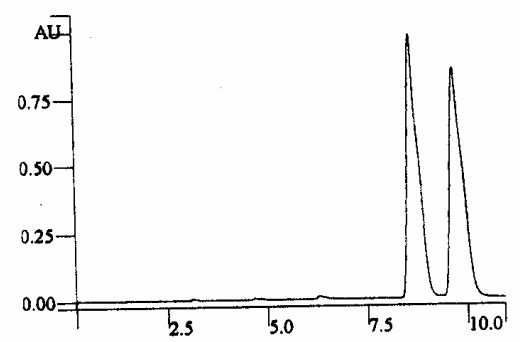

\section{Chiral Epoxide}
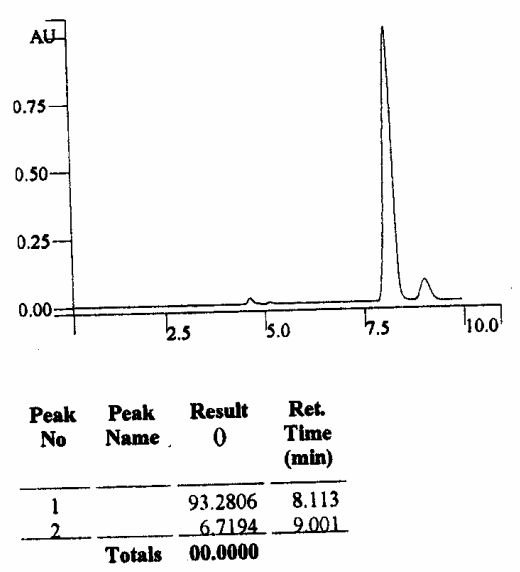
3-Methylstyrene oxide (Table 2, Entry 9)

Me

GC Cond.: Column: Chiraldex BDM (Cat. No. 77023), Adv. Separation Technologies, Inc. Oven: $60^{\circ} \mathrm{C}$; Carrier: Helium, head pressure 20 psi: Detection:FID $250^{\circ} \mathrm{C}$
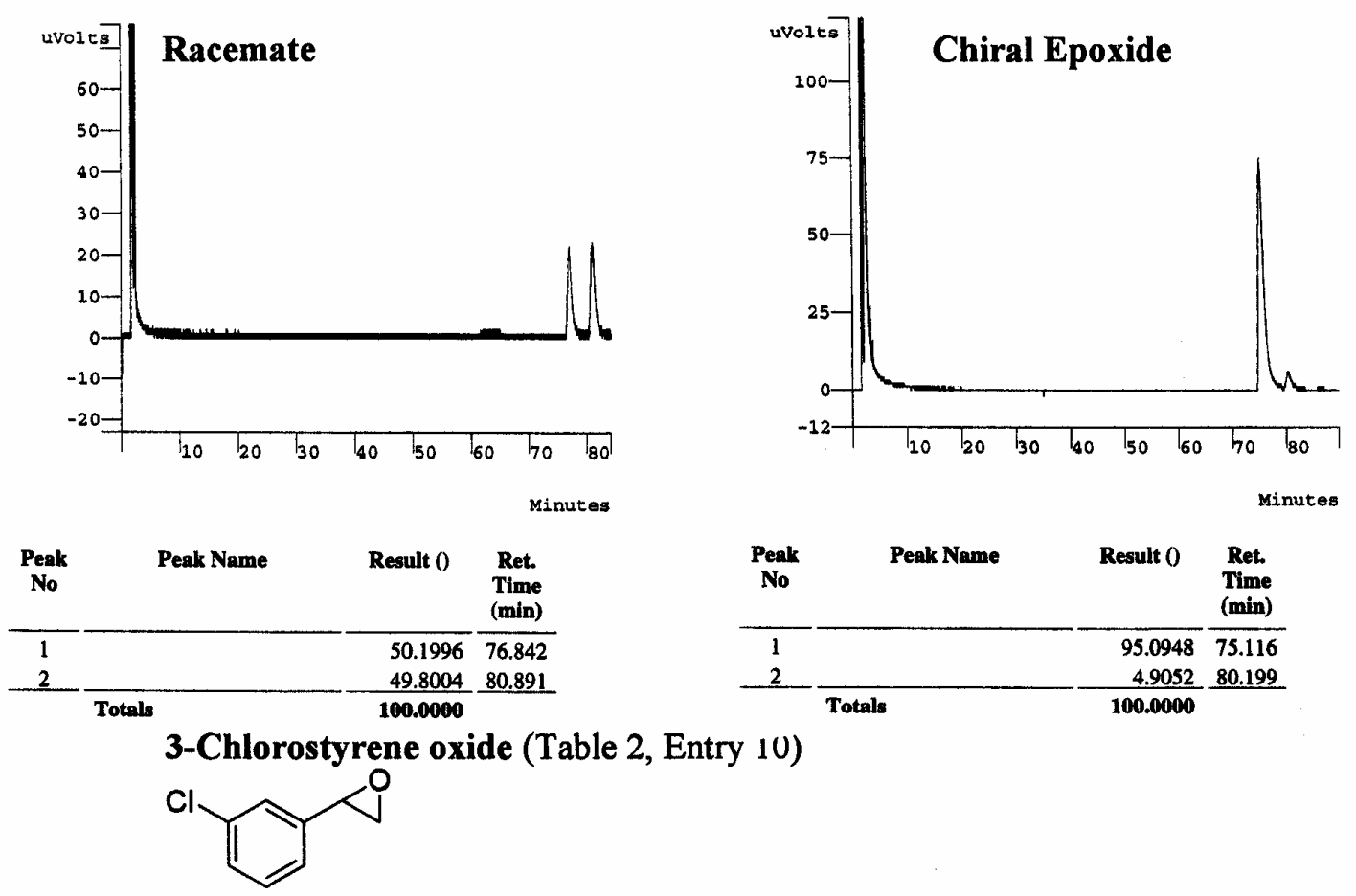

HPLC Cond.: Column: Chiralcel OD(Column No.OD00CE-DL010), Chiral Technologies, Inc. Eluent: Hexane/IPA (99/1); Flow Rate: (1.0 mL/min) Detection:UV 220
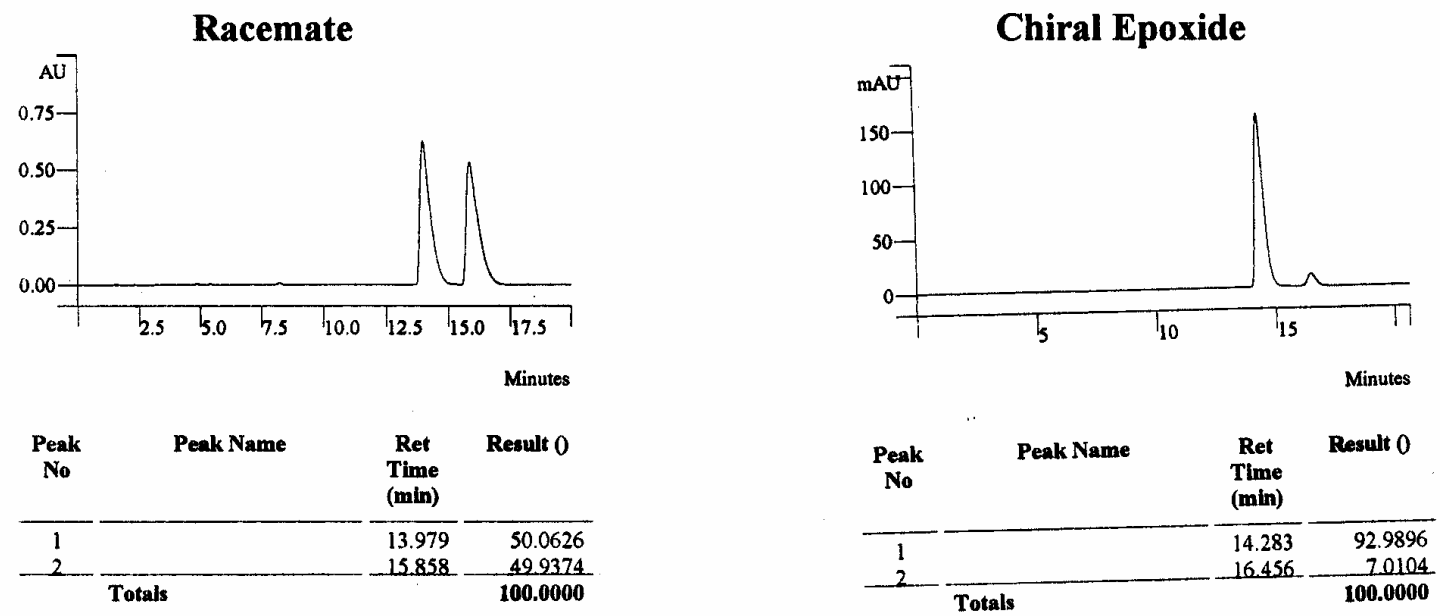
3-Nitrostyrene oxide (Table 2, Entry 11)<smiles>O=[N+]([O-])c1cccc(C2CO2)c1</smiles>

HPLC Cond.: Column: Chiralcel OD(Column No.OD00CE-DL010), Chiral Technologies, Inc. Eluent: Hexane/IPA (95/5); Flow Rate: (1.0 mL/min) Detection:UV 254

Racemate
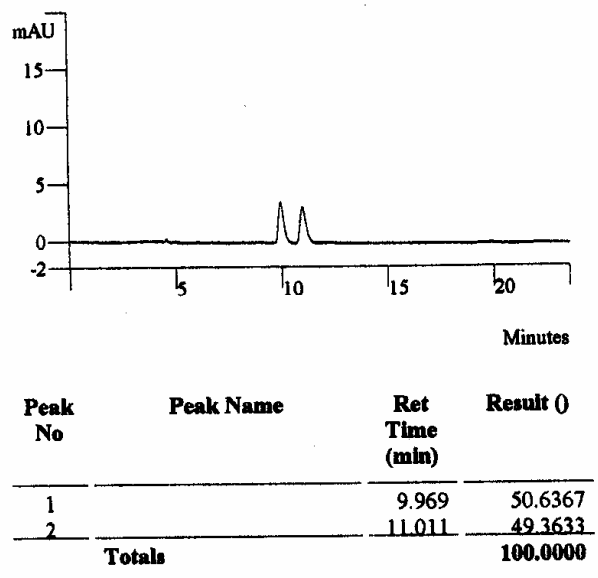

\section{Chiral Epoxide}
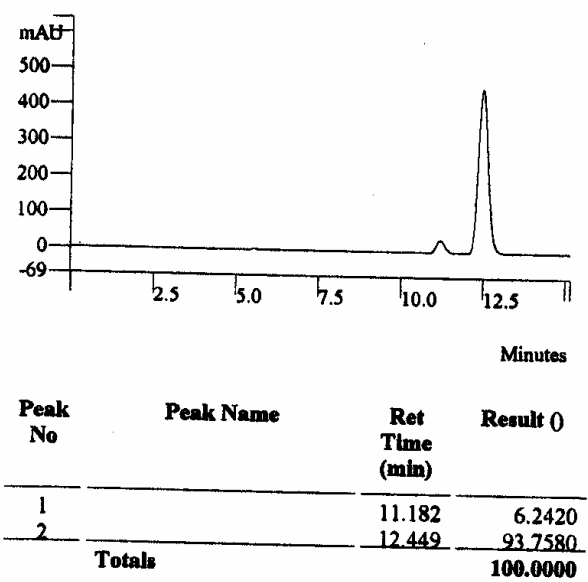

2-Naphthalene oxide (Table 2, Entry 12)<smiles>c1ccc2cc(C3CO3)ccc2c1</smiles>

HPLC Cond.: Column: Chiralcel OJ(Column No.OJ00CE-DE008), Chiral Technologies, Inc. Eluent: Hexane/IPA (100/0); Flow Rate: $(1.0 \mathrm{~mL} / \mathrm{min})$ Detection:UV 210
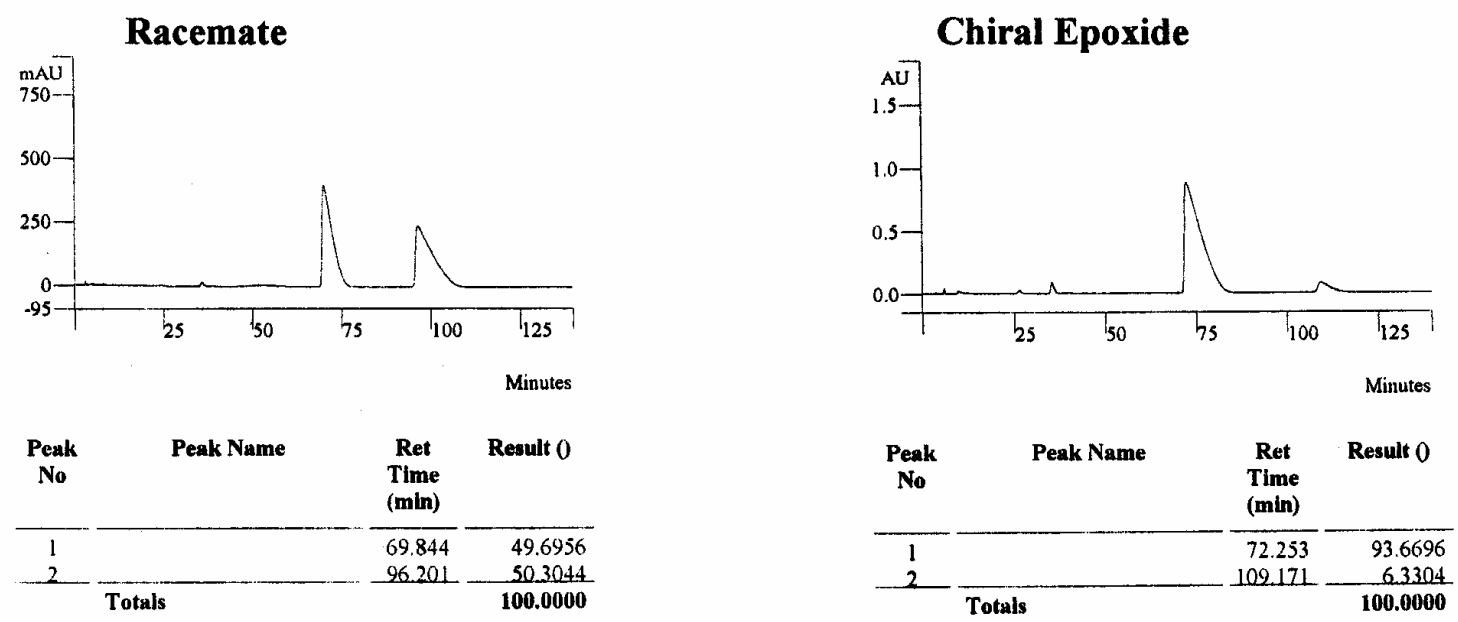


\section{3,4-Dichlororostyrene oxide (Table 2, Entry 13)}<smiles>Clc1ccc(C2CO2)cc1Cl</smiles>

GC Cond.: Column: Chiraldex BDM (Cat. No. 77023), Adv. Separation Technologies, Inc.
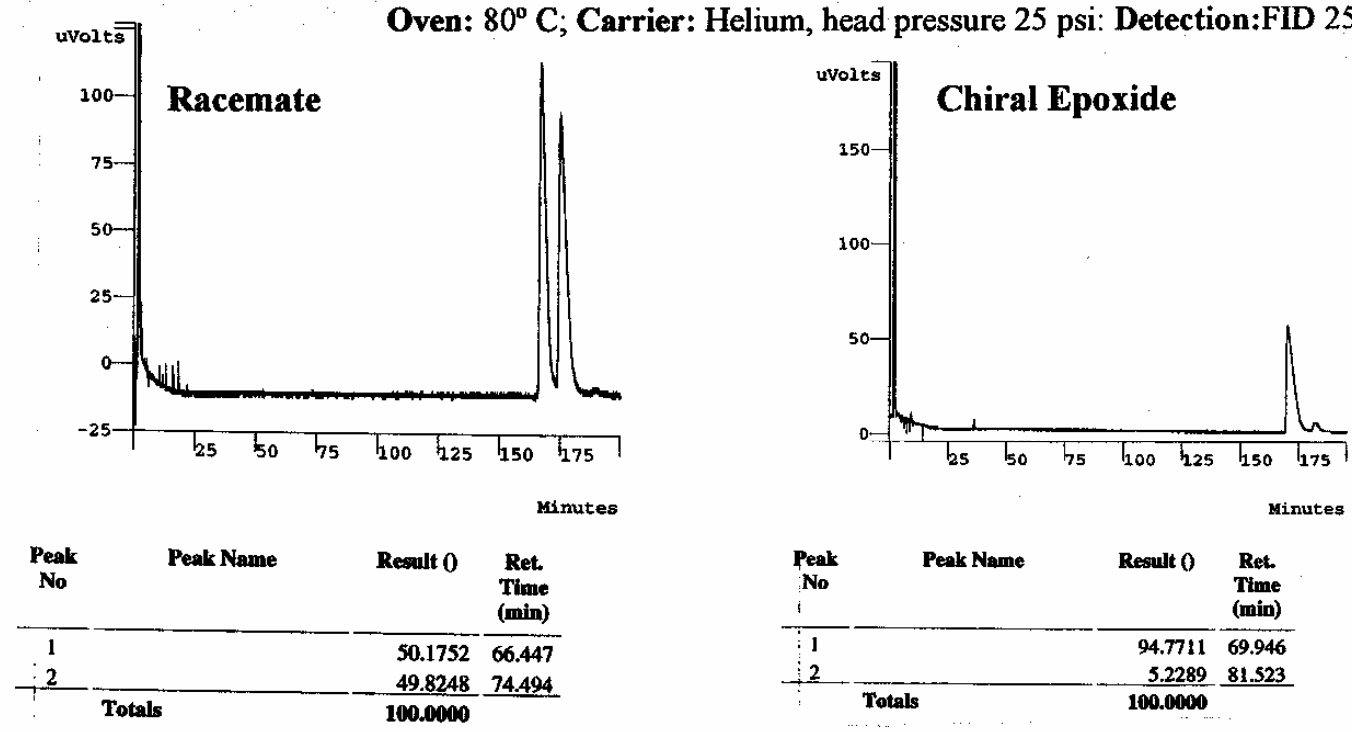

3,5-Dimethylstyrene oxide (Table 2, Entry 14)<smiles>Cc1cc(C)cc(C2CO2)c1</smiles>

GC Cond.: Column: Chiraldex BDM (Cat. No. 77023), Adv. Separation Technologies, Inc. Oven: $70^{\circ} \mathrm{C}$; Carrier: Helium, head pressure 20 psi: Detection:FID $250^{\circ} \mathrm{C}$

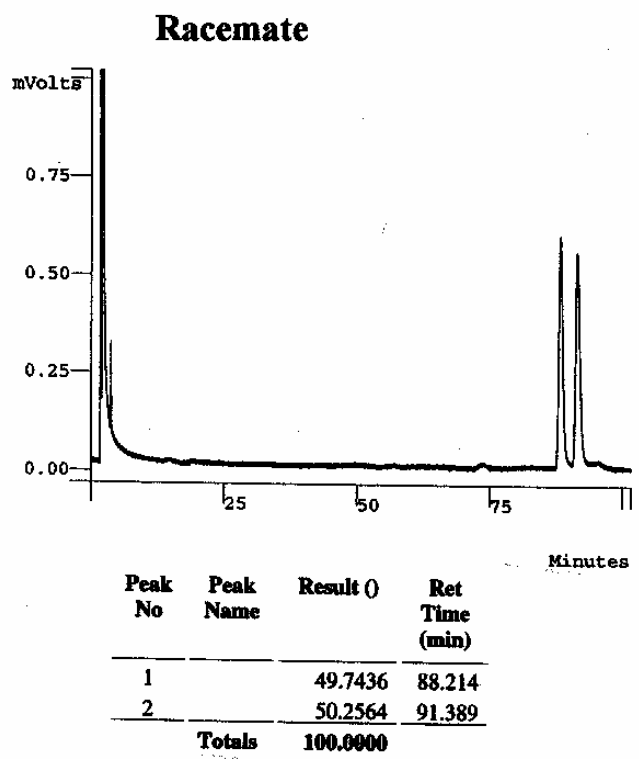

\section{Chiral Epoxide}
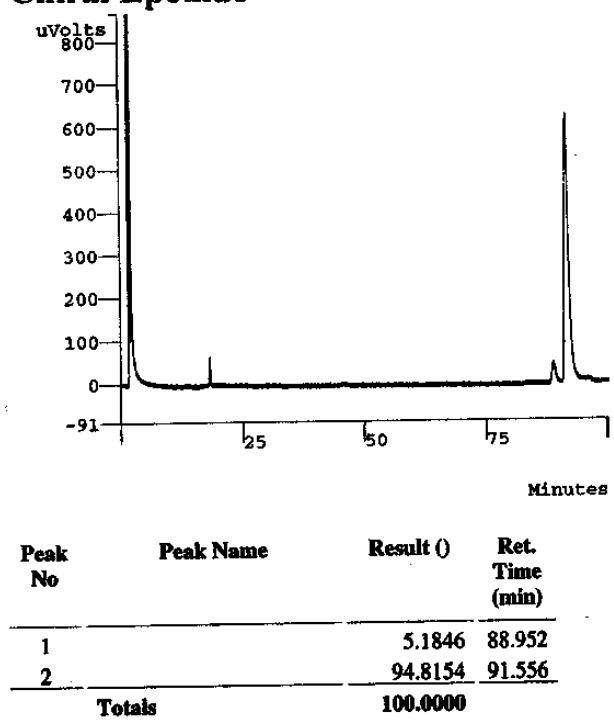

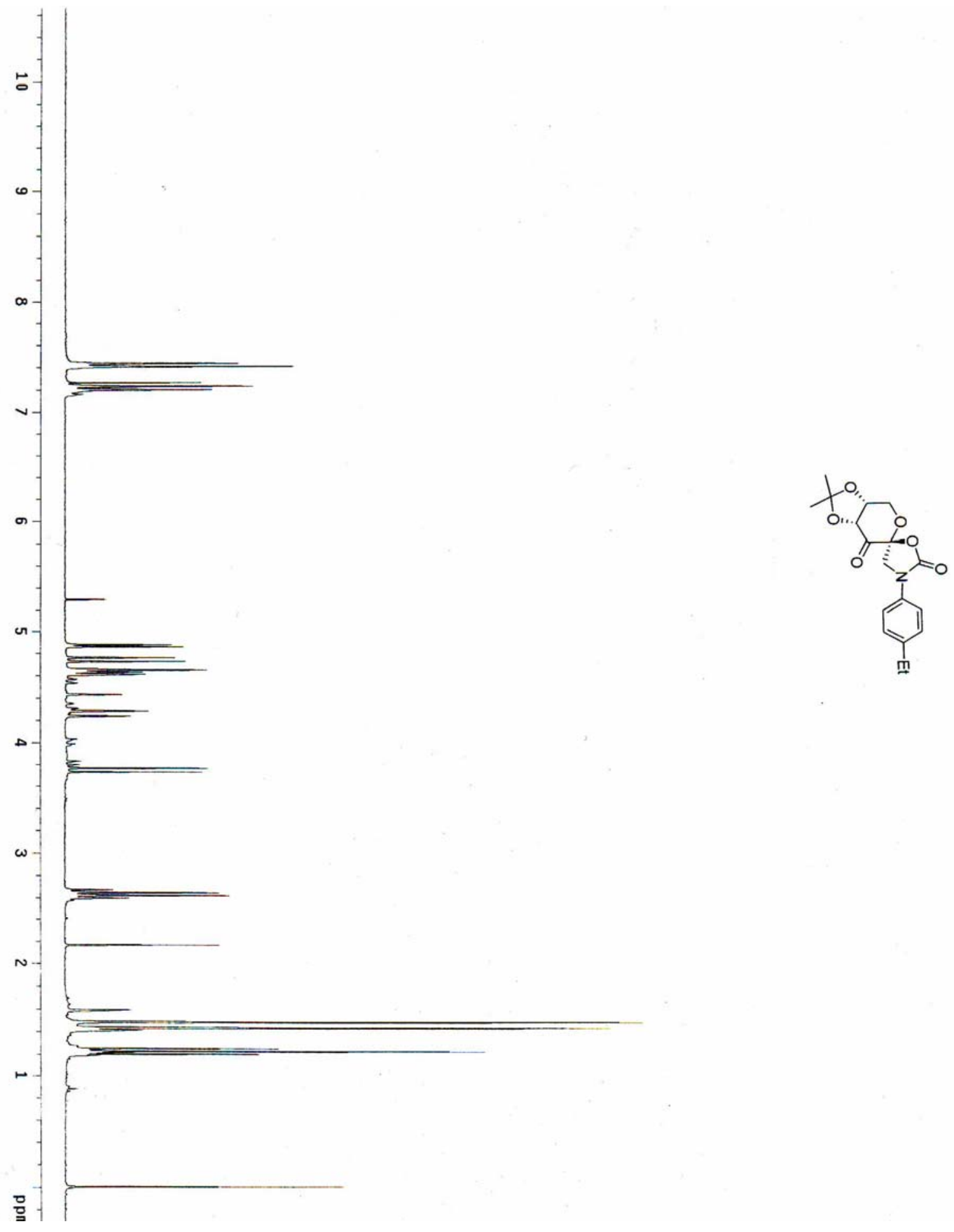

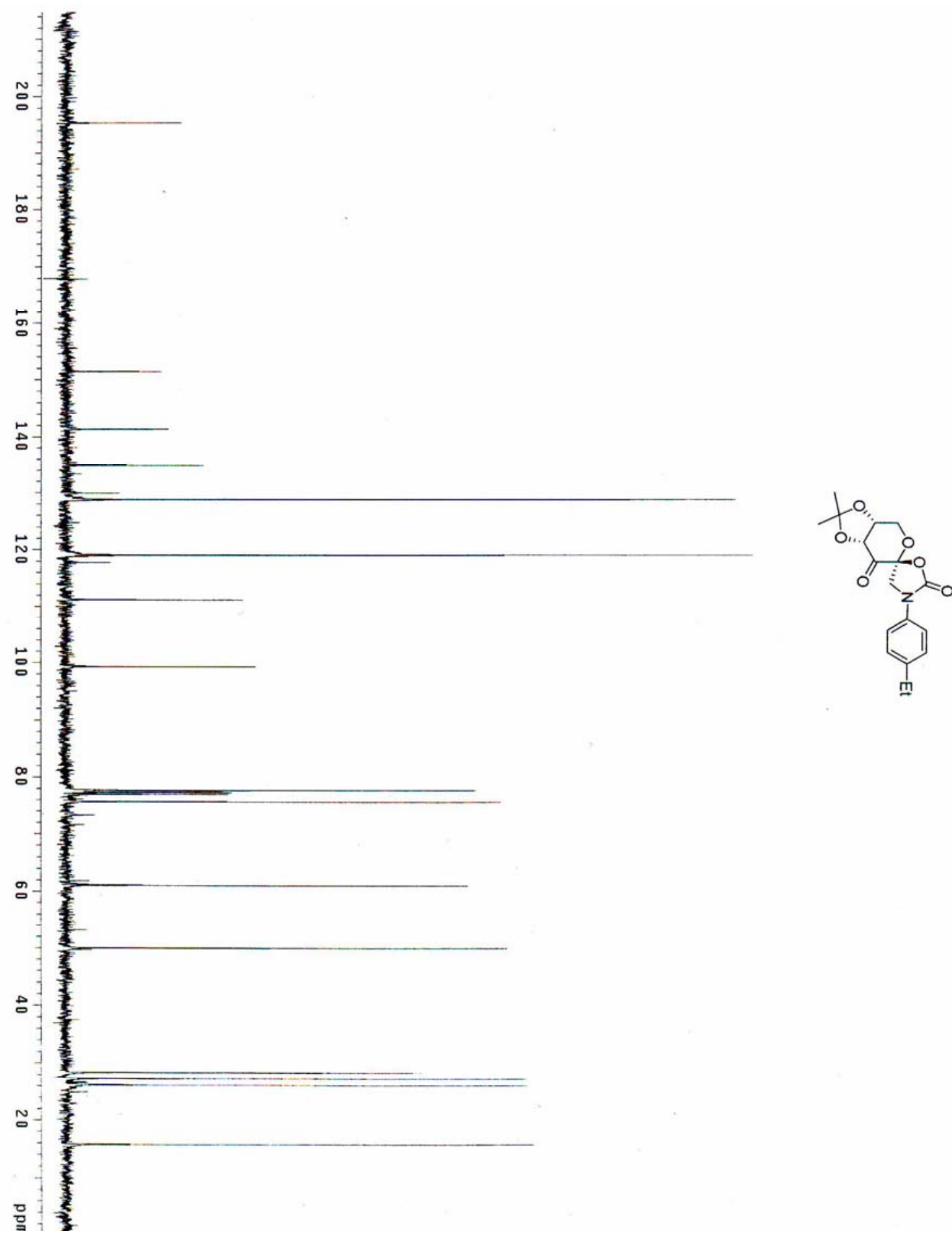


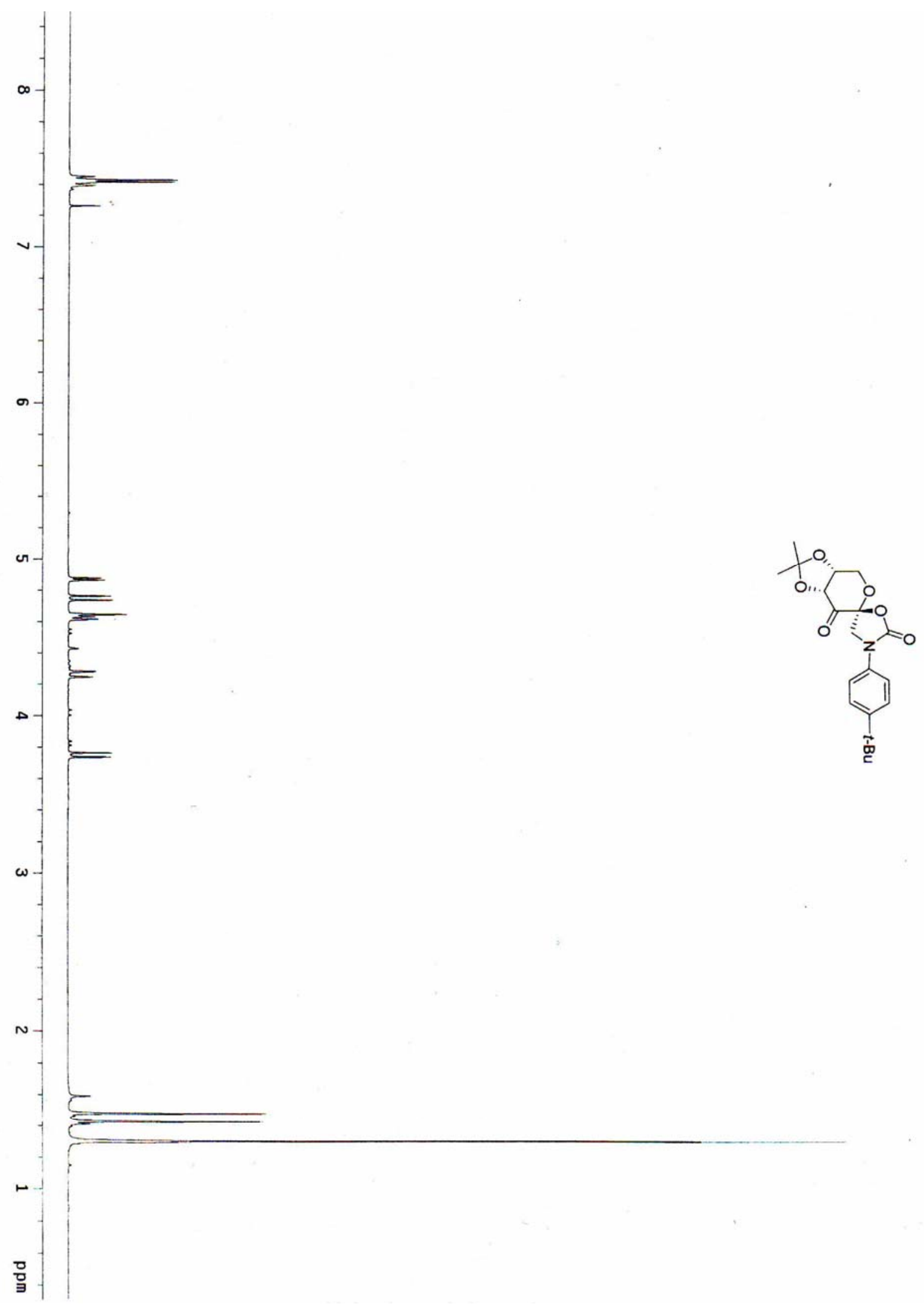




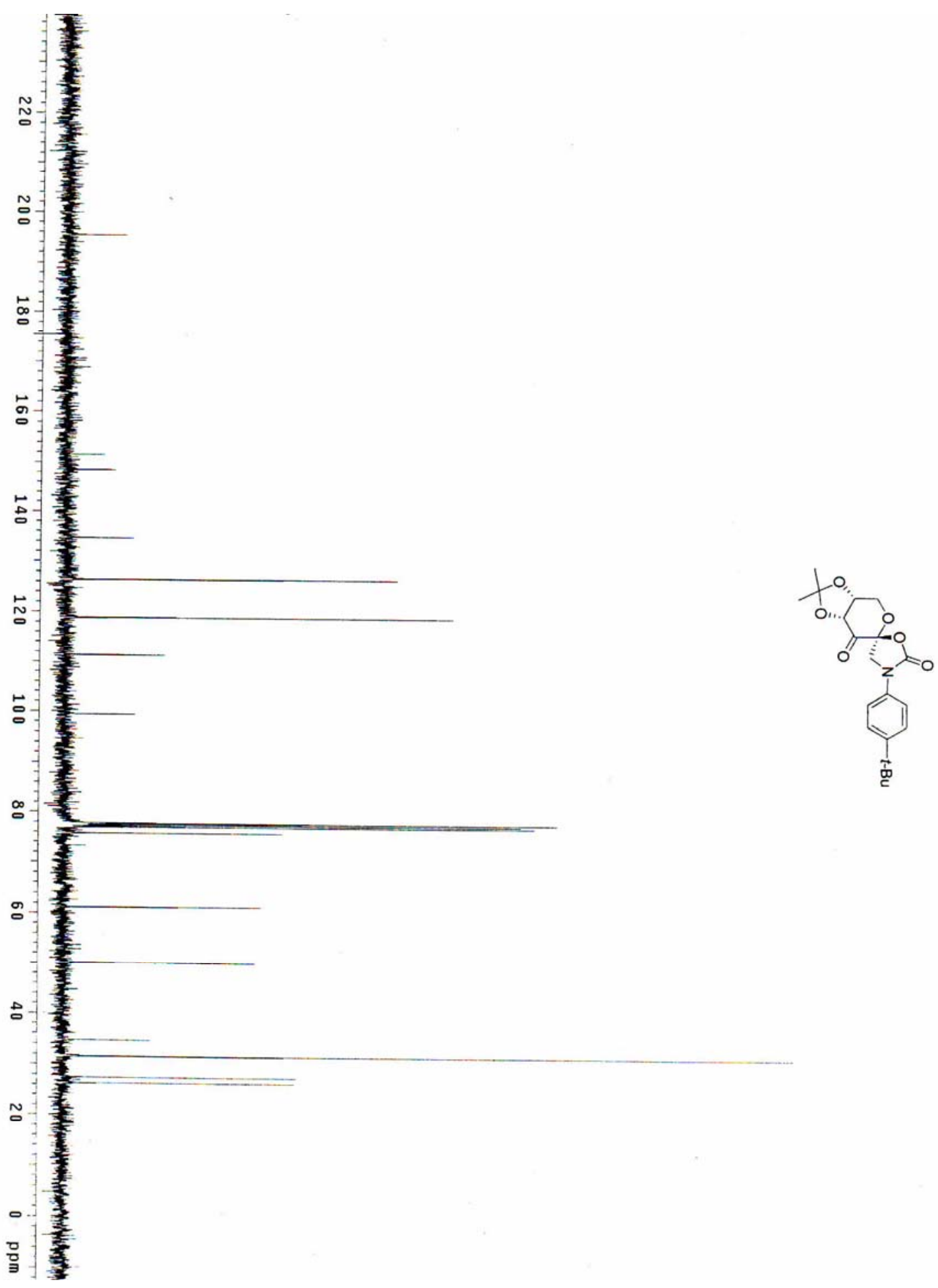



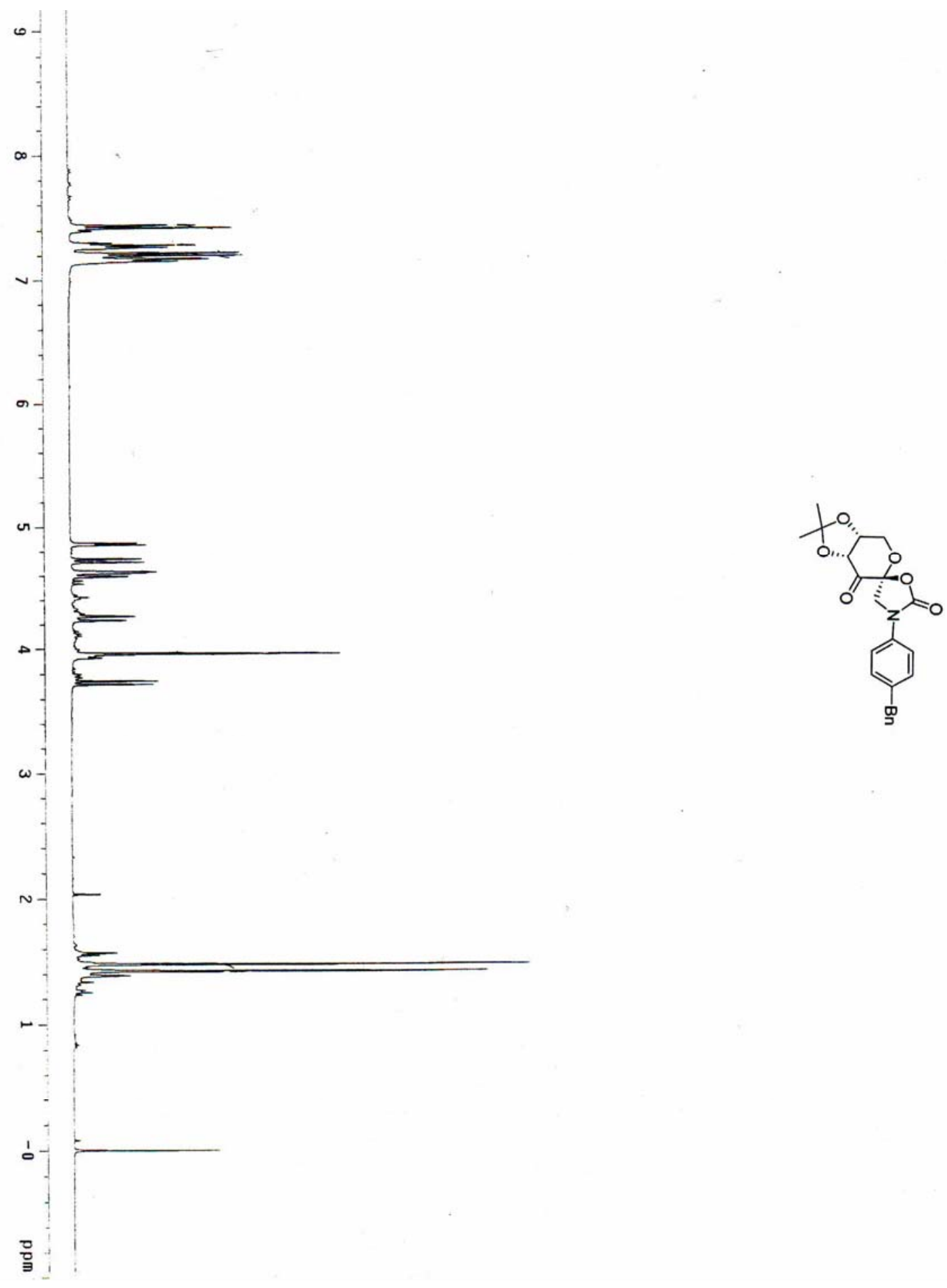


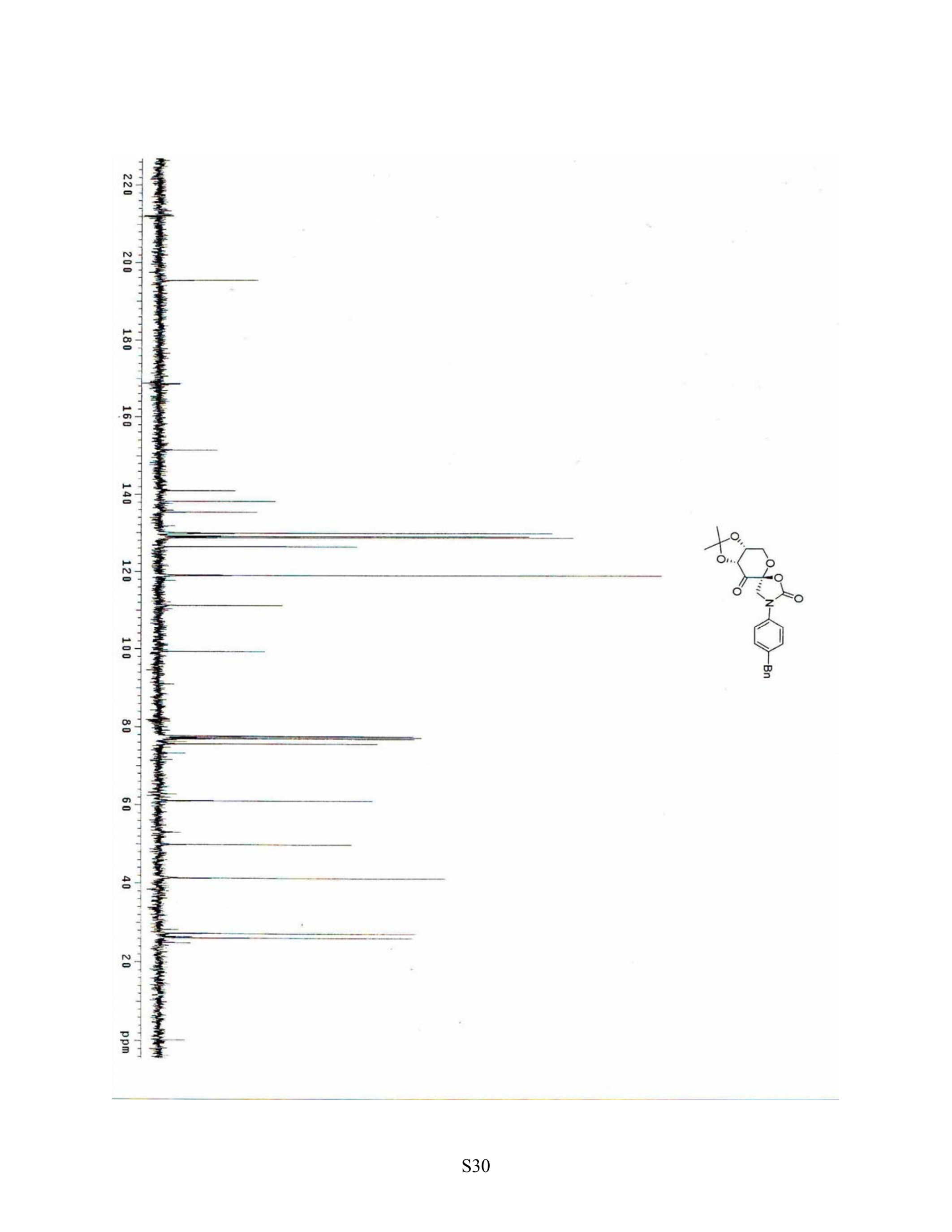




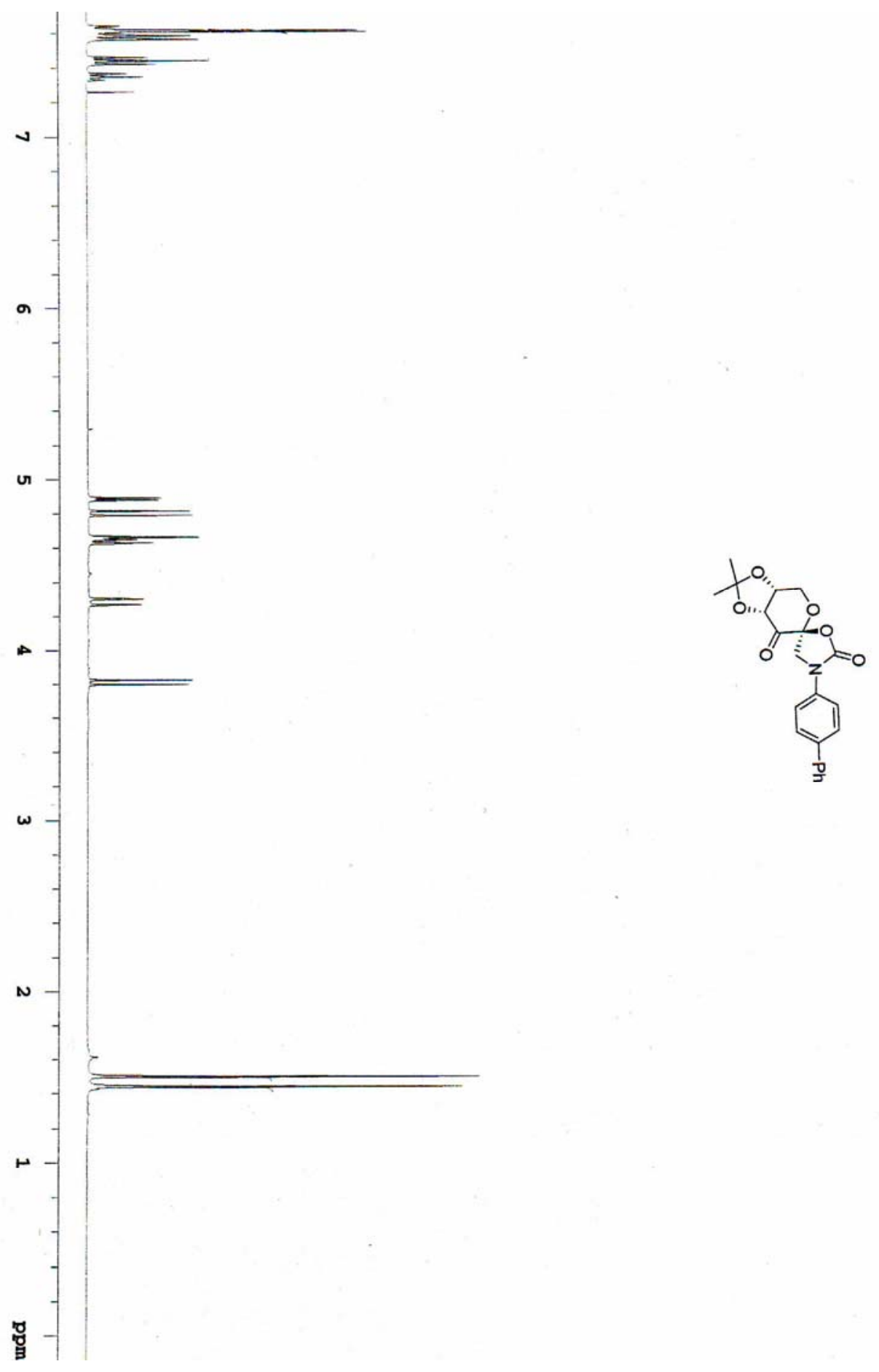



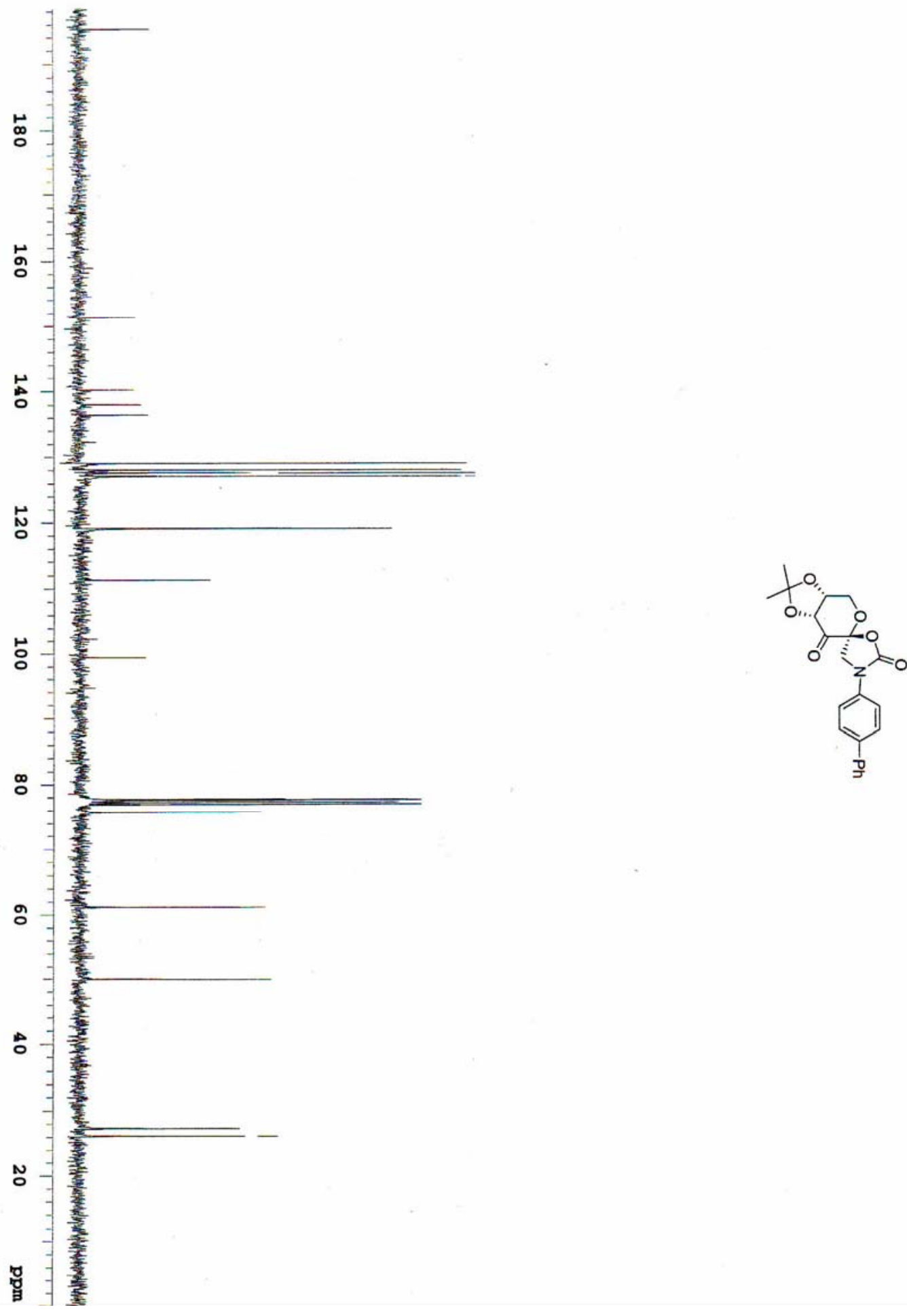

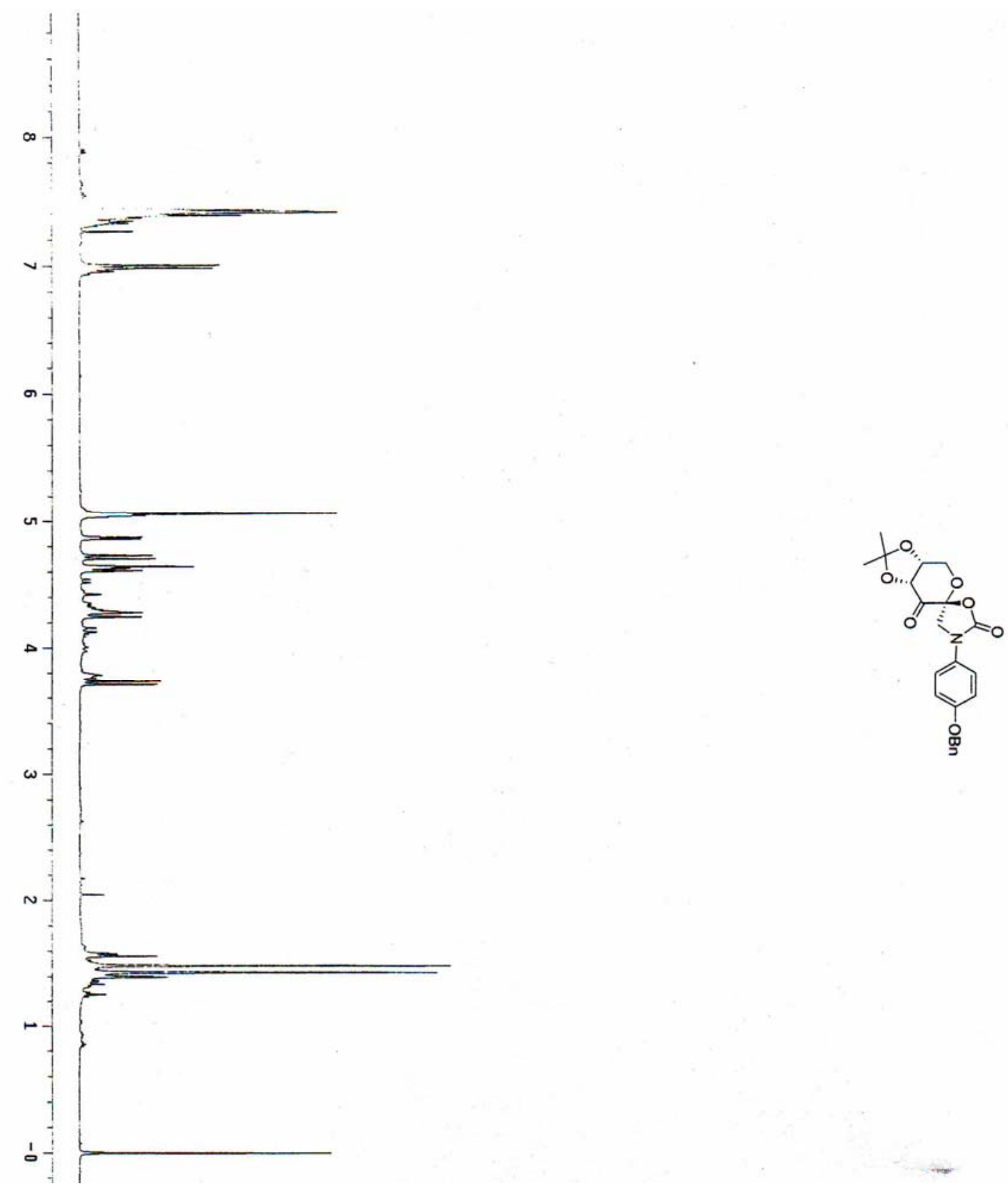


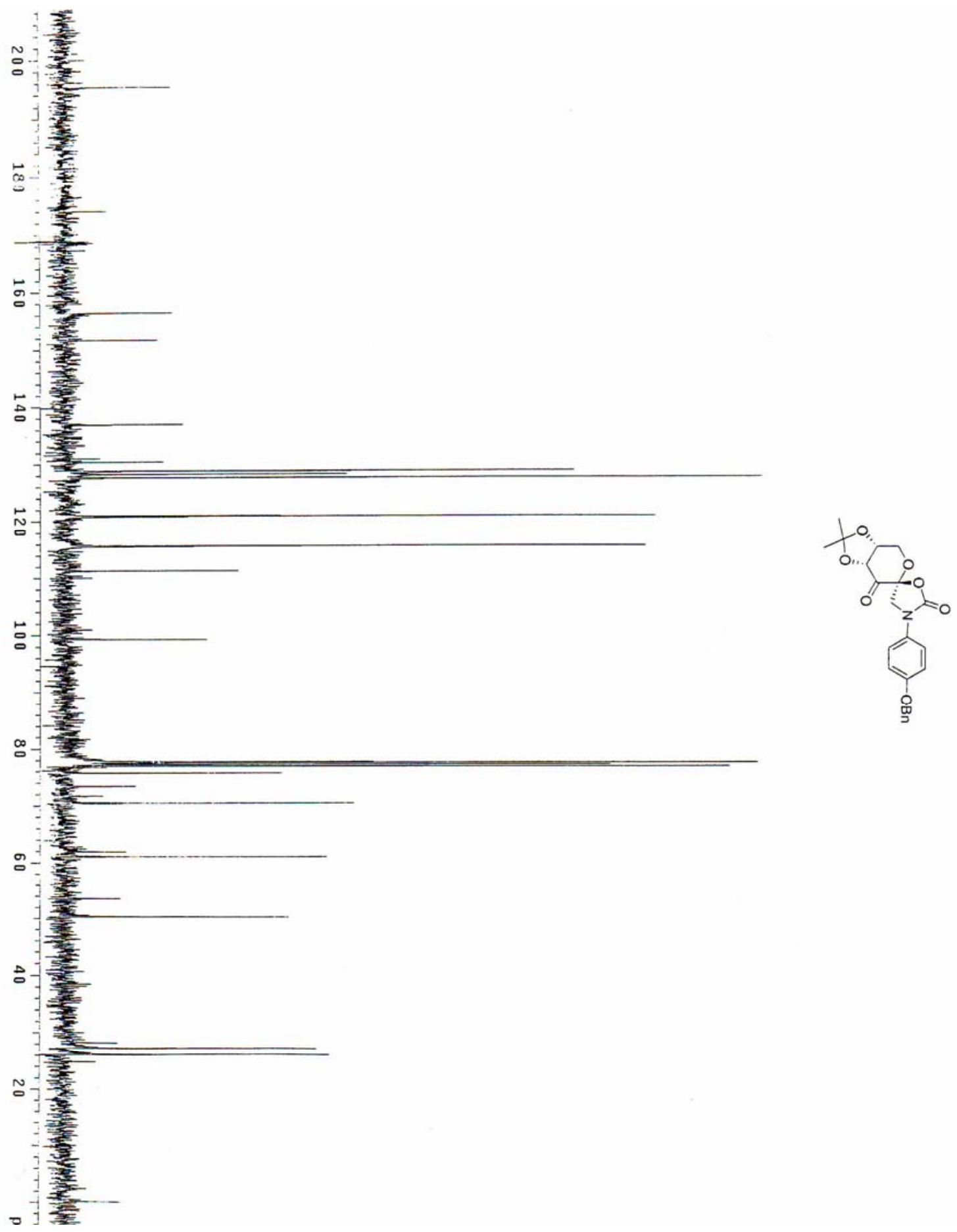




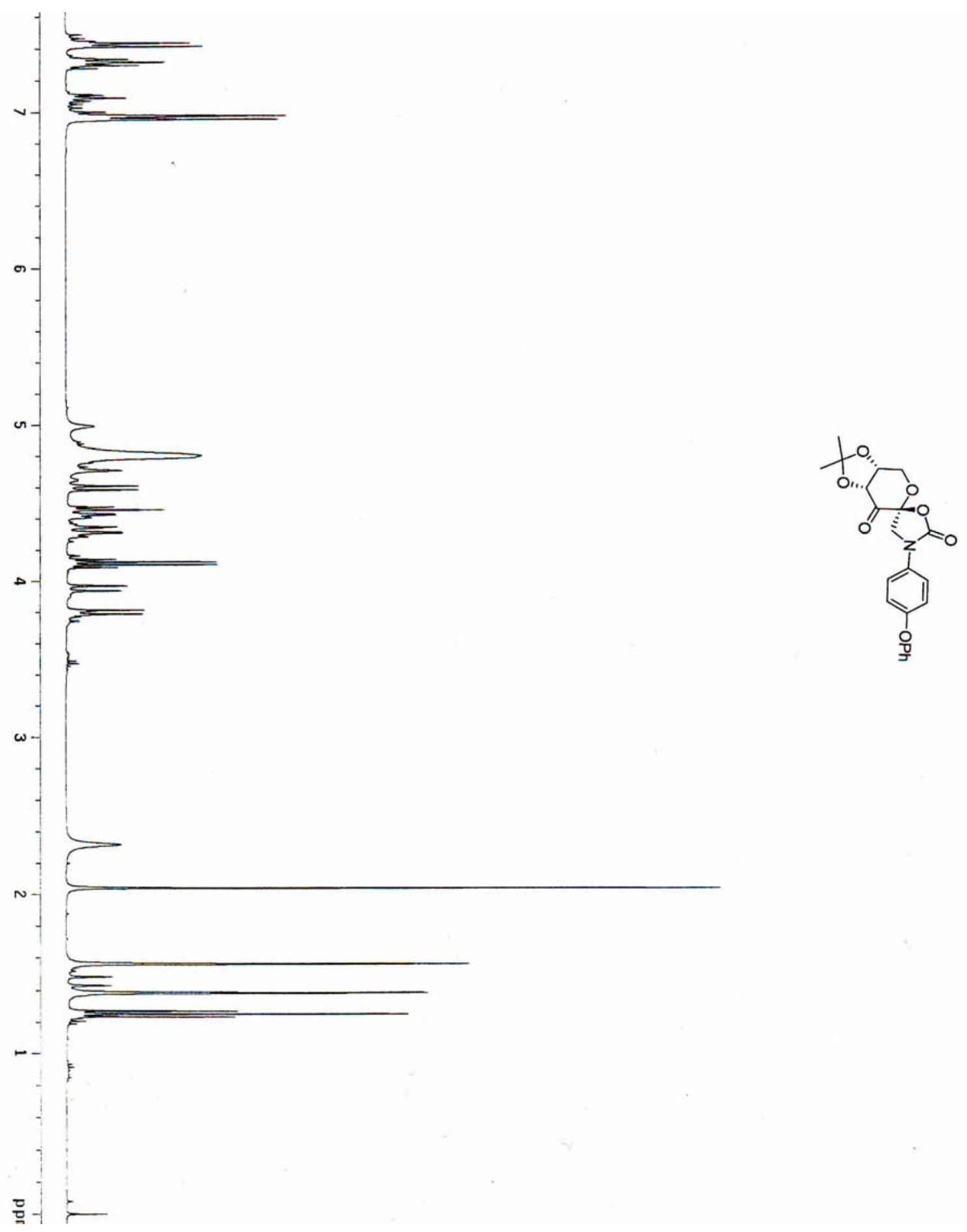




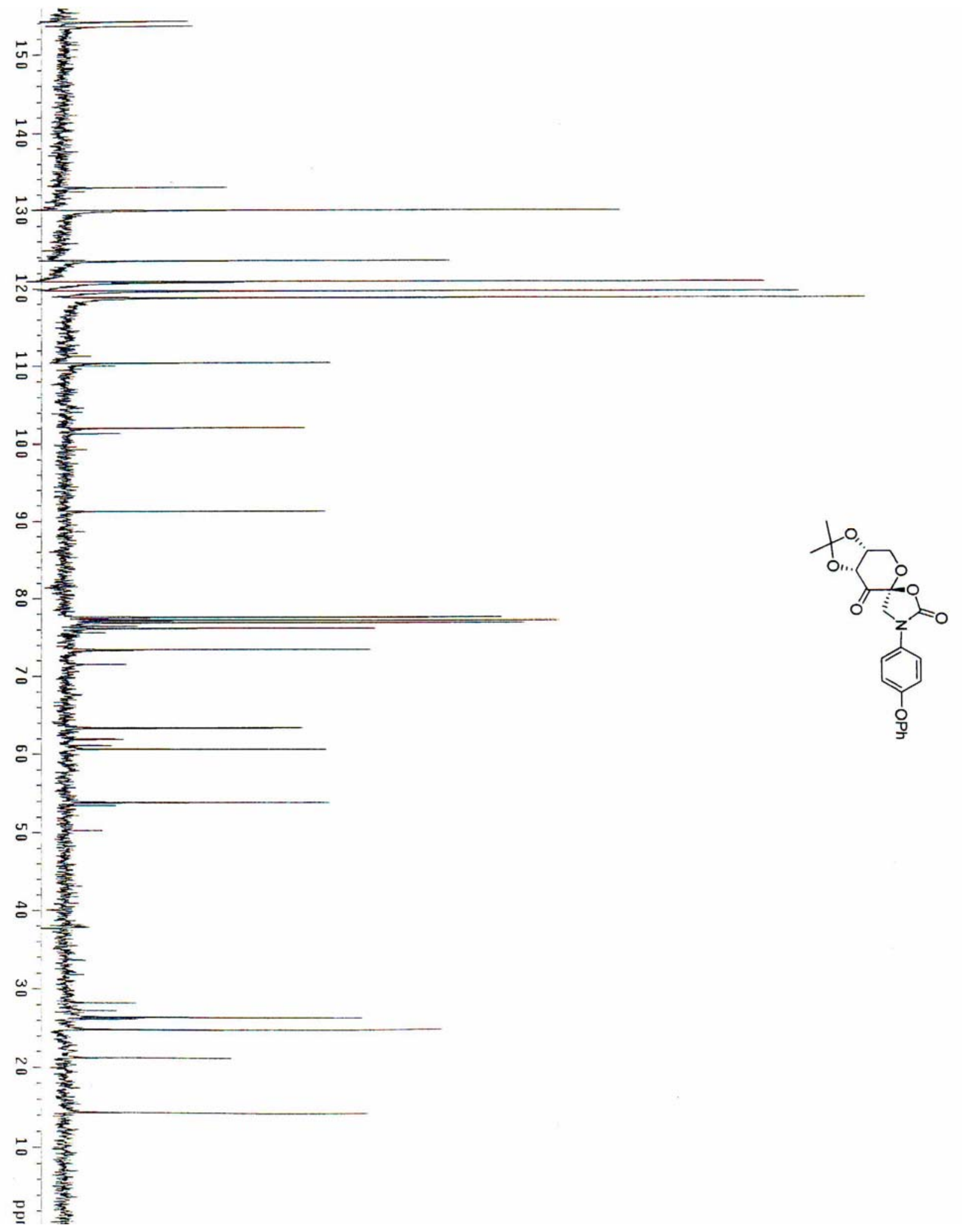



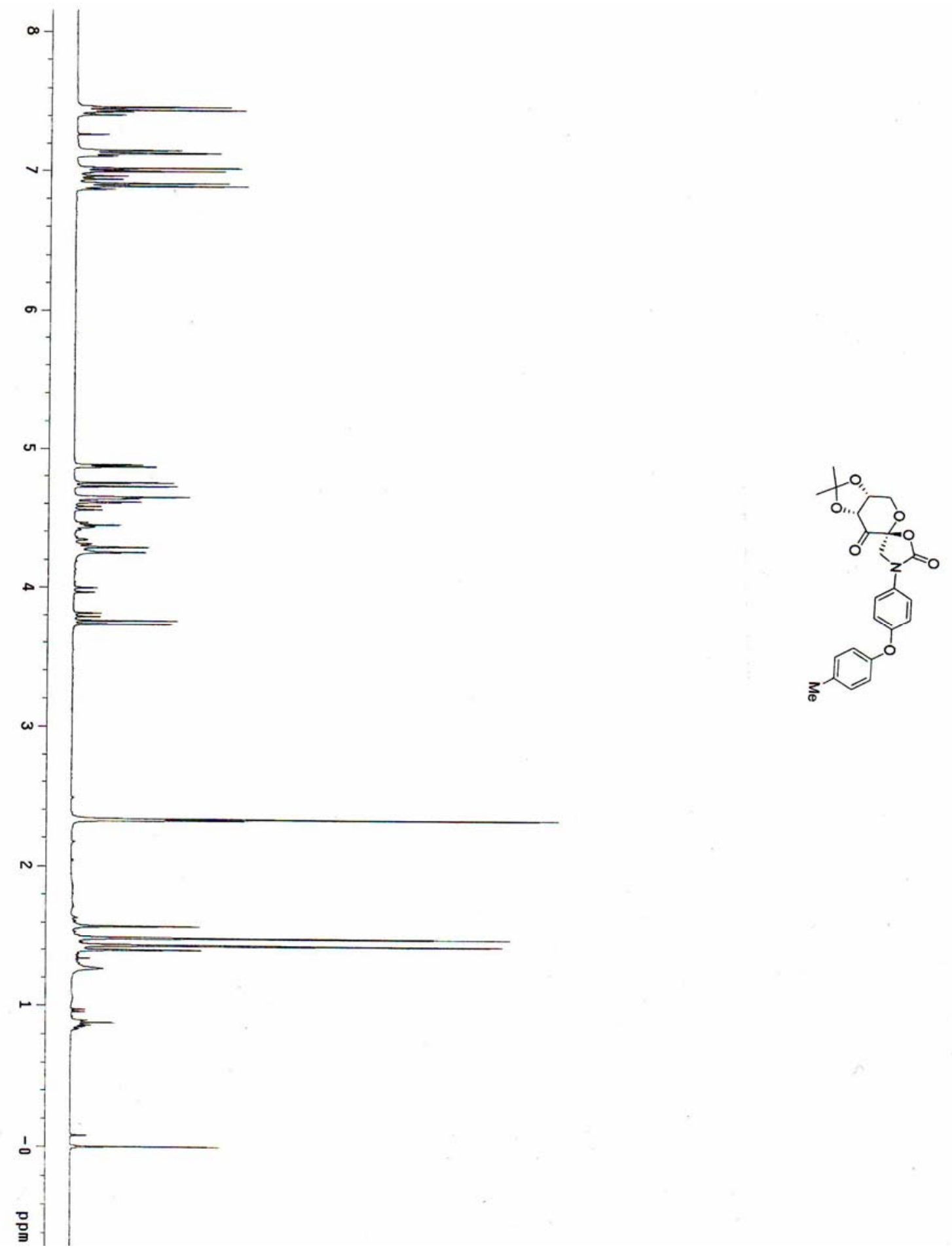


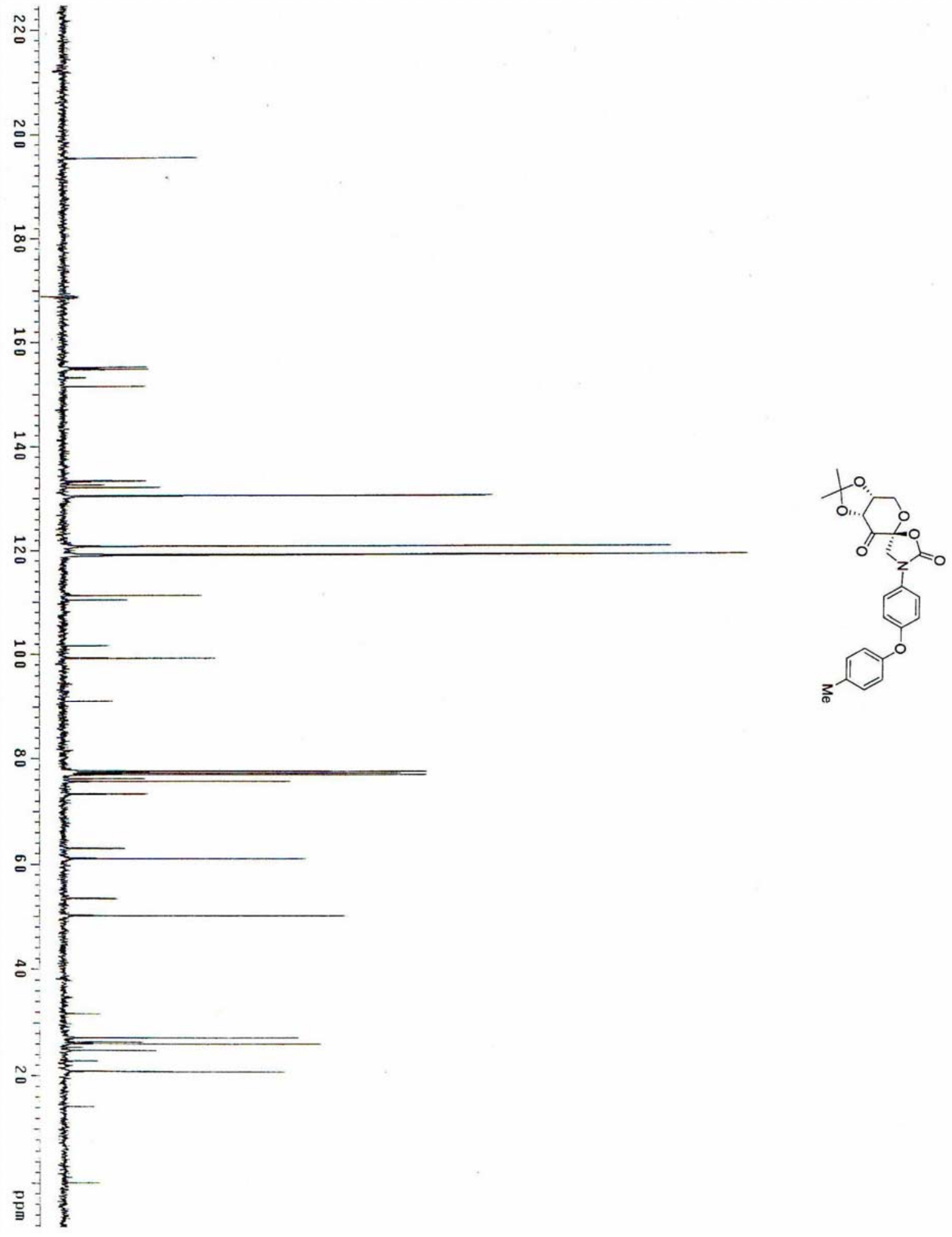



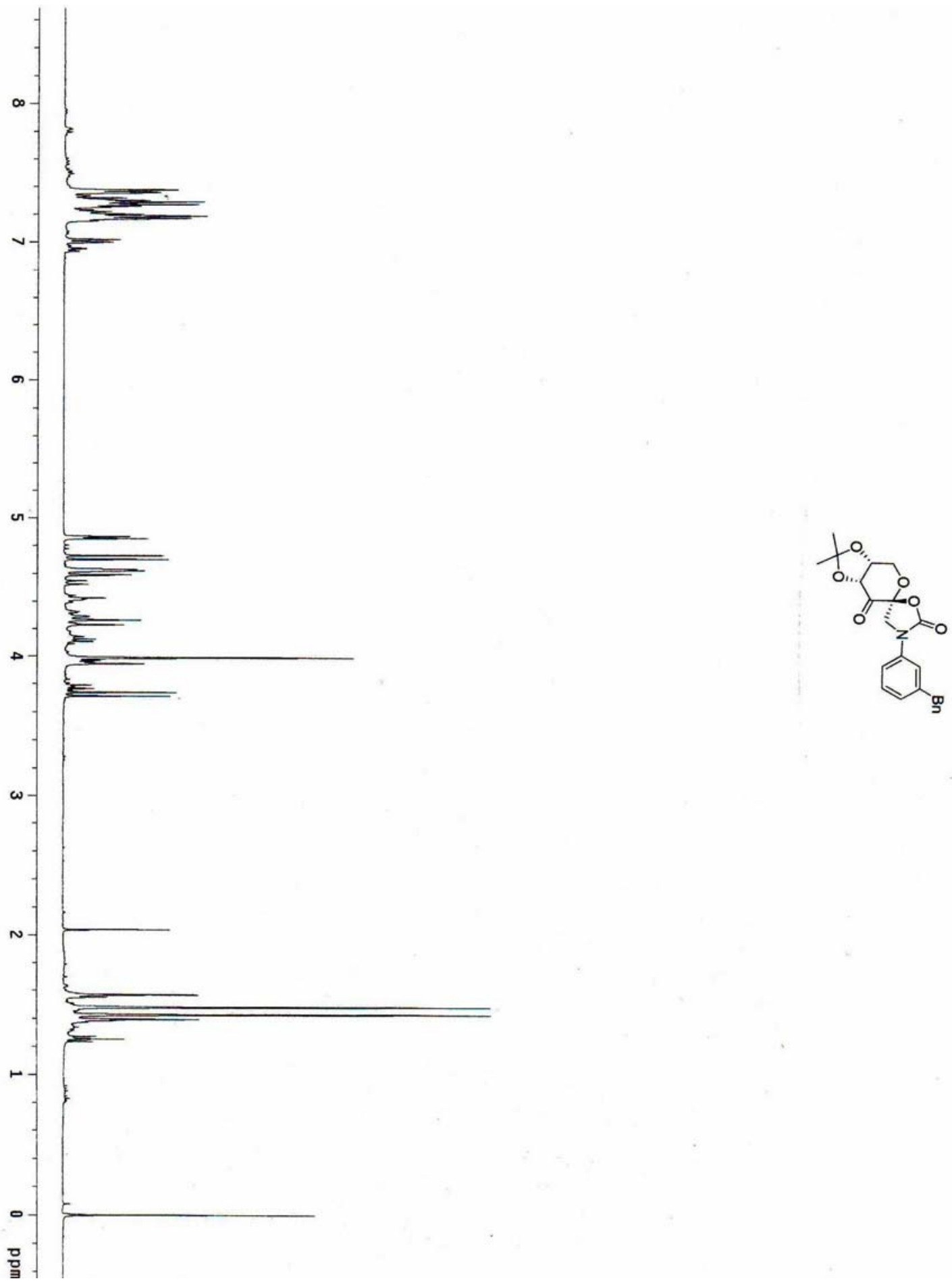


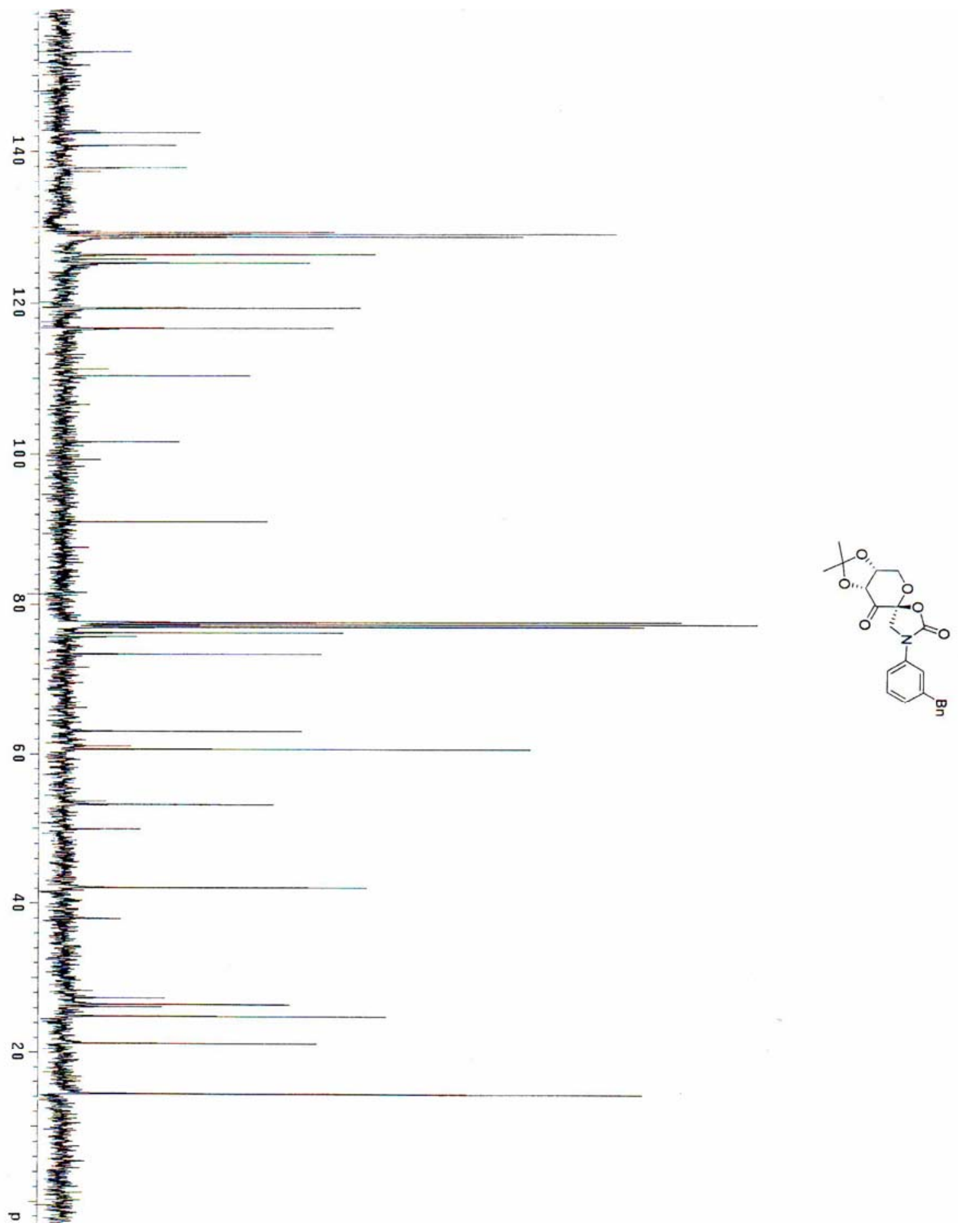



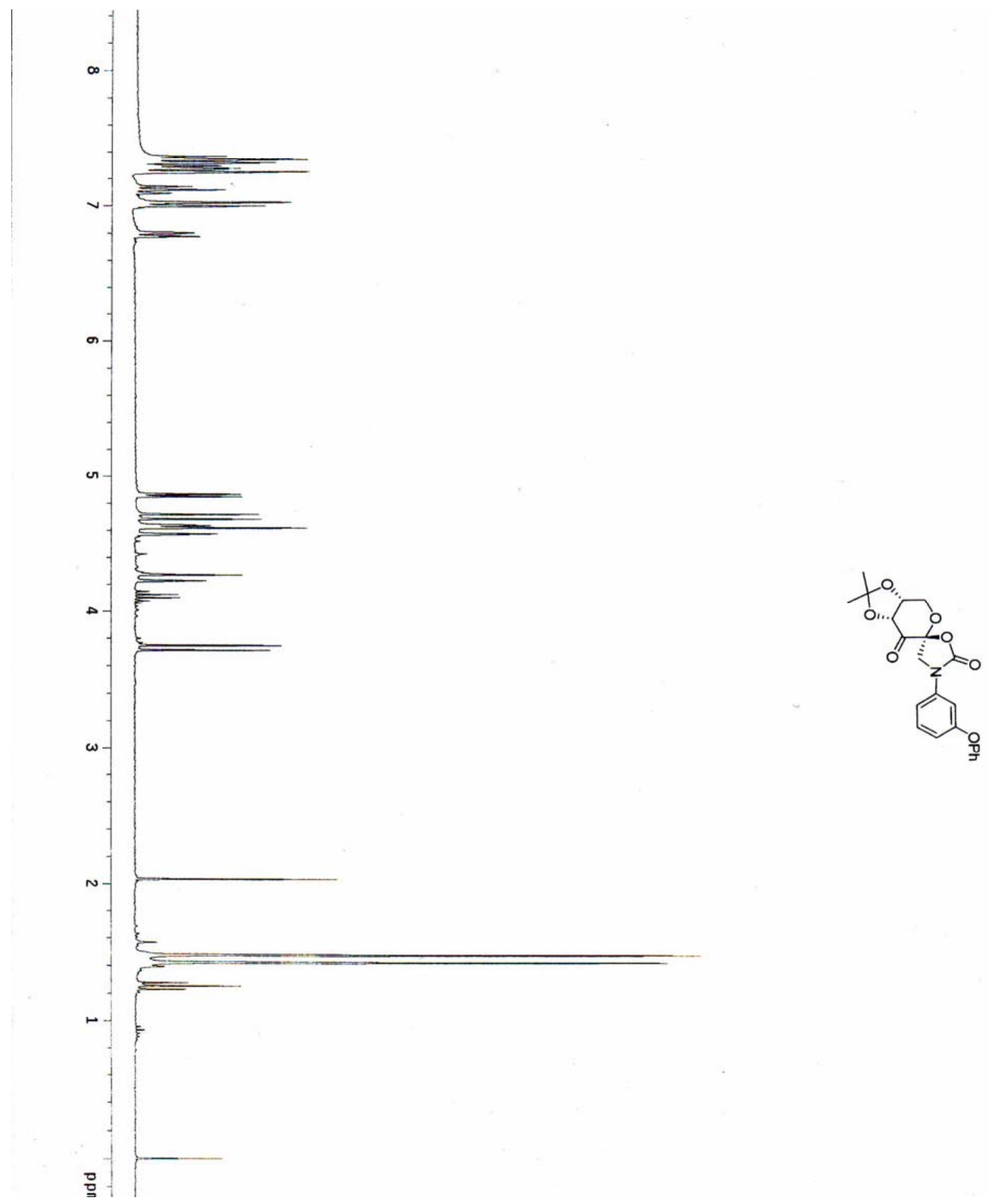

S41 


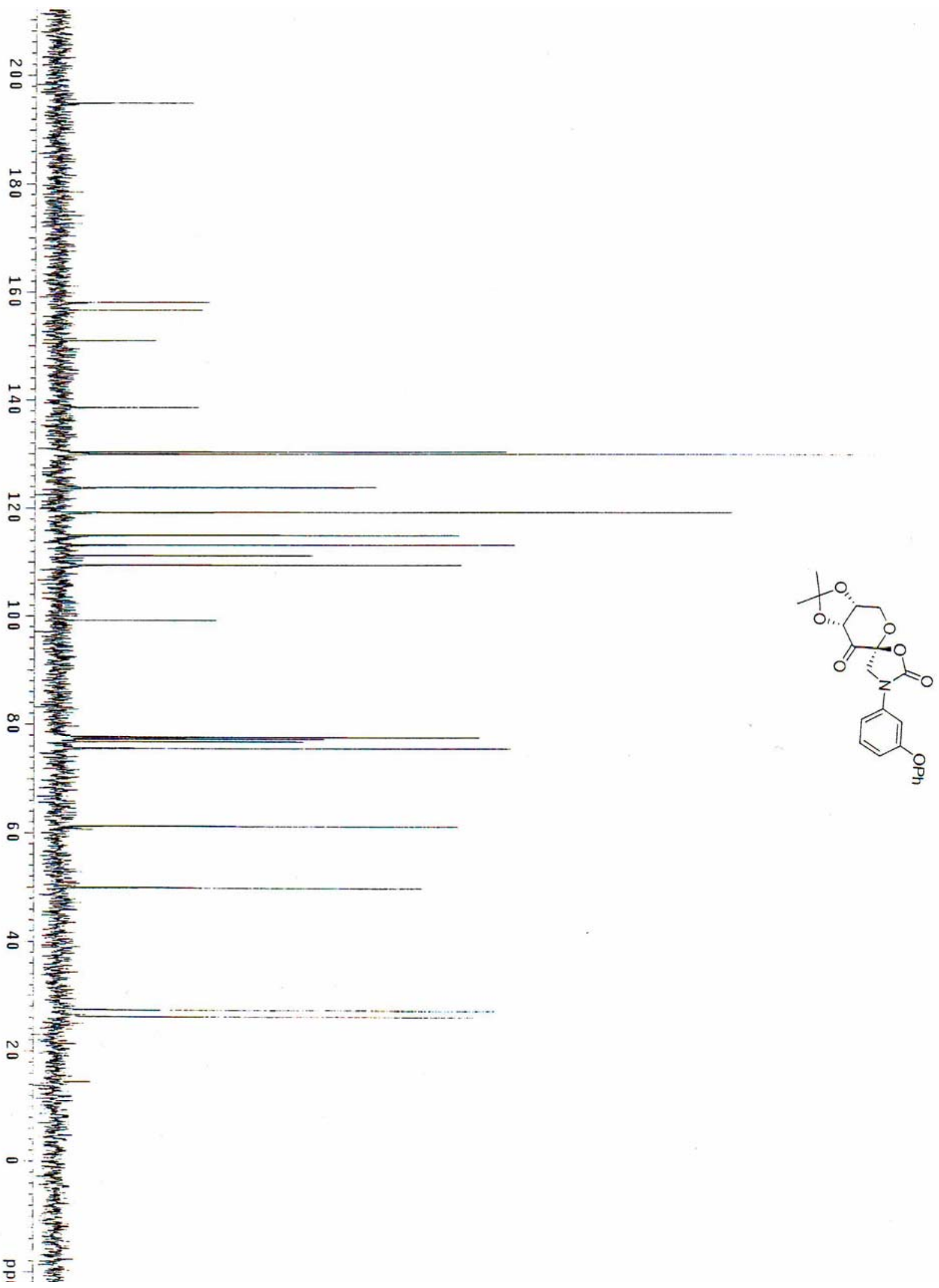




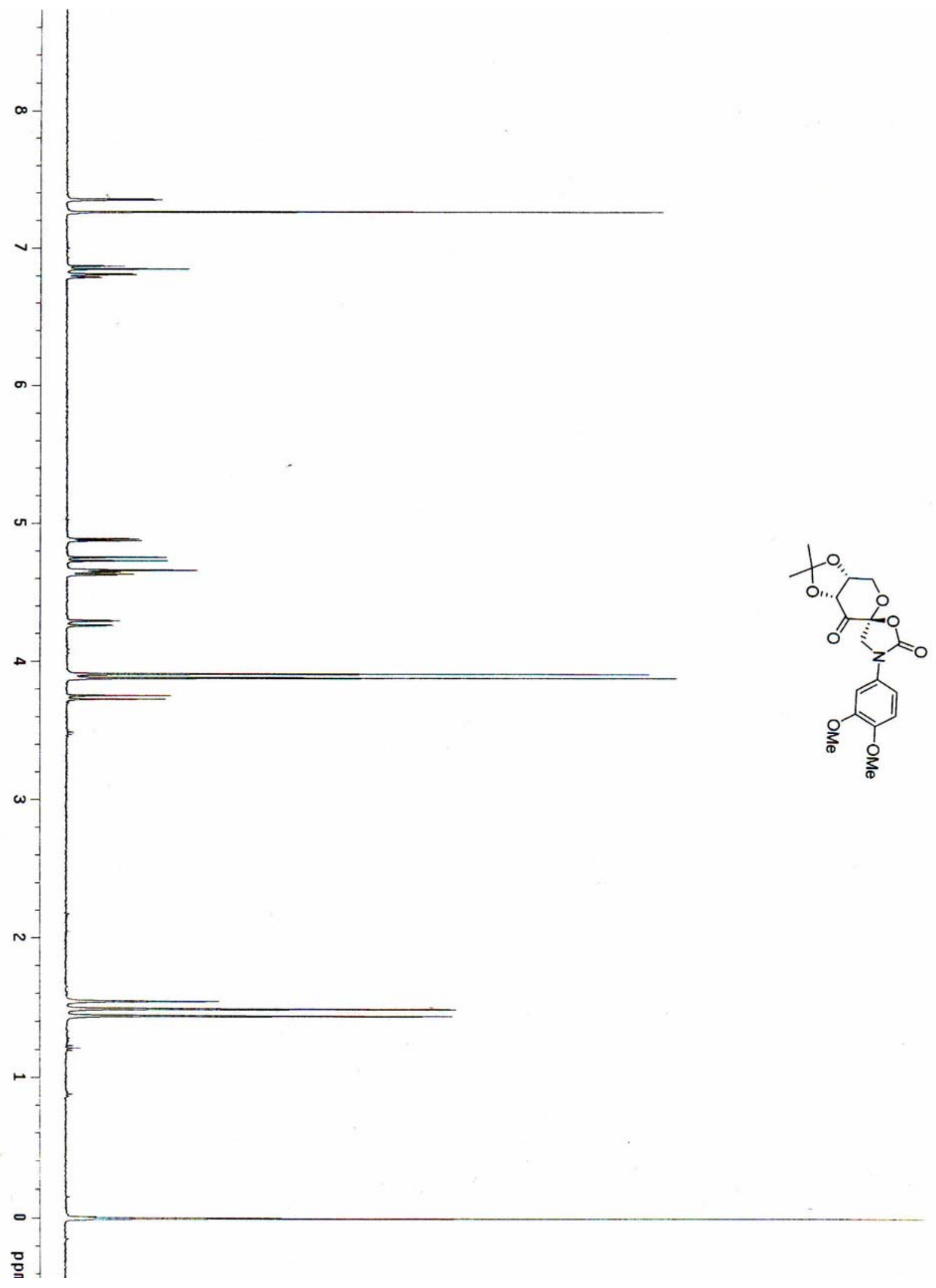




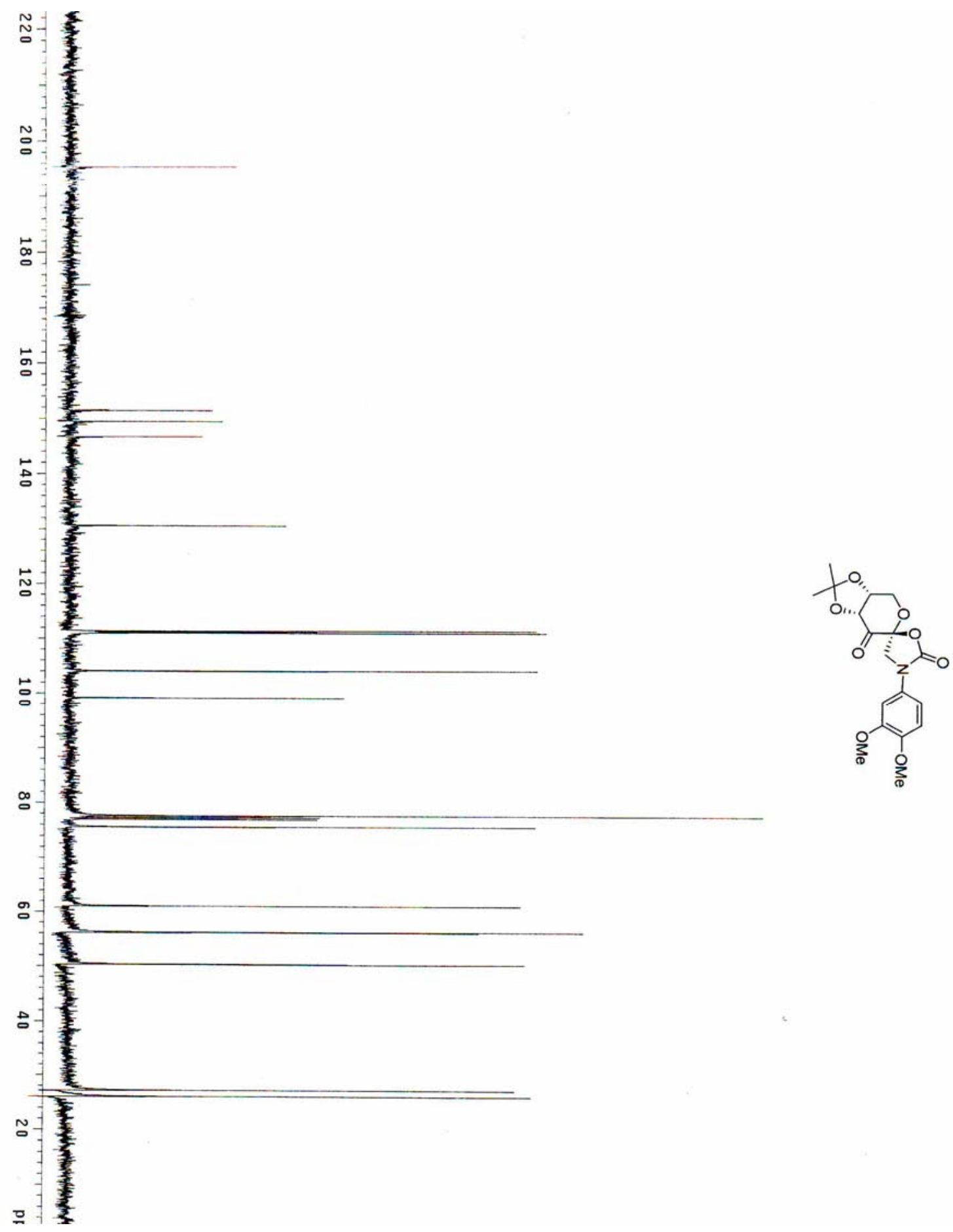




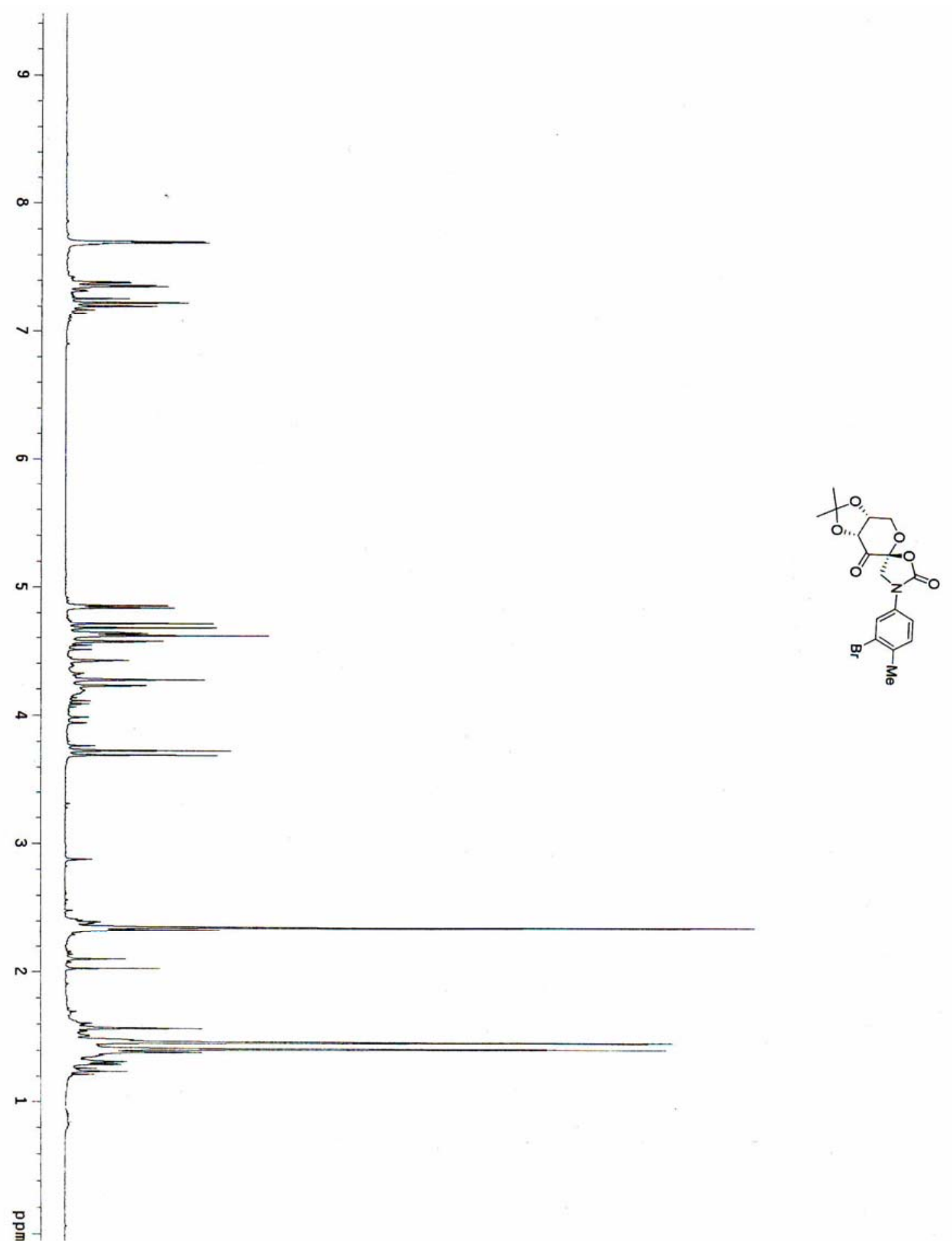




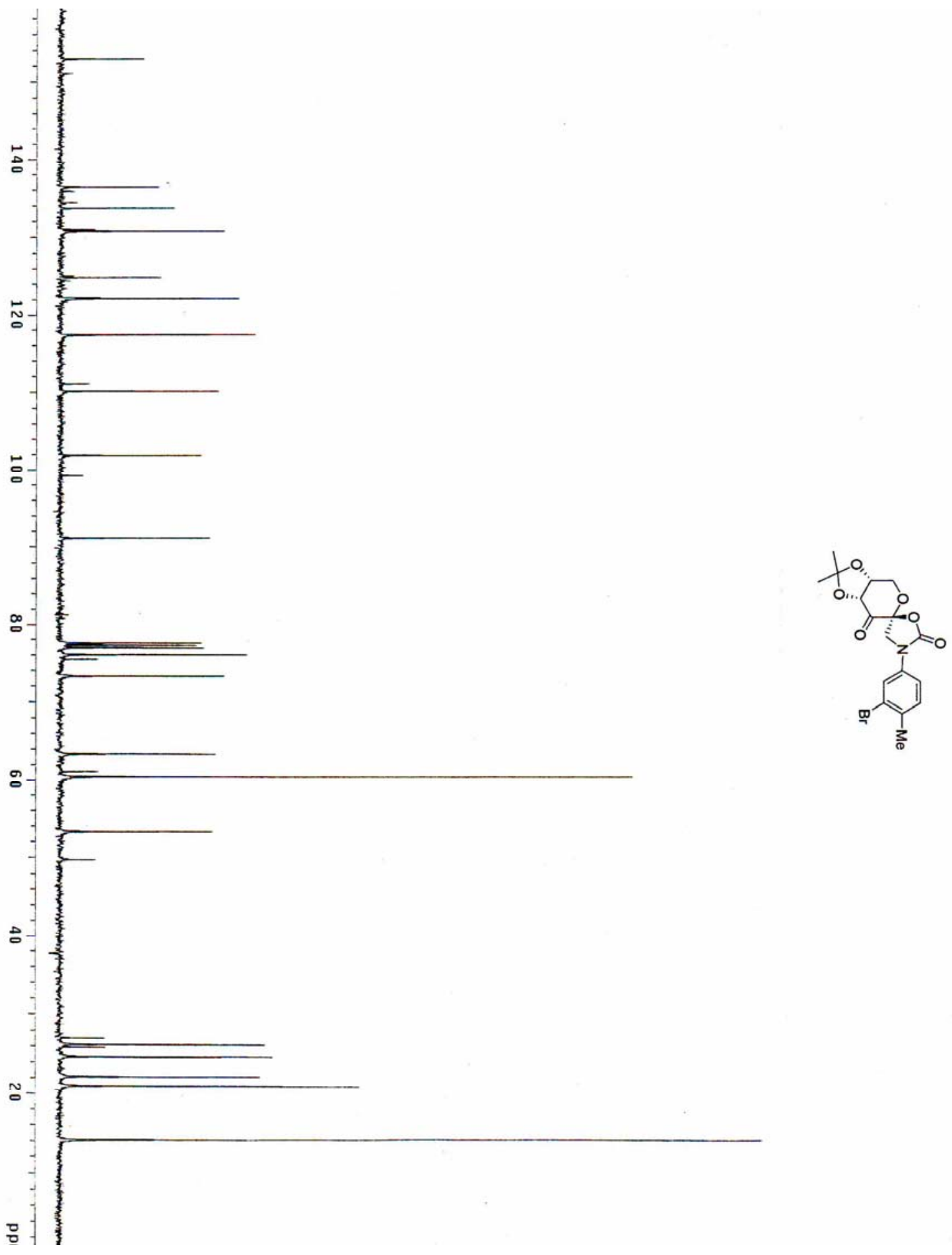



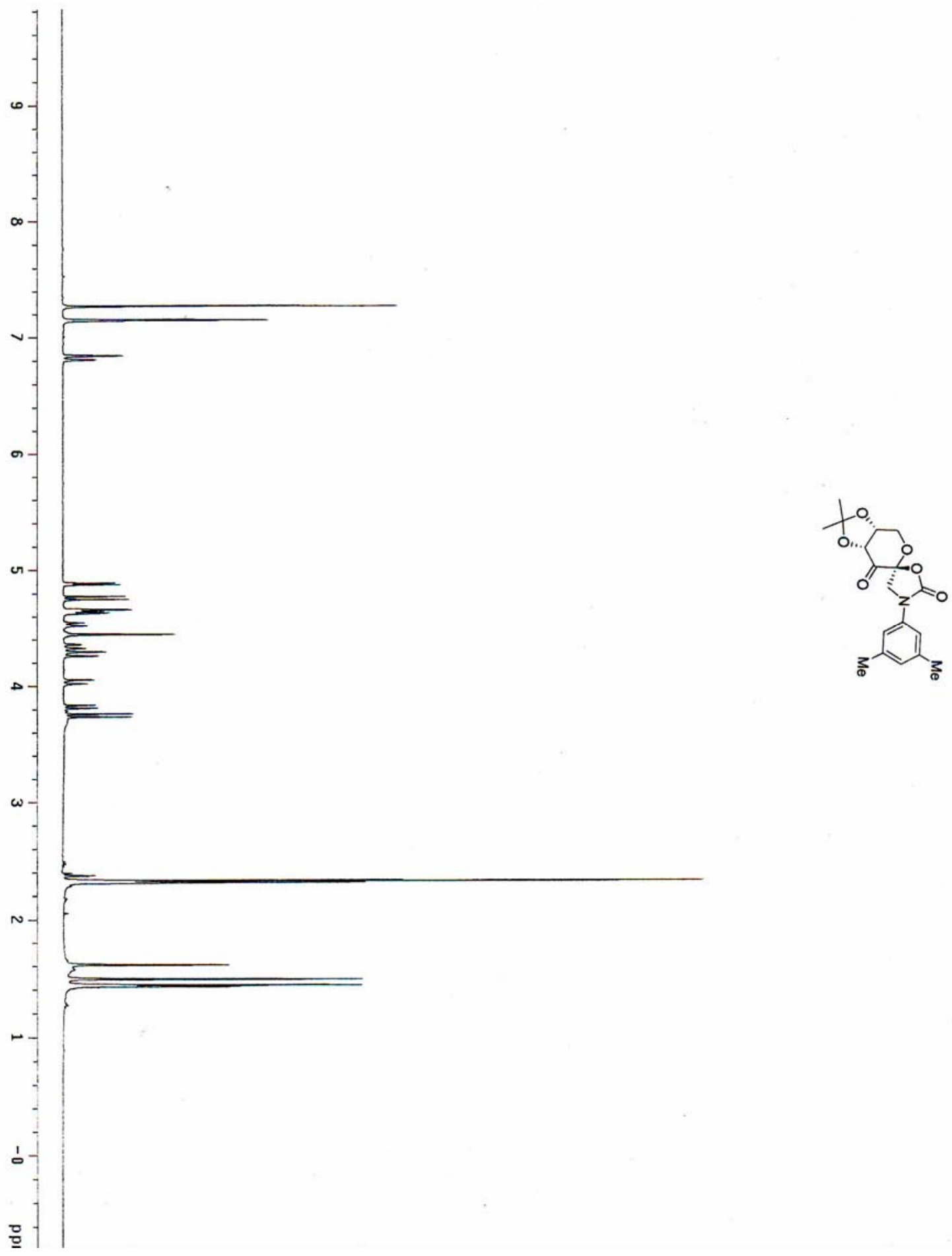


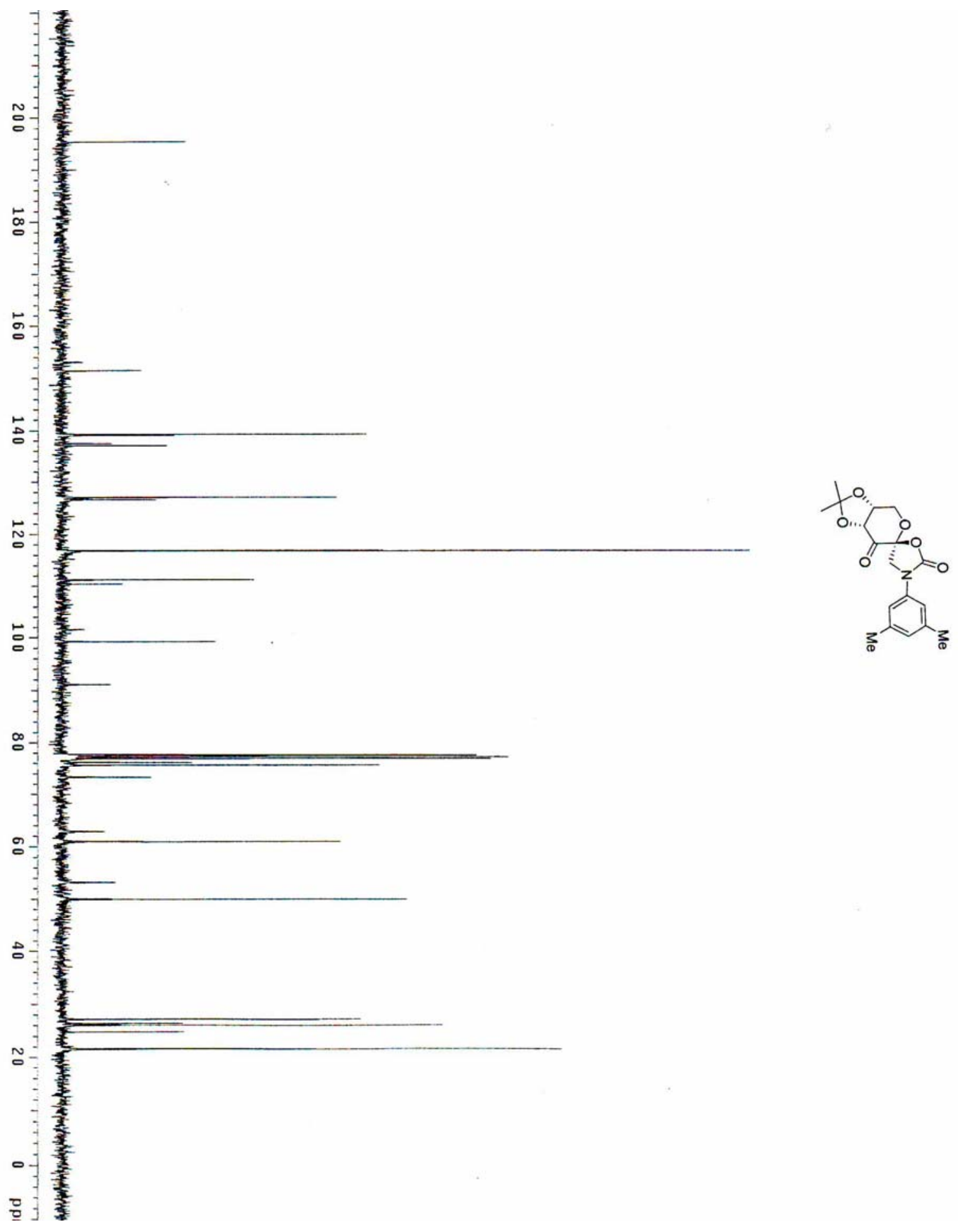




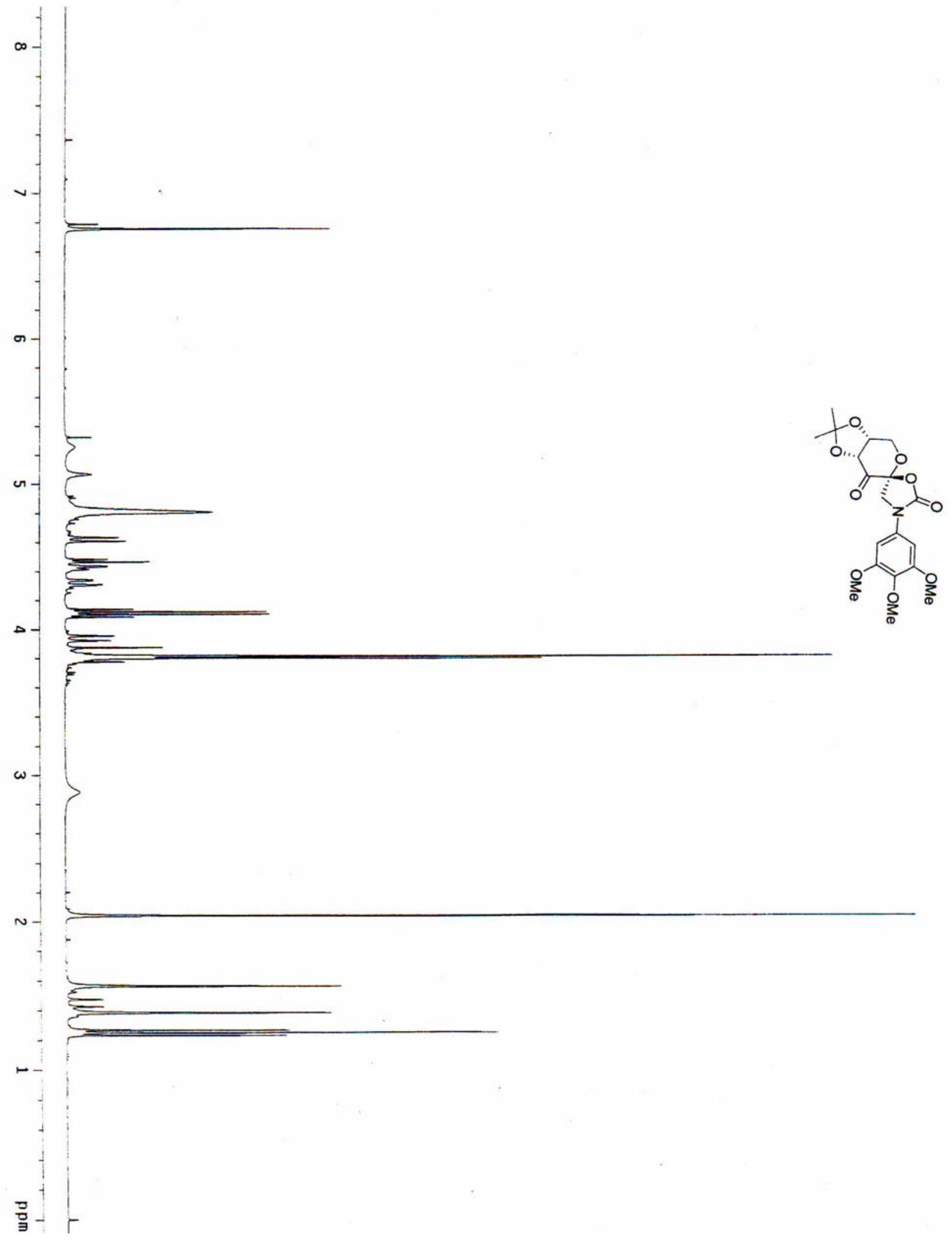



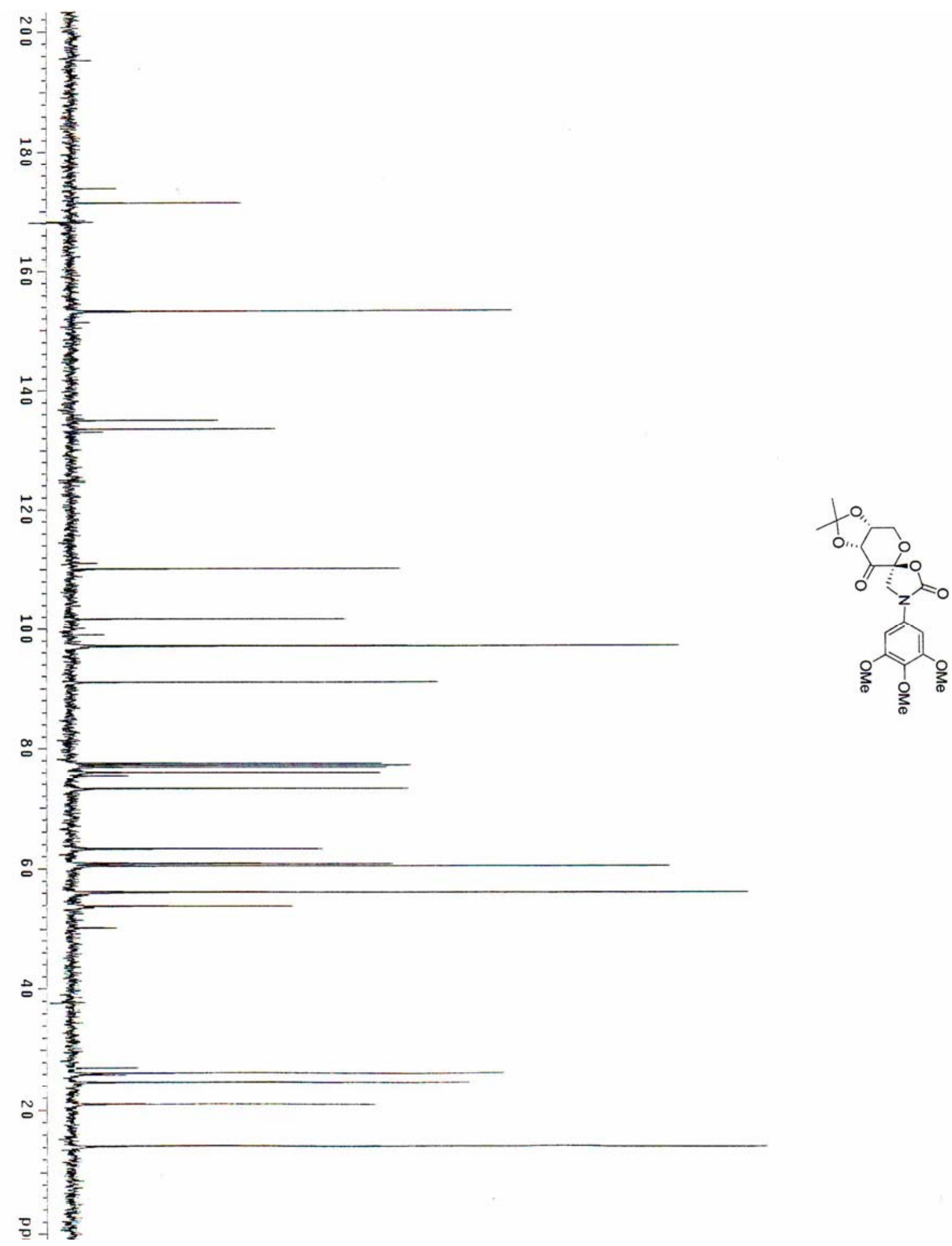


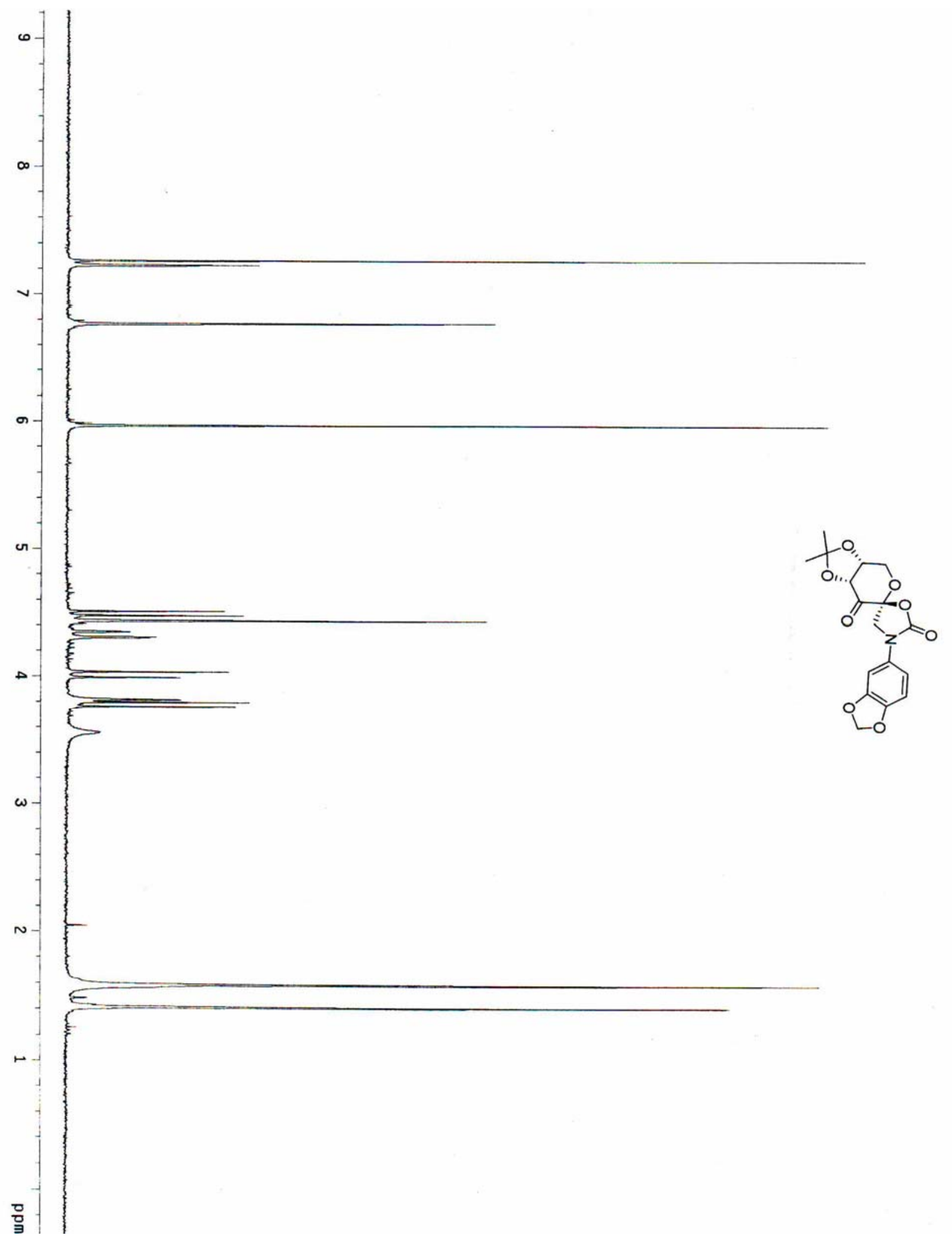




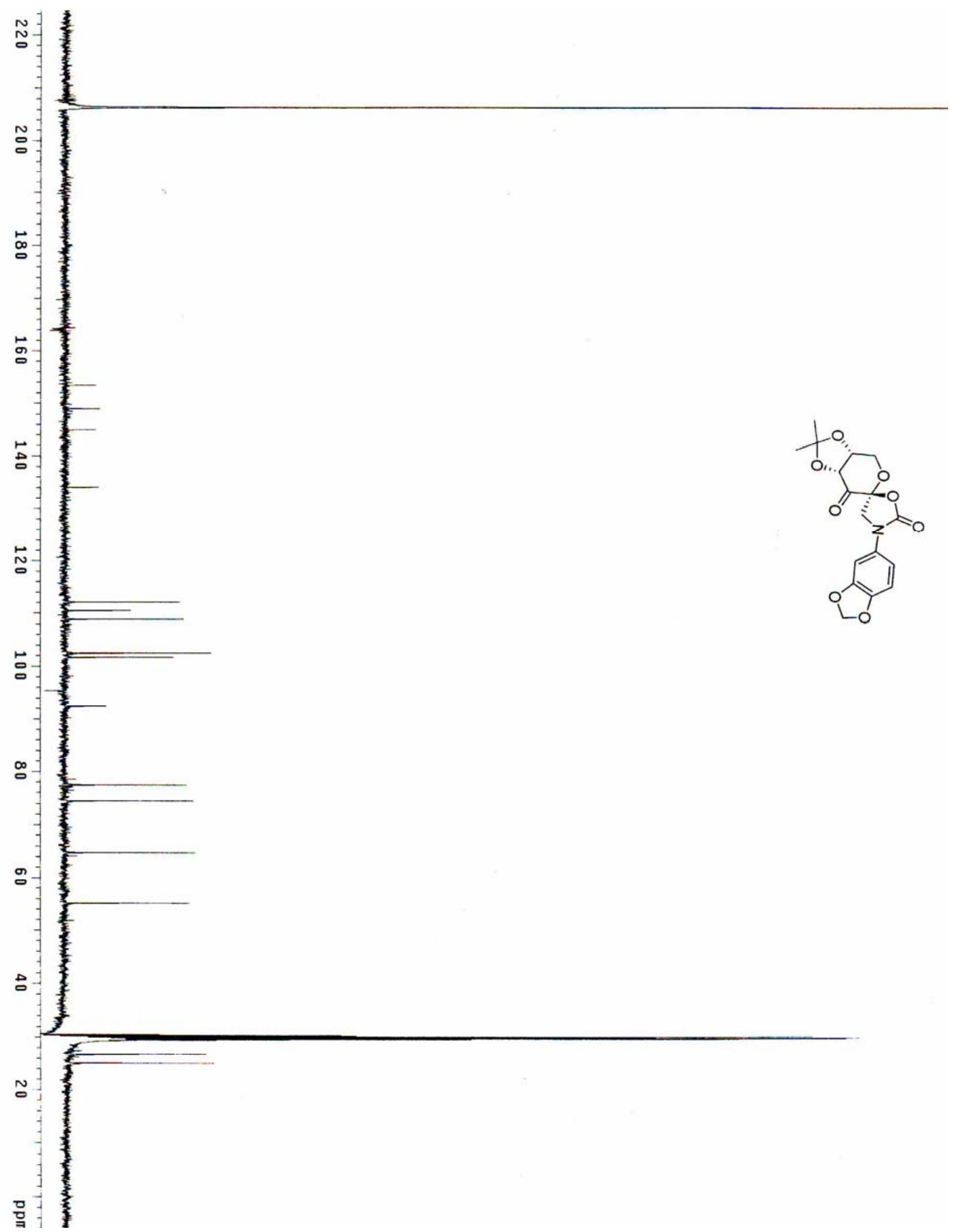




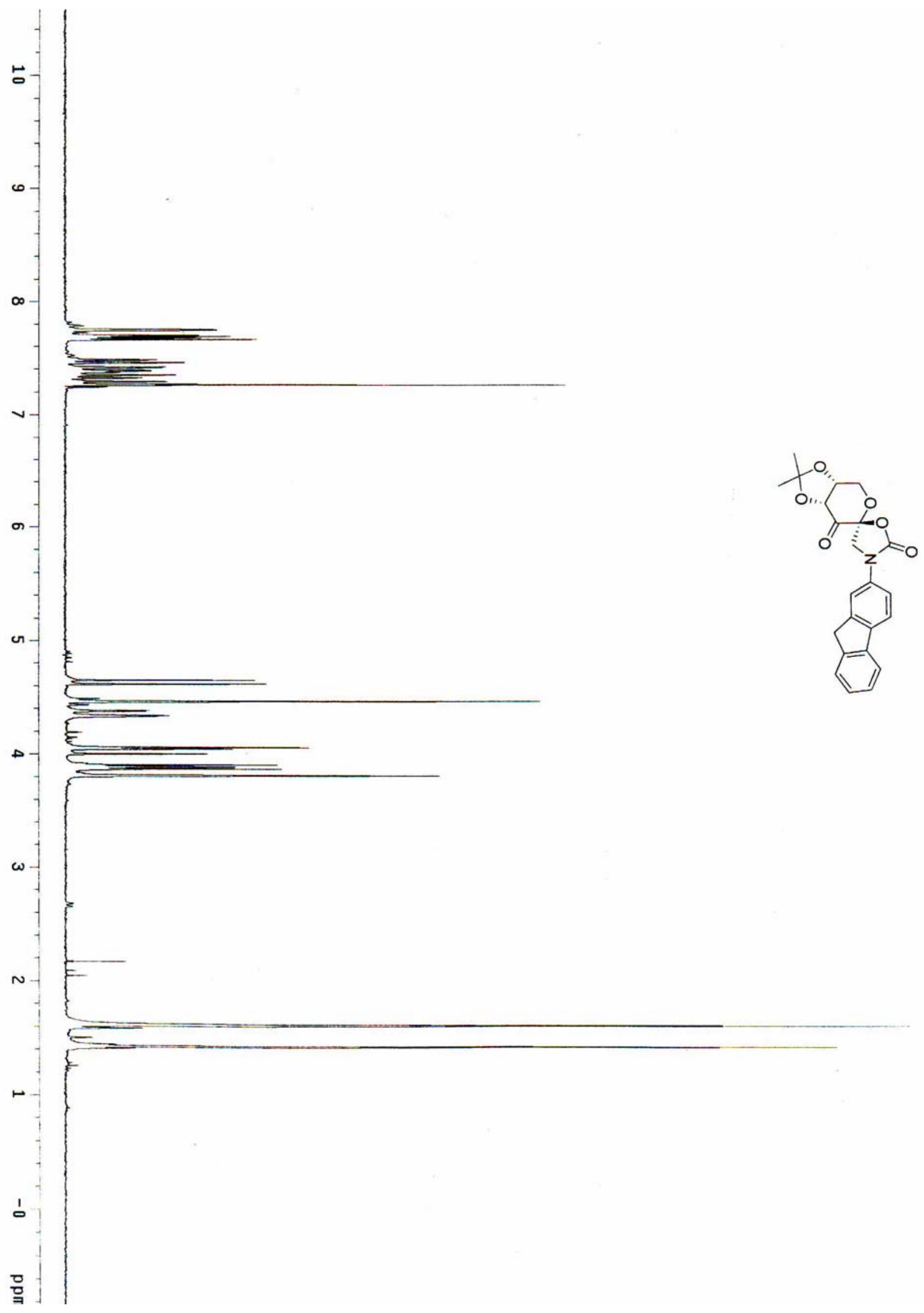




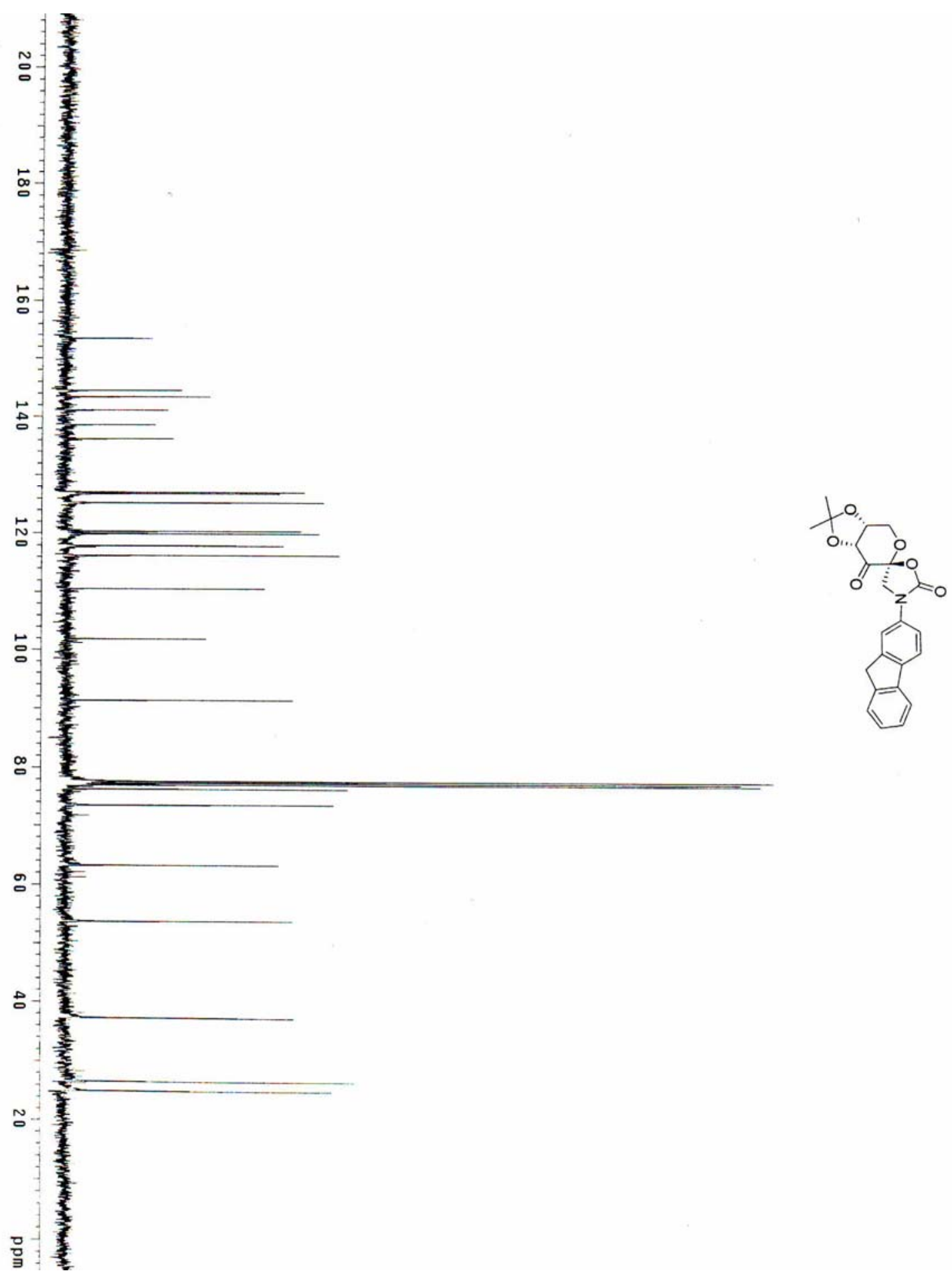

